\title{
A Novel Approach On Decision Support System Based On Triangular Linguistic Cubic Fuzzy Dombi Aggregation Operators
}

Muhammad Qiyas ( $\square$ muhammadqiyas@awkum.edu.pk)

Abdul Wali Khan University Mardan https://orcid.org/0000-0002-5519-6130

\section{Saleem Abdullah}

Abdul Wali Khan University Mardan

Ronnason Chinram

Prince of Songkla University

Muneeza .

Abdul Wali Khan University Mardan

\section{Research Article}

Keywords: Triangular linguistic cubic variable, Dombi operations, Triangular linguistic cubic variable Dombi averaging aggregation operators, Triangular linguistic intuitionistic cubic variable Dombi geometric aggregation operators

Posted Date: October 5th, 2021

DOl: https://doi.org/10.21203/rs.3.rs-905009/v1

License: (a) This work is licensed under a Creative Commons Attribution 4.0 International License. Read Full License

Version of Record: A version of this preprint was published at Soft Computing on January 8th, 2022. See the published version at https://doi.org/10.1007/s00500-021-06527-1. 


\title{
A NOVEL APPROACH ON DECISION SUPPORT SYSTEM BASED ON TRIANGULAR LINGUISTIC CUBIC FUZZY DOMBI AGGREGATION OPERATORS
}

\author{
MUHAMMAD QIYAS*, SALEEM ABDULLAH, RONNASON CHINRAM, AND MUNEEZA
}

\begin{abstract}
The triangular linguistic cubic fuzzy sets (TLCFSs) can express the fuzzy data easily, and also very useful in modeling of uncertain data in decision making (DM) problems. First of all, on the basis of Dombi t-norm and t-conorm (DTT), we propose novel operational rules of triangular linguistic cubic fuzzy numbers (TLCFNs). We propose some new aggregation operators of TLCFNs based on the newly-developed operations, i.e., triangular linguistic cubic fuzzy Dombi weighted averaging (TLCFDWA), triangular linguistic cubic fuzzy Dombi weighted geometric (TLCFDWG), triangular linguistic cubic fuzzy Dombi order weighted averaging (TLCFDOWA), triangular linguistic cubic fuzzy Dombi order weighted geometric (TLCFDOWG), triangular linguistic cubic fuzzy Dombi hybrid weighted averaging (TLCFDHWA), and triangular linguistic cubic fuzzy Dombi hybrid weighted geometric (TLCFDHWG) operators. Furthermore, a new method is proposed with the help of the proposed operators to solved the decision making problem. Finally, a numerical example is provided to illustrate the effectiveness of the new method. Comparative analysis is used to demonstrate the proposed method's superiority.
\end{abstract}

\section{INTRODUCTION}

Decision-making (DM) has played a key role in daily life, such as engineering, economics, medical, education, etc. In DM, one of the problems is the compilation of several information sources, giving the end result via aggregation process. Because of the complexities of management experience and decision-making issues, experts can give their ratings or decision to some degree, but may not be so sure of their judgments. In other words, there could be some degree of ambiguity which is a very important factor to consider when trying to develop very suitable strategies and solutions to the problems of decision. These ambiguities are seen effectively with intuitionistic fuzzy sets (IFSs) instead of precise numerical values. The definition of intuitionistic fuzzy sets (IFSs) [1] is the generalized type of Zadeh fuzzy sets (FSs) [56]. In IF set, the ordered pair denotes each number as positive grade and negative grade, and the sum of the positive grade and negative grade is less than or equal to one. Several researchers have made a valuable contribution to the improvement of the IF set and its applications, resulting in great success in the theoretical and technical aspects of IF sets.

Jun et al. [22] introduced cubic set, which are the theories of FS and IFS. Khan et al. [28] later on proposed aggregation operators for cubic sets. Mahmood et al.

\footnotetext{
Key words and phrases. Triangular linguistic cubic variable, Dombi operations, Triangular linguistic cubic variable Dombi averaging aggregation operators, Triangular linguistic intuitionistic cubic variable Dombi geometric aggregation operators.
} 
[35] presented cubic hesitant fuzzy sets and its application to MADM problems. Aliya et al. [11] proposed a new concept called triangular cubic fuzzy number, along with their operational laws, hamming distance and aggregating operators. Aliya et al. [12] developed the TCFHA operator and utilized it in WAR method to solve MADM problem. Aliya et al. [16] presented the gray relation analysis and cubic TOPSIS method. Shakeel [44] developed the arithmetic averaging operators, that are CWA operator, COWA operator and CHWA operator. Aliya et al. [17] developed TCFEHWA operators and applied them to MADM problems. Later on Amin et al. [2] proposed a new concept namely triangular cubic linguistic hesitant fuzzy sets (TCLHFSs) achieved by the generalization of TLHFS and TILHFS. And also established the TCLHFA operator, TCLHFG operator, GTCLHFWA operator, GTCHFWG operator, GTCLHFHG operator and GTCLHFHA operator. Aliya et al. [13] also developed three arithmetic averaging operators, CFEHWA operator, CFEWA operator and CFEOWA operator, to aggregate CF information. Aliya et al. [15] combined the Einstein operations and power average operator to create a cubic uncertain linguistic environment and proposed new operators, which are CULFPEA operator, CULFPEWA operator, CULFPEG operator and CULFPEWG operator. Amin et al. [3] proposed an approaches to MAGDM based on triangular cubic linguistic uncertain fuzzy aggregation operators. Senapati et al. $[26,45,46,47]$ applied cubic set theories in various algebraic structures. Muneeza et al. $[5,6]$ presented a new approach to intuitionistic cubic fuzzy set theory and applied the new structure in multicriteria group decision making. By using this concept, different problems arising in several areas can be solved by means of cubic sets as in the works of Rashid et al. [41], Ma et al. [36], Jun et al. [23, 24], Rashid et al. [42], Alhazaymeh et al. [4], and Fu et al. [14].

Zadeh [57], first time defined the linguistic variable that can estimate object evaluation. In many real-world cases, we still used linguistic terms like "excellent" and "bad" to describe a cricket player's success and can't convey few qualitative details with some numbers. Nevertheless, when linguistic variables are used to state qualitative information, this means that the degree of positive membership belongs to a language term and cannot be expressed in the degree of negative membership. To minimize this drawback, Wang and Li [48] implemented the intuitionistic linguistic set (ILS) by integrating the IFS with the linguistic variables. The example we described above can be given an "excellent" evaluation value for cricket player performance. This evaluation has an eighty percent degree of certainty, however, and the degree of negation is ten percent. Intuitionistic linguistic fuzzy collection can be used to guide the results of the assessment. This result can not, however, be conveyed through linguistic variables or intuitionistic fuzzy collection. In addition, [20,53] linguistic information allows us more space to evaluate ambiguous and imprecise information. Xu $[51,52]$ proposed linguistically weighted and ordered weighted average and geometric aggregation operators in the linguistic knowledge aggregation method. Chen et al. [8] applied linguistic intuitionist fuzzy sets (LIFSs) by taking positive and negative membership grades after their work and proposed their corresponding aggregation operators. Zhang [58] also described a number of LIFS aggregation operators. In the LIFS context, Peng et al. [37] defined Frank operations and Frank Heronian mean operator with application for assessing coal mines safety. Garg and Kumar [18] proposed a potential grade and average of LIFS operators. Wu and $\mathrm{Xu}$ [49] proposed an approach focused on distribution to 
solve MADM problems with unclear linguistic knowledge. Wang et al. [50] learned linguistic details about the MADM issue with IVIF. Xian et al. [54] proposed a problem-solving method under the linguistic picture fuzzy information, there are several methods for solving the MADM problem. Bordogna et al. [7] developed the linguistically ordered weighted average operators . Liao et al. [30] provided a model for selecting an ERP framework based on linguistic fuzzy knowledge. Multi-criteria linguistic decision-making model is proposed by Rodriguez et al. [39] in which experts provide their evaluations by obtaining description of languages. Herrera et al. [19] 2-tuple linguistic representation model to prevent the loss and misinterpretation of information during the processing of linguistic information. Martinez et al. [34] performed a survey of the 2-tuple linguistic model to rely on words when making decisions: extensions, implementations and challenges.

In 1982, Dombi [10] firstly defined Dombi norms, that have a great inclination of inconsistency with the operational parameters. Liu et al. [32] presented Dombi operations for IFS to solve MADM problems by establishing a Dombi Bonferroni mean operator. Chen and Ye [9] further generalized the Dombi operations to a single-valued neutrosophic set. He [21] developed Dombi hesitant fuzzy information aggregation operators and utilized it in Typhoon disaster assessment. Lu and Ye [33] proposed Dombi aggregation operators for the linguistic cubic variables (LCVs) and applied them to DM problems. Shi and Ye [43] also extended Dombi operations to NCSs and established MADM problem. Under Picture fuzzy knowledge, Jana et al. [27] provide Dombi aggregation operators with the DM methods to analyze the different preferences of the alternatives. Based on standard arithmetic, geometric and Dombi operations, Jana et al. [25] described some bipolar fuzzy Dombi aggregation operators. Recently, Ahmed et al. [29] proposed MADM problem utilizing Pythagorean Dombi averaging (PDA) operator and Pythagorean Dombi geometric (PDG) aggregation operator.

The model's key benefit is that it allows for the consideration of a wide range of situations depending on the decision maker's. It not only provides for the generalization of several helpful current aggregation operators, but also for the identification of some noteworthy operators and results. Motivated and inspired by the above discussion, in the present article we propose new operators using the Dombi norm and conorm for triangular linguistic cubic fuzzy variables (TCLFVs). The aggregation operators play an important role in aggregating the fuzzy data, during the DM process. In this study, we proposed some averaging and geometric aggregation operators for the TLCFVs using Domi operation laws, such as; triangular linguistic cubic fuzzy Dombi weighted average (TLCFDWA), triangular linguistic cubic fuzzy Dombi order weighted average (TLCFDOWA), triangular linguistic cubic fuzzy Dombi hybrid weighted average (TLCFDHA), triangular linguistic cubic fuzzy Dombi weighted geometric (TLCFDWG), triangular linguistic cubic fuzzy Dombi order weighted geometric (TLCFDOWG) and triangular linguistic cubic fuzzy Dombi hybrid weighted geometric (TLCFDHG) operators. After that, we use the proposed Dombi operators to construct a triangular linguistic cubic fuzzy MADM problem. A numerical illustration shows how efficient our proposed approach is, and helps us to use triangular linguistic cubic fuzzy data in DM problem.

The remaining article is arranged as follows: In Section 2, we briefly introduce basic knowledge about the linguistic term set, linguistic fuzzy set (LFS), linguistic 
cubic variable and triangular linguistic cubic fuzzy variable. In Section 3, we proposed new operation laws for triangular linguistic cubic fuzzy variables (TLCFVs) using Dombi t-norm and conorm. The aggregation operators and the properties of TLCFVs are discussed in Section 4. In Section 5, an approach for MADM problem are discussed. A numerical application is presented in Section 6. Finally, we discussed the benefits and conclusions of the proposed study in Section 7 .

\section{Preliminaries}

Some basic concepts and knowledge regarding triangular number, linguistic cubic variable and Dombi operation are briefly described in the following.

Definition 1. [19] Let $S=\left(s_{1}, \ldots, s_{t}\right)$ are the finite order distinct term set. The term $S$ is called the linguistic term set, and $t$ is called the odd cardinality, e.g., $3,5, \ldots$, when $t=5$, then $\dot{S}$ can be defined as $\dot{S}=\left(s_{1}, s_{2}, s_{3}, s_{4}, s_{5}\right)=$ (poor, slightly poor, fair, slightly good, good)

The linguistic term set $S$ satisfies the following properties;

(1) Ordered : $s_{i} \prec s_{l} \Leftrightarrow i \prec l$

(2) Negation: neg $\left(s_{i}\right)=s_{t-1-i}$

(3) Maximum: $\left(s_{i}, s_{l}\right)=s_{i}$, iff $i \geq l$

(4) Minimum: $\left(s_{i}, s_{l}\right)=s_{i}$, iff $i \leq l$.

Continues linguistic term set (CLTS) is the extended form of a discrete term set $\dot{S}$ and defined as $\dot{S}^{*}=\left\{s_{\psi} \mid s_{0} \leq s_{\psi} \leq s_{g}, \psi \in[0, t]\right.$, if $s_{\psi} \in \dot{S}^{*}$ then $s_{\psi}$ is known as original term otherwise, the virtual term.

Definition 2. [57] Let $\mathbb{R} \neq \phi$ be a set. A linguistic fuzzy set $\Re$ in $\mathbb{R}$ is described as follows;

$$
\Re=\left\{\left(r, s_{x_{\Re}}(r)\right) \mid r \in \mathbb{R}\right\},
$$

where $s_{x_{\Re}}: \mathbb{R} \rightarrow[0, t]$ is the linguistic membership grade of $\Re$.

Definition 3. [55] A linguistic cubic variables $\Re$ in $\mathbb{R} \neq \phi$ is given as follows;

$$
\Re=\left\{\left(r,\left[s_{x_{\Re}^{-}}(r), s_{x_{\Re}^{+}}(r)\right] ; s_{z_{\Re}}(r)\right) \mid r \in \mathbb{R}\right\},
$$

where the first element in linguistic cubic variables denotes the grade of linguistic membership and second is a simple linguistic fuzzy number.

Definition 4. Let $S^{*}=\left\{s_{\psi} \mid s_{0} \leq s_{\psi} \leq s_{g}, \psi \in[0, t]\right.$, be a continues linguistic term set. Then, a triangular linguistic cubic fuzzy variable (TLCFV) is defined as;

$$
\Re=\left\{\left\langle\left[s_{x^{-}}, s_{y^{-}}, s_{z^{-}}\right],\left[s_{x^{+}}, s_{y^{+}}, s_{z^{+}}\right]\right\rangle ; s_{\varkappa}, s_{\phi}, s_{\varphi}\right\} .
$$

Where the first element in triangular linguistic cubic fuzzy variables denotes the grade of triangular linguistic membership and second is a simple triangular linguistic fuzzy number. If $x^{-}+y^{-}+z^{-} \leq \varkappa+\phi+\varphi \leq x^{+}+y^{+}+z^{+}$, then $\Re=$ $\left\{\left\langle\left[s_{x^{-}}, s_{y^{-}}, s_{z^{-}}\right],\left[s_{x^{+}}, s_{y^{+}}, s_{z^{+}}\right]\right\rangle ; s_{\varkappa}, s_{\phi}, s_{\varphi}\right\}$ is an internal TLCFVs, and if $\varkappa+\phi+$ $\varphi<x^{-}+y^{-}+z^{-}$or $\varkappa+\phi+\varphi>x^{+}+y^{+}+z^{+}$, then $\Re=\left\{\left\langle\left[s_{x^{-}}, s_{y^{-}}, s_{z^{-}}\right],\left[s_{x^{+}}, s_{y^{+}}, s_{z^{+}}\right]\right\rangle ; s_{\varkappa}, s_{\phi}, s_{\varphi}\right\}$ is an external TLCFVs.

Definition 5. Let $\Re=\left\{\left\langle\left[s_{x^{-}}, s_{y^{-}}, s_{z^{-}}\right],\left[s_{x^{+}}, s_{y^{+}}, s_{z^{+}}\right]\right\rangle ; s_{\varkappa}, s_{\phi}, s_{\varphi}\right\}$ be a triangular linguistic cubic fuzzy variable. Then, the score function is defined as;

$$
S c(\Re)=\frac{x^{-}+y^{-}+z^{-}+x^{+}+y^{+}+z^{+}+\varkappa-\phi-\varphi}{9 t}, S c(\Re) \in[-1,1] .
$$


Definition 6. Let $\Re_{1}=\left\{\left\langle\left[s_{x_{1}^{-}}, s_{y_{1}^{-}}, s_{z_{1}^{-}}\right],\left[s_{x_{1}^{+}}, s_{y_{1}^{+}}, s_{z_{1}^{+}}\right]\right\rangle ; s_{\varkappa_{1}}, s_{\phi_{1}}, s_{\varphi_{1}}\right\}$ and $\Re_{2}=\left\{\left\langle\left[s_{x_{2}^{-}}, s_{y_{2}^{-}}, s_{z_{2}^{-}}\right],\left[s_{x_{2}^{+}}, s_{y_{2}^{+}}, s_{z_{2}^{+}}\right]\right\rangle ; s_{\varkappa_{2}}, s_{\phi_{2}}, s_{\varphi_{2}}\right\}$ are the two triangular linguistic cubic fuzzy variables. Then, their score value comparison rules are defined as;

- If $S c\left(\Re_{1}\right)>S c\left(\Re_{2}\right)$, then $\Re_{1}>\Re_{2}$;

- If $S c\left(\Re_{1}\right)<S c\left(\Re_{2}\right)$, then $\Re_{1}<\Re_{2}$;

- If $S c\left(\Re_{1}\right)=S c\left(\Re_{2}\right)$, then $\Re_{1}=\Re_{2}$.

\section{Dombi Operation for Triangular Linguistic Cubic Fuzzy Variables}

Definition 7. [10] Let we have two real numbers $\Delta$ and $\Lambda$. If $(\Delta, \Lambda) \in[0,1] \times[0,1]$. Then, Dombi t-norm and t-conorm is defined as;

$$
\begin{aligned}
\operatorname{Dom}(\Delta, \Lambda) & =\frac{1}{1+\left\{\left(\frac{1-\Delta}{\Delta}\right)^{\rho}+\left(\frac{1-\Lambda}{\Lambda}\right)^{\rho}\right\}^{\frac{1}{\rho}}}, \\
\operatorname{Dom}^{c}(\Delta, \Lambda) & =\frac{1}{1+\left\{\left(\frac{\Delta}{1-\Delta}\right)^{\rho}+\left(\frac{\Lambda}{1-\Lambda}\right)^{\rho}\right\}^{\frac{1}{\rho}}}
\end{aligned}
$$

where, $\rho>0, \operatorname{Dom}(\Delta, \Lambda) \in[0,1]$ and $\operatorname{Dom}^{c}(\Delta, \Lambda) \in[0,1]$.

Definition 8. Consider two triangular linguistic cubic fuzzy variables $\Re_{1}=\left\{\left\langle\left[s_{x_{1}^{-}}, s_{y_{1}^{-}}, s_{z_{1}^{-}}\right],\left[s_{x_{1}^{+}}, s_{y_{1}^{+}}, s_{z_{1}^{+}}\right]\right\rangle ; s_{\varkappa_{1}}\right.$ and

$\Re_{2}=\left\{\left\langle\left[s_{x_{2}^{-}}, s_{y_{2}^{-}}, s_{z_{2}^{-}}\right],\left[s_{x_{2}^{+}}, s_{y_{2}^{+}}, s_{z_{2}^{+}}\right]\right\rangle ; s_{\varkappa_{2}}, s_{\phi_{2}}, s_{\varphi_{2}}\right\}$, with $\rho \geq 1, \lambda>0$. Then, the operation laws of triangular linguistic cubic fuzzy variables based on Dombi operations define as follows; 
MUHAMMAD QIYAS*, SALEEM ABDULLAH, RONNASON CHINRAM, AND MUNEEZA

(2)

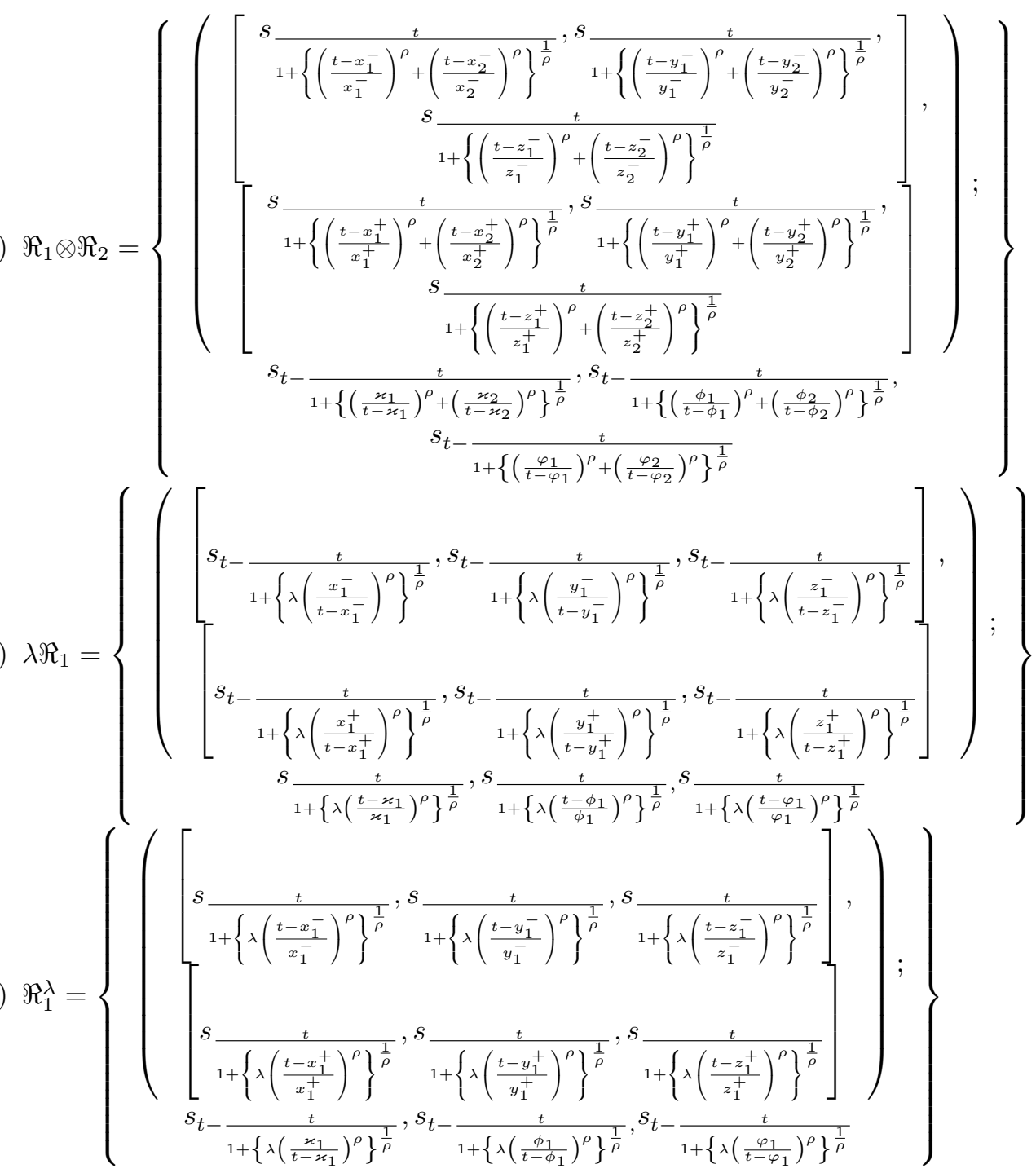

Theorem 1. Let three triangular linguistic cubic fuzzy variables $\Re_{1}=\left\{\left\langle\left[s_{x_{1}^{-}}, s_{y_{1}^{-}}, s_{z_{1}^{-}}\right],\left[s_{x_{1}^{+}}, s_{y_{1}^{+}}, s_{z_{1}^{+}}\right]\right\rangle ; s_{\varkappa_{1}}, s_{\phi_{1}}\right.$, $\Re_{2}=\left\{\left\langle\left[s_{x_{2}^{-}}, s_{y_{2}^{-}}, s_{z_{2}^{-}}\right],\left[s_{x_{2}^{+}}, s_{y_{2}^{+}}, s_{z_{2}^{+}}\right]\right\rangle ; s_{\varkappa_{2}}, s_{\phi_{2}}, s_{\varphi_{2}}\right\}$ and $\Re_{3}=\left\{\left\langle\left[s_{x_{3}^{-}}, s_{y_{3}^{-}}, s_{z_{3}^{-}}\right],\left[s_{x_{3}^{+}}, s_{y_{3}^{+}}, s_{z_{3}^{+}}\right]\right\rangle ; s_{\varkappa_{3}}, s_{\phi_{3}}, s_{\varphi_{3}}\right\}$ with $\rho \geq 1, \lambda_{1}, \lambda_{2}>0$. Then, we have

(1) $\Re_{1} \oplus \Re_{2}=\Re_{2} \oplus \Re_{1}$

(2) $\Re_{1} \otimes \Re_{2}=\Re_{2} \otimes \Re_{1}$

(3) $\lambda_{1}\left(\Re_{1} \oplus \Re_{2}\right)=\lambda_{1} \Re_{1} \oplus \lambda_{1} \Re_{2}$

(4) $\left(\lambda_{1} \oplus \lambda_{2}\right) \Re_{1}=\lambda_{1} \Re_{1} \oplus \lambda_{2} \Re_{1}$

(5) $\left(\Re_{1} \otimes \Re_{2}\right)^{\lambda_{1}}=\Re_{1}^{\lambda_{1}} \otimes \Re_{2}^{\lambda_{1}}$

(6) $\Re_{1}^{\lambda_{1}} \otimes \Re_{1}^{\lambda_{2}}=\Re_{1}^{\left(\lambda_{1}+\lambda_{2}\right)}$

(7) $\left(\Re_{1} \oplus \Re_{2}\right) \oplus \Re_{3}=\Re_{1} \oplus\left(\Re_{2} \oplus \Re_{3}\right)$

(8) $\left(\Re_{1} \otimes \Re_{2}\right) \otimes \Re_{3}=\Re_{1} \otimes\left(\Re_{2} \otimes \Re_{3}\right)$ 
Proof. (1) Let $\Re_{1}$ and $\Re_{2}$ be the triangular linguistic cubic fuzzy variables and $\lambda_{1}>0$. Then, using the operational laws of addition of two TLCFVs, we have
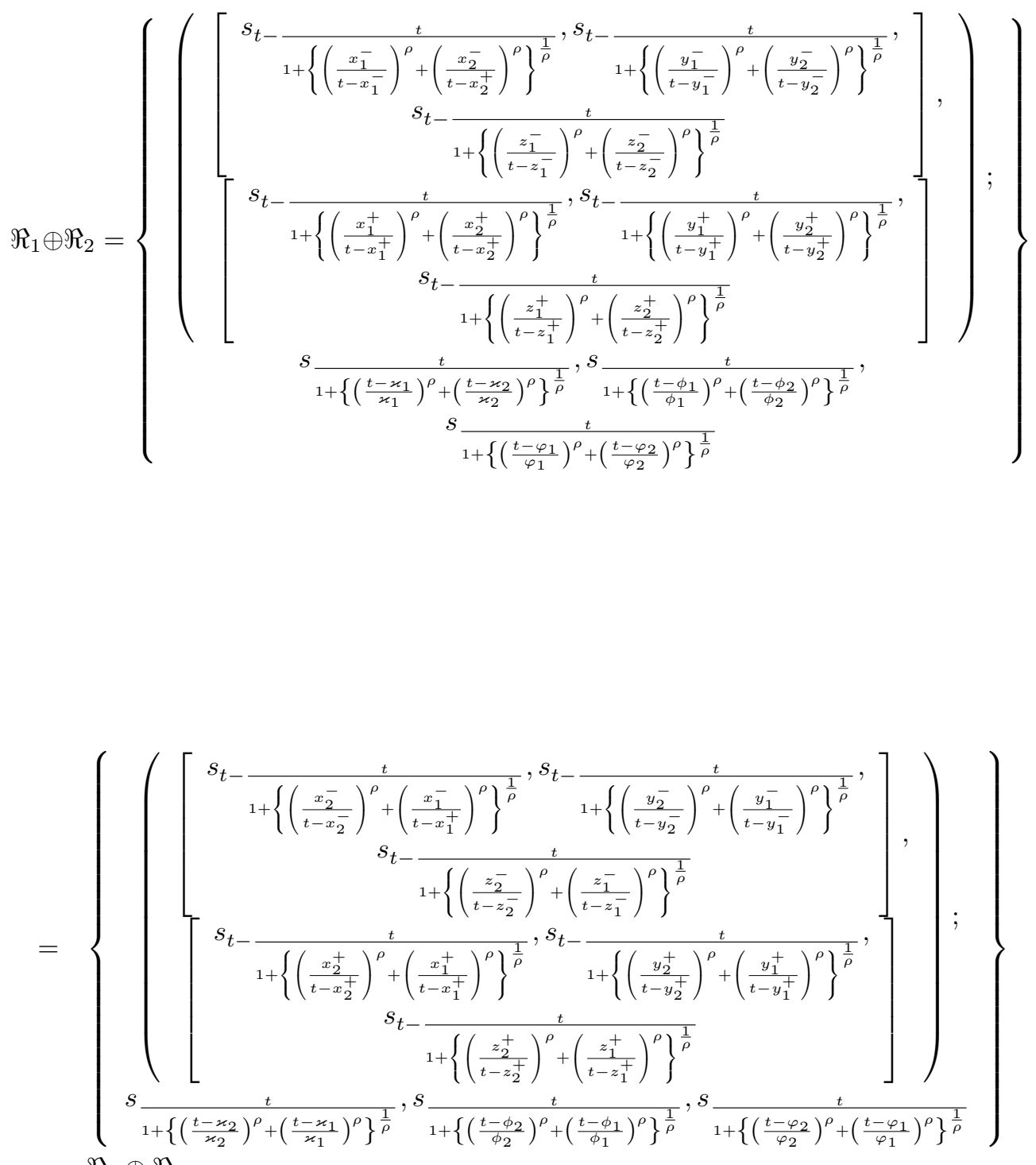

$=\Re_{1} \oplus \Re_{2}$

which show that $\Re_{1} \oplus \Re_{2}=\Re_{2} \oplus \Re_{1}$.

(2) The proof of part (2) is obvious. 
(3) Let $\Re_{1}$ and $\Re_{2}$ be the triangular linguistic cubic fuzzy variables and $\lambda_{1}>0$. Then, we know from the operation laws of TLCFVs,

$$
\begin{aligned}
& \lambda_{1}\left(\Re_{1} \oplus \Re_{2}\right)
\end{aligned}
$$

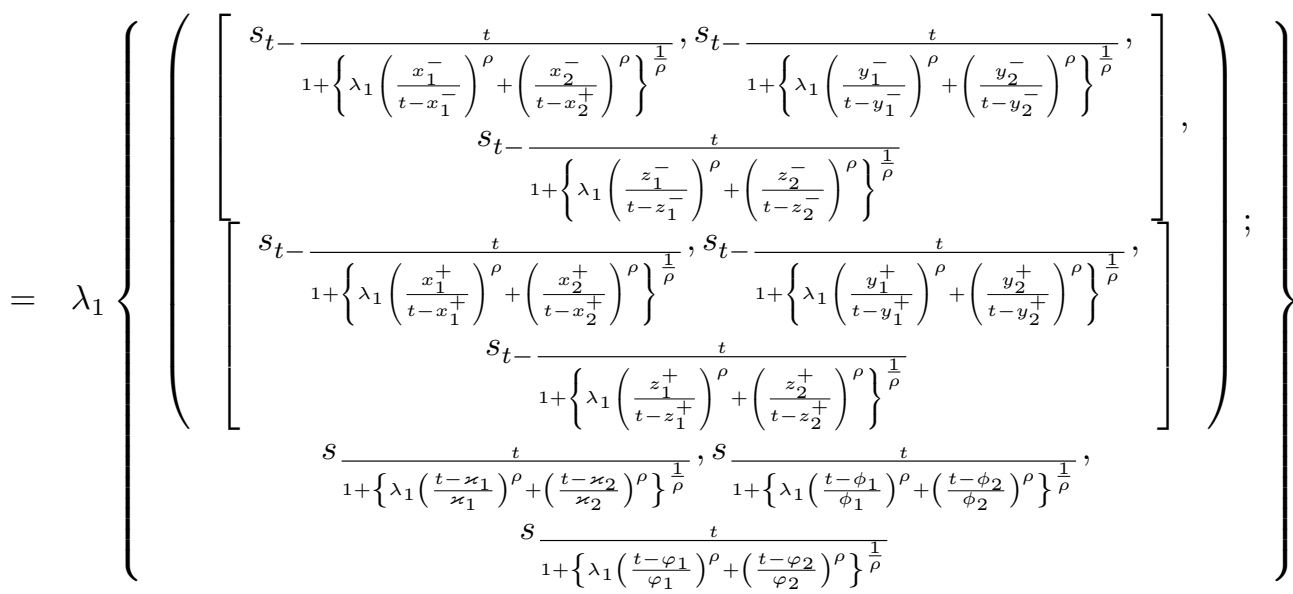

Now, again from the TLCFVs operational laws, we have

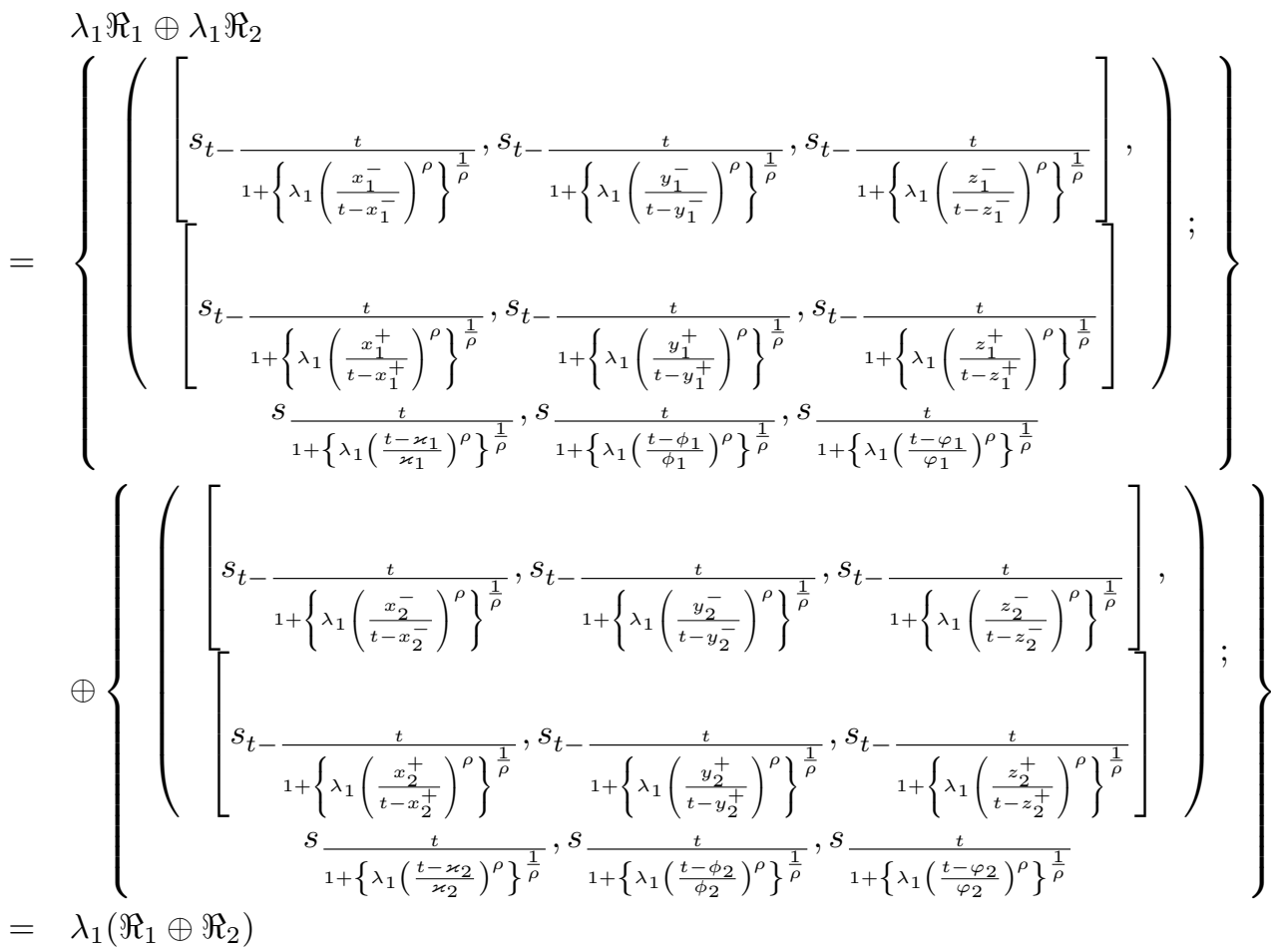

which show that $\lambda_{1}\left(\Re_{1} \oplus \Re_{2}\right)=\lambda_{1} \Re_{1} \oplus \lambda_{1} \Re_{2}$. 
(4) Let $\Re_{1}$ be the triangular linguistic cubic fuzzy variable and $\lambda_{1}, \lambda_{2}>0$. Then, we know from the operation laws of TLCFVs,
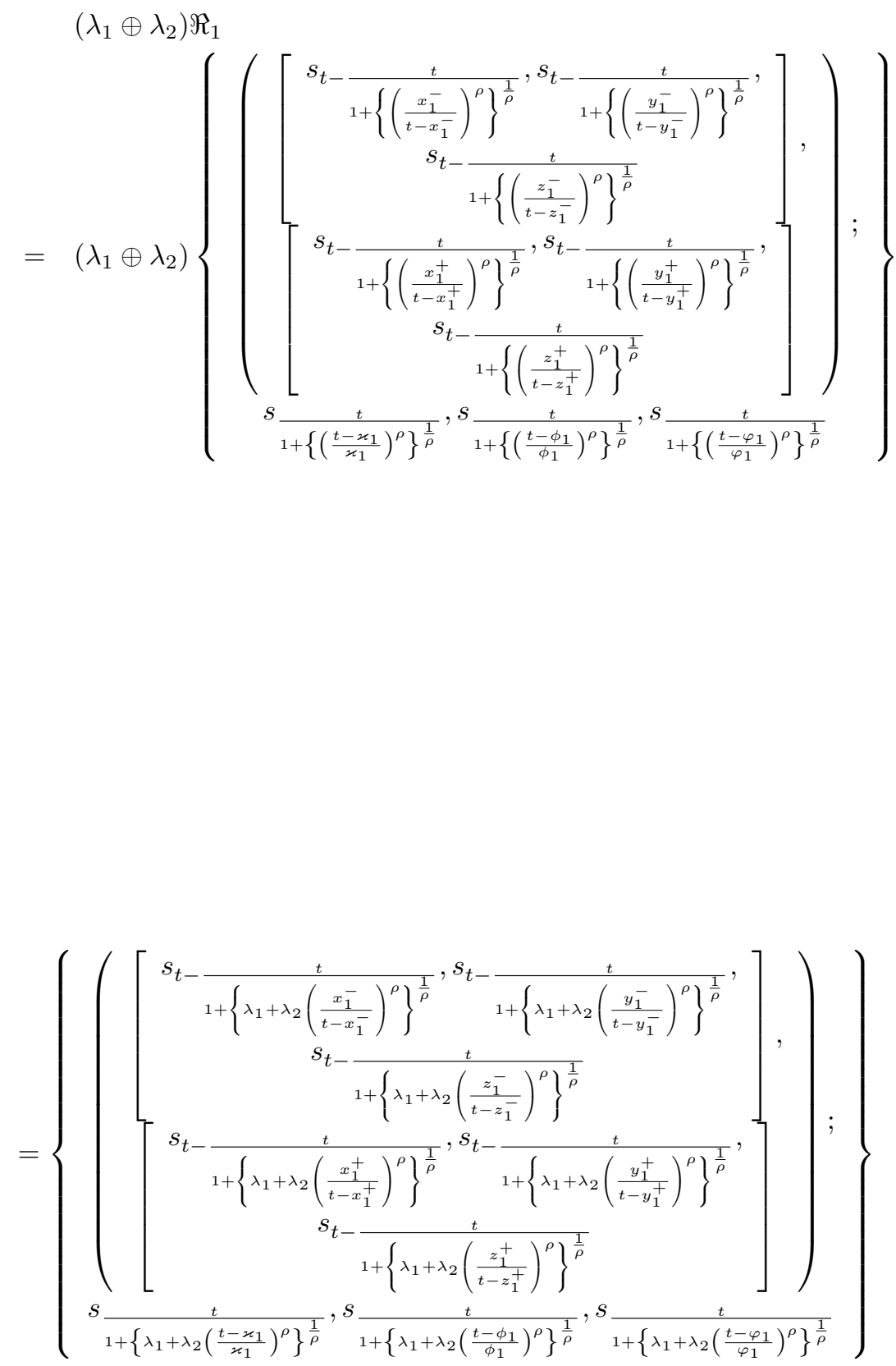
Hence, it proved that $\left(\lambda_{1} \oplus \lambda_{2}\right) \Re_{1}=\lambda_{1} \Re_{1} \oplus \lambda_{2} \Re_{1}$.

(5) Let $\Re_{1}$ and $\Re_{2}$ be the triangular linguistic cubic fuzzy variable and $\lambda_{1}>0$. Then, we know from the operation laws of TLCFVs,

$\left(\Re_{1} \otimes \Re_{2}\right)^{\lambda_{1}}$

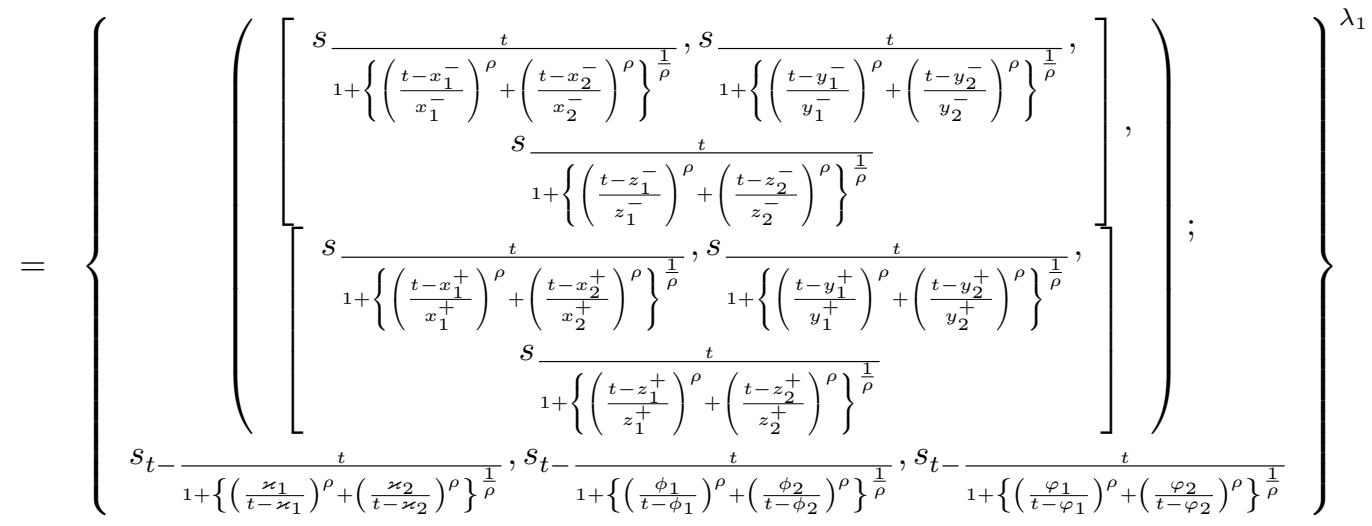


Hence, it proved that $\left(\Re_{1} \otimes \Re_{2}\right)^{\lambda_{1}}=\Re_{1}^{\lambda_{1}} \otimes \Re_{2}^{\lambda_{1}}$. 
(6) Let $\Re_{1}$ and $\Re_{2}$ be the triangular linguistic cubic fuzzy variable and $\lambda_{1}, \lambda_{2}>$ 0 . Then, we know from the operation laws of TLCFVs,

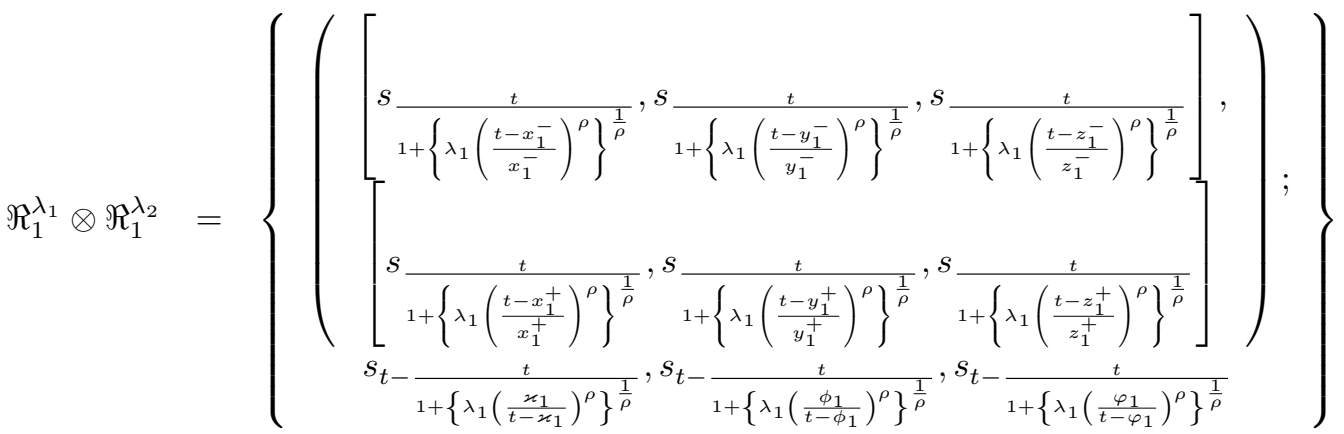

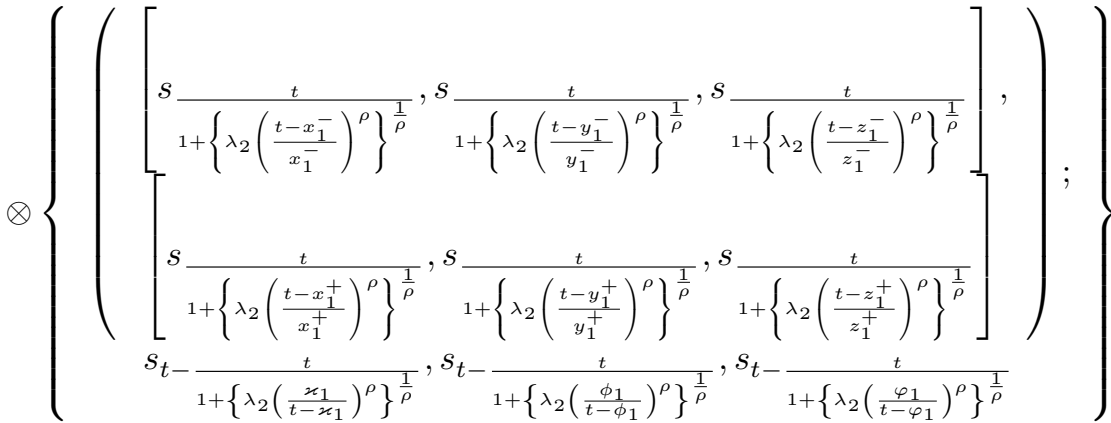

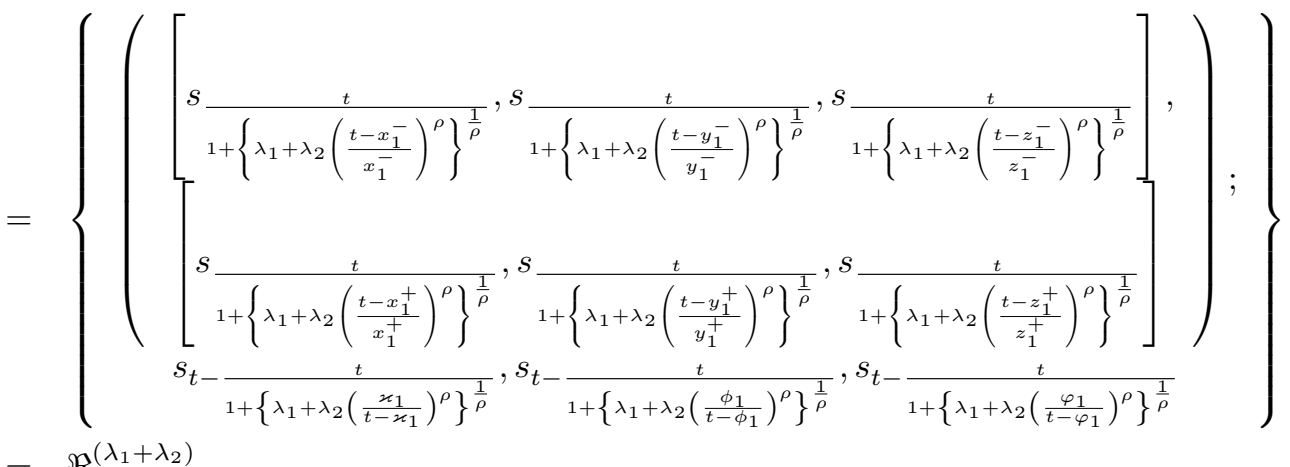$$
=\Re_{1}^{\left(\lambda_{1}+\lambda_{2}\right)}
$$

Hence, it proved that $\Re_{1}^{\lambda_{1}} \otimes \Re_{1}^{\lambda_{2}}=\Re_{1}^{\left(\lambda_{1}+\lambda_{2}\right)}$.

(7) Let $\Re_{1}, \Re_{2}$ and $\Re_{2}$ be the triangular linguistic cubic fuzzy variables and $\lambda_{1}>0$. Then, using the operational laws of addition of two TLCFVs, we 
have

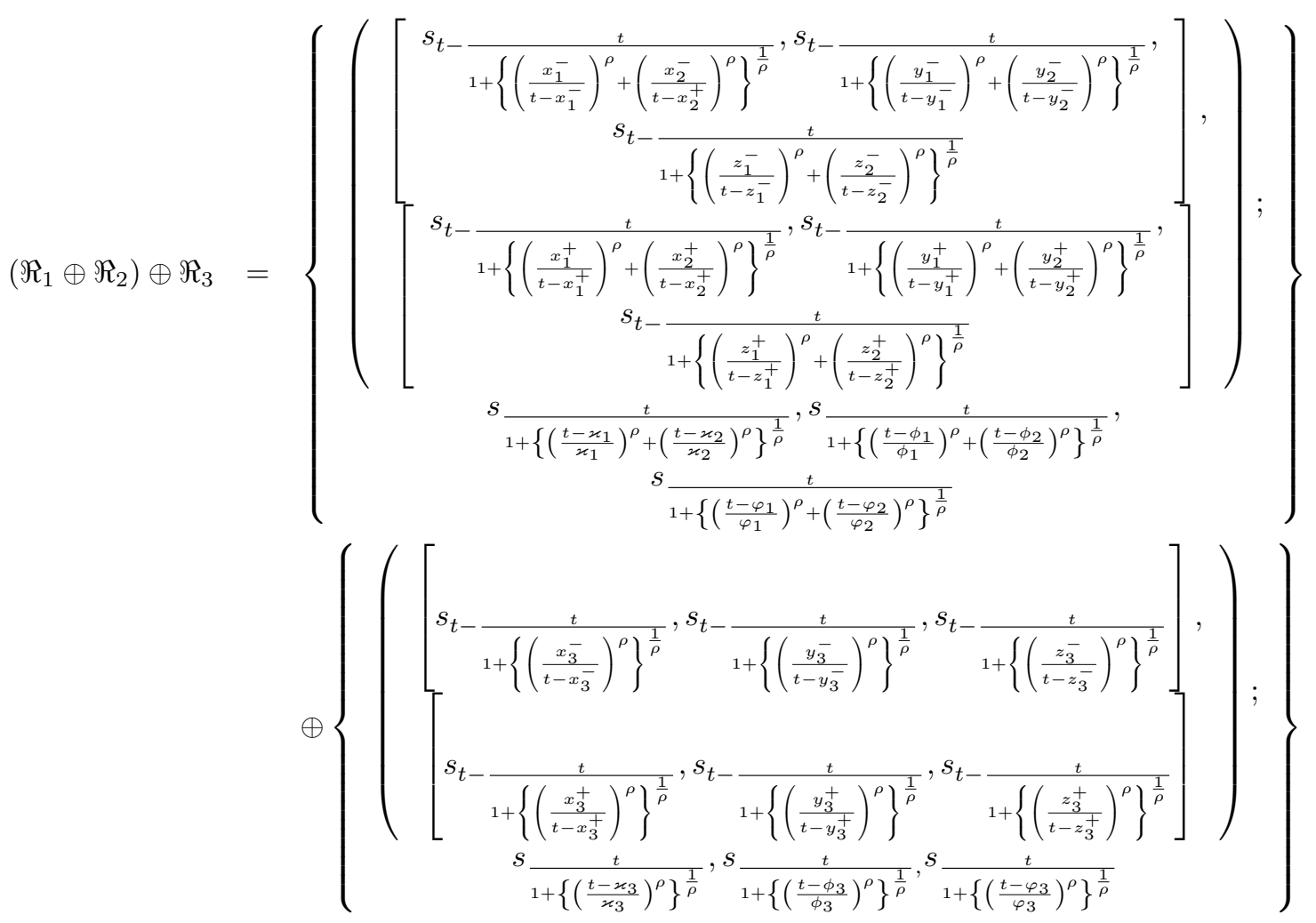

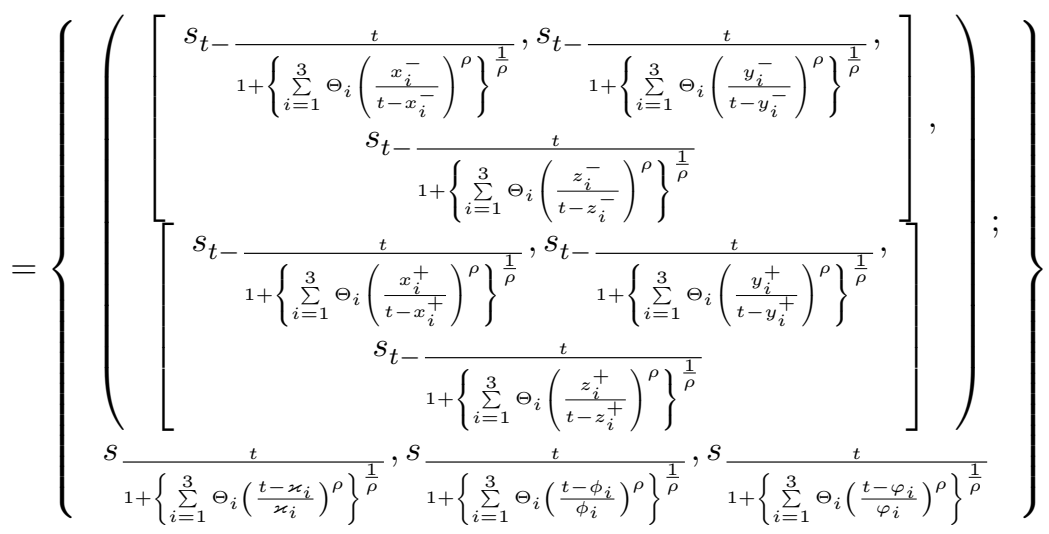


Now,

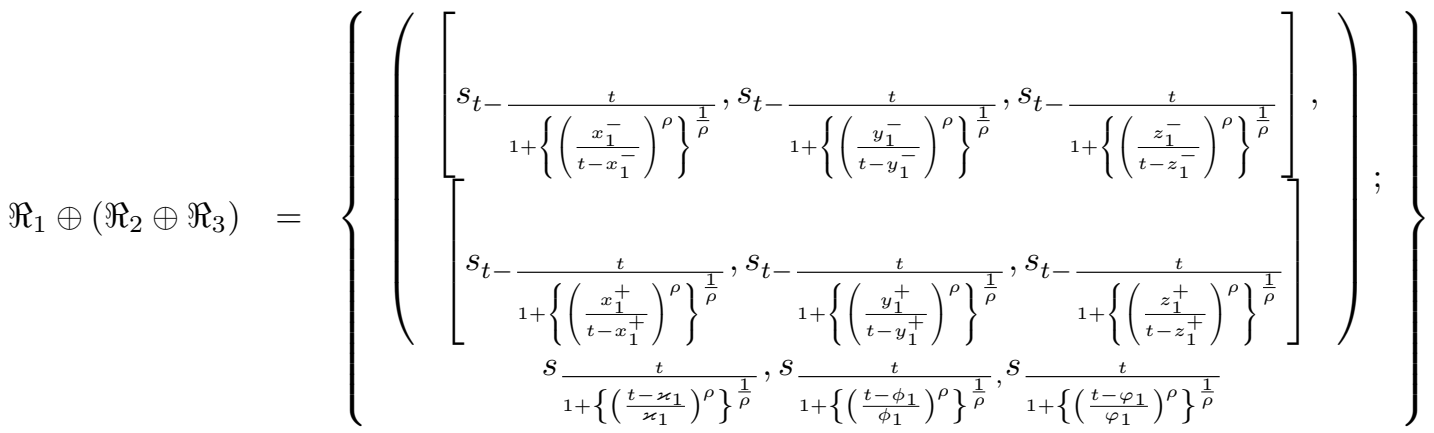

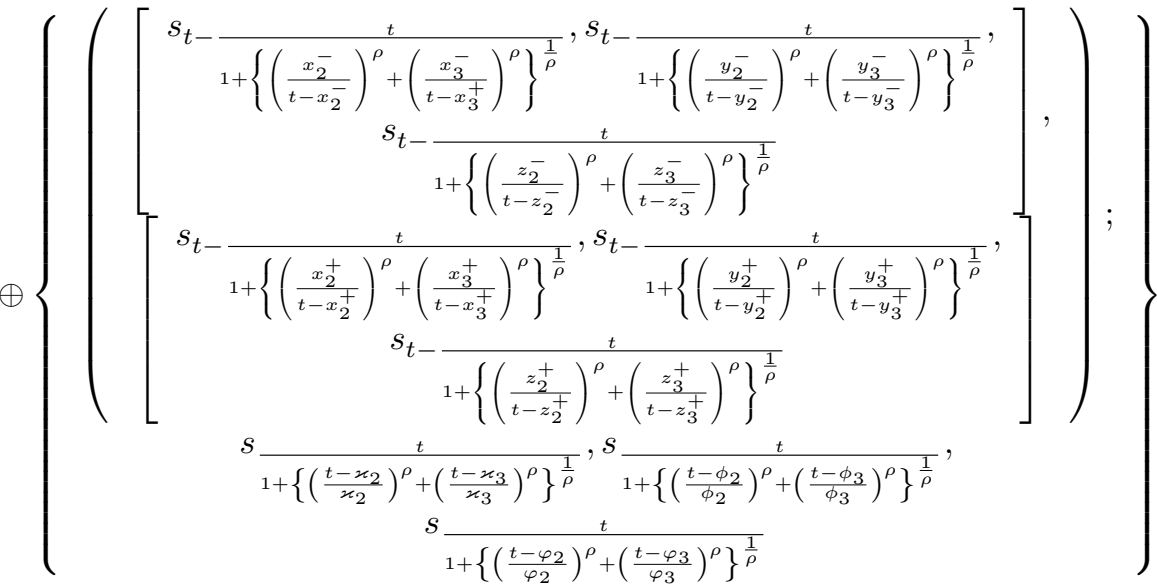

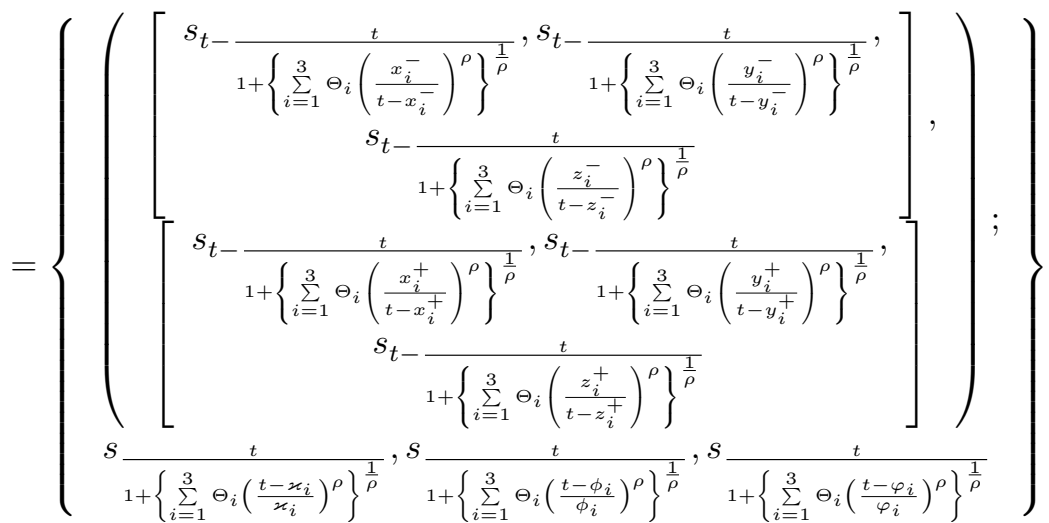

Which show that $\left(\Re_{1} \oplus \Re_{2}\right) \oplus \Re_{3}=\Re_{1} \oplus\left(\Re_{2} \oplus \Re_{3}\right)$.

(8) The proof part (8) is same as part (7).

4. Triangular Linguistic Cubic Fuzzy Dombi Aggregation Operators

\subsection{Triangular Linguistic Cubic Fuzzy Dombi Averaging Aggregation} Operators.

Definition 9. Let $\Re_{i}=\left\{\left\langle\left[s_{x_{i}^{-}}, s_{y_{i}^{-}}, s_{z_{i}^{-}}\right],\left[s_{x_{i}^{+}}, s_{y_{i}^{+}}, s_{z_{i}^{+}}\right]\right\rangle ; s_{\varkappa_{i}}, s_{\phi_{i}}, s_{\varphi_{i}}\right\}(i=1, \ldots, n)$ be a collection of triangular linguistic cubic fuzzy variables. Then, the triangular 
linguistic cubic fuzzy Dombi weighted average (TLCFDWA) operator is described as;

$$
\operatorname{TLCFDW} A_{\Theta}\left(\Re_{1}, \ldots, \Re_{n}\right)=\sum_{i=1}^{n}\left(\Theta_{i} \Re_{i}\right) .
$$

Where the weights of $\Re_{i}(i=1, \ldots, n)$ are $\Theta=\left(\Theta_{1}, \ldots, \Theta_{n}\right)^{T}$, with $\Theta_{i}>0$ and $\sum_{i=1}^{n} \Theta_{i}=1$.

Theorem 2. Let $\Re_{i}=\left\{\left\langle\left[s_{x_{i}^{-}}, s_{y_{i}^{-}}, s_{z_{i}^{-}}\right],\left[s_{x_{i}^{+}}, s_{y_{i}^{+}}, s_{z_{i}^{+}}\right]\right\rangle ; s_{\varkappa_{i}}, s_{\phi_{i}}, s_{\varphi_{i}}\right\}(i=1, \ldots, n)$ be a collection of triangular linguistic cubic fuzzy variables. Then, their aggregated value is also a TLCFVs using the TLCFDWA operator, and

$$
\begin{aligned}
& T L C F D W A_{\Theta}\left(\Re_{1}, \ldots, \Re_{n}\right)=\sum_{i=1}^{n}\left(\Theta_{i} \Re_{i}\right)
\end{aligned}
$$

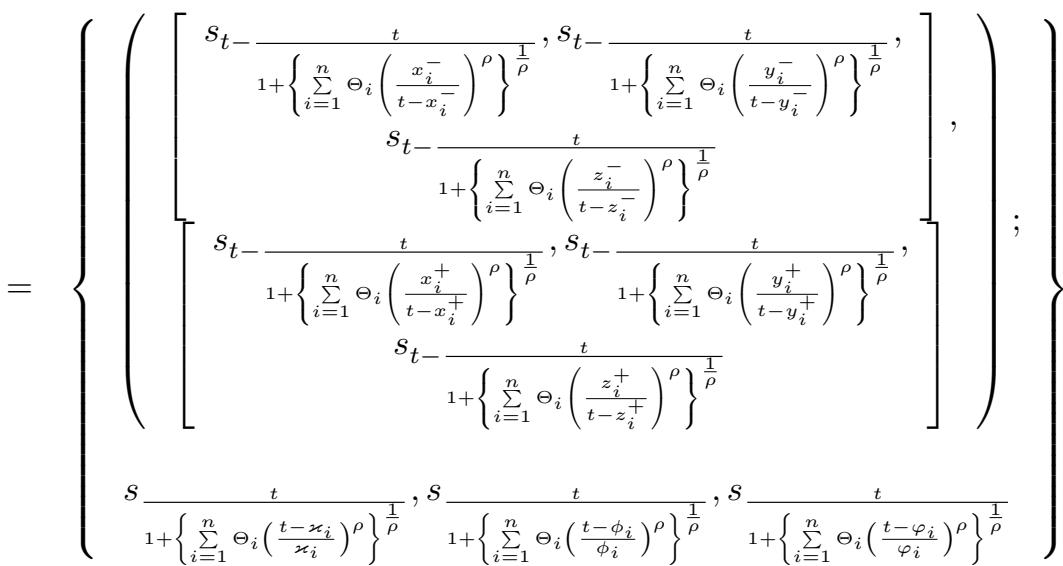

Where $\Theta=\left(\Theta_{1}, \ldots, \Theta_{n}\right)^{T}$ be the weight vector of $\Re_{i}(i=1, \ldots, n)$, with $\Theta_{i}>0$ and $\sum_{i=1}^{n} \Theta_{i}=1$.

Proof. By using the mathematical induction, we have;

(i) If $n=2$, then by using Dombi operation of TLCFVs, we have

$$
\operatorname{TLCFDWA} A_{\Theta}\left(\Re_{1}, \Re_{2}\right)=\left(\Re_{1} \oplus \Re_{2}\right)
$$

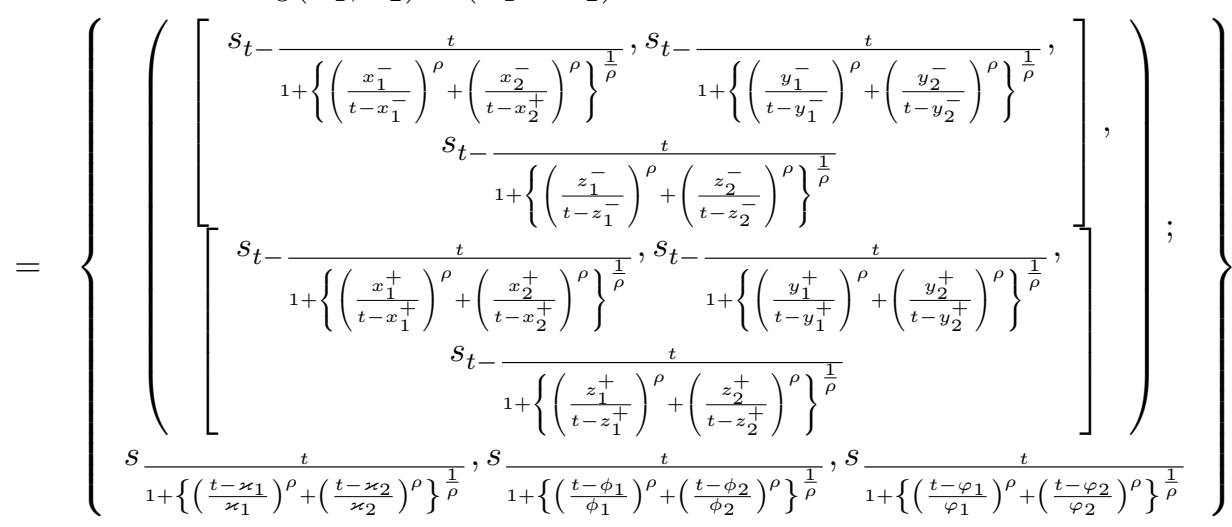


MUHAMMAD QIYAS*, SALEEM ABDULLAH, RONNASON CHINRAM, AND MUNEEZA

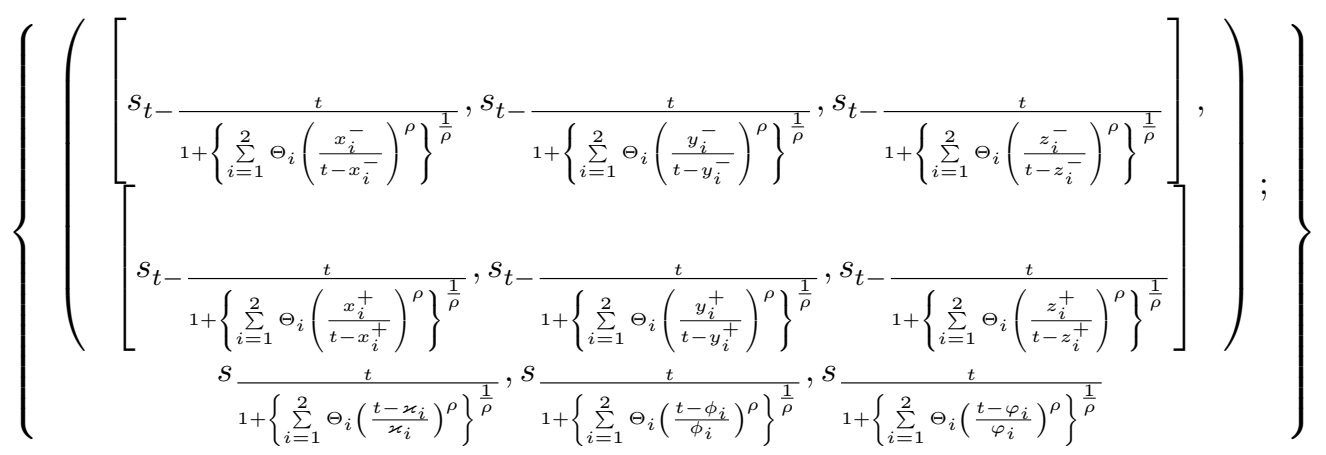

Hence, Equ. (4.2) is true for $n=2$.

Let Equ. (4.2) is hold for $n=k$. Then, by the Equ. (4.2), we get

$$
\begin{aligned}
& T L C V D W A_{\Theta}\left(\Re_{1}, \ldots, \Re_{k}\right)=\sum_{i=1}^{k}\left(\Theta_{i} \Re_{i}\right)
\end{aligned}
$$

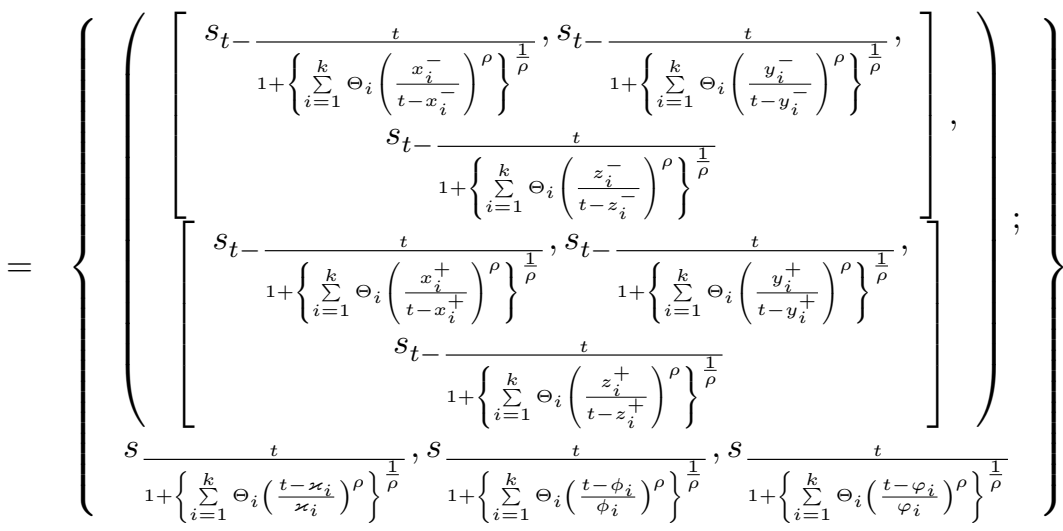


Now, for $n=k+1$, we get

$$
\begin{aligned}
& T L C F D W A_{\Theta}\left(\Re_{1}, \ldots, \Re_{k}, \Re_{k+1}\right)=\sum_{i=1}^{n} \Theta_{i} \Re_{i} \oplus \Theta_{k+1} \Re_{k+1}
\end{aligned}
$$

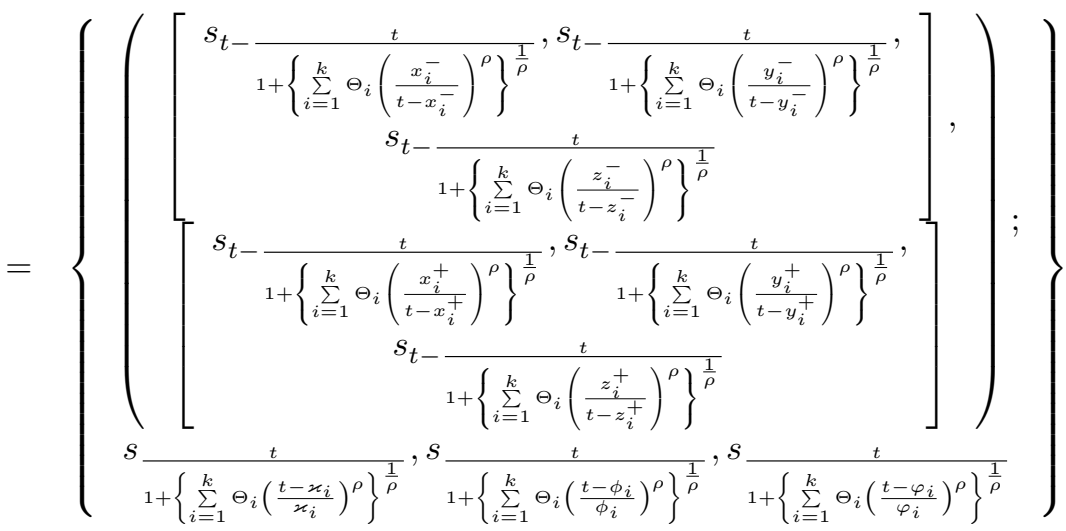

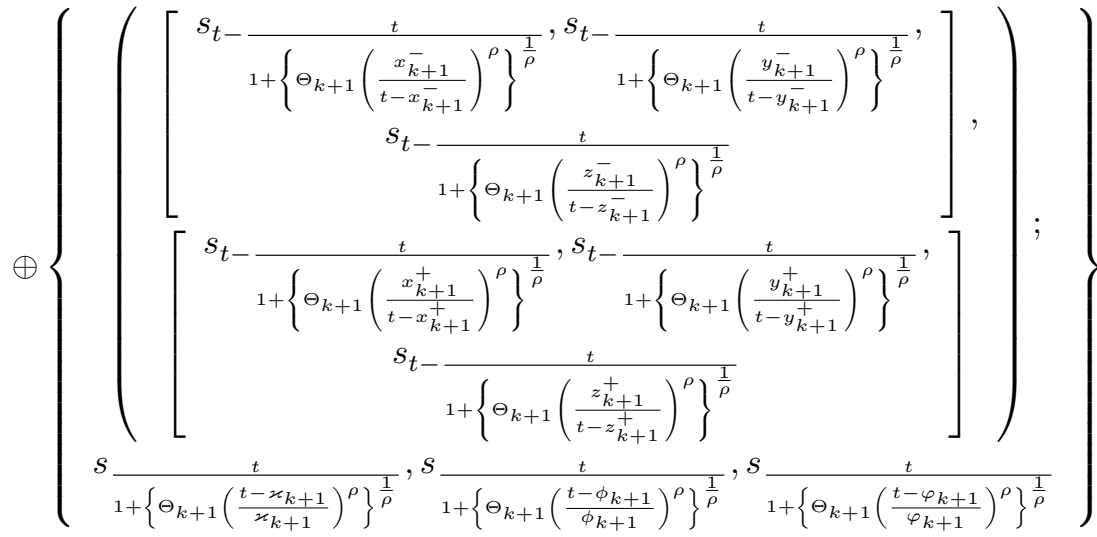

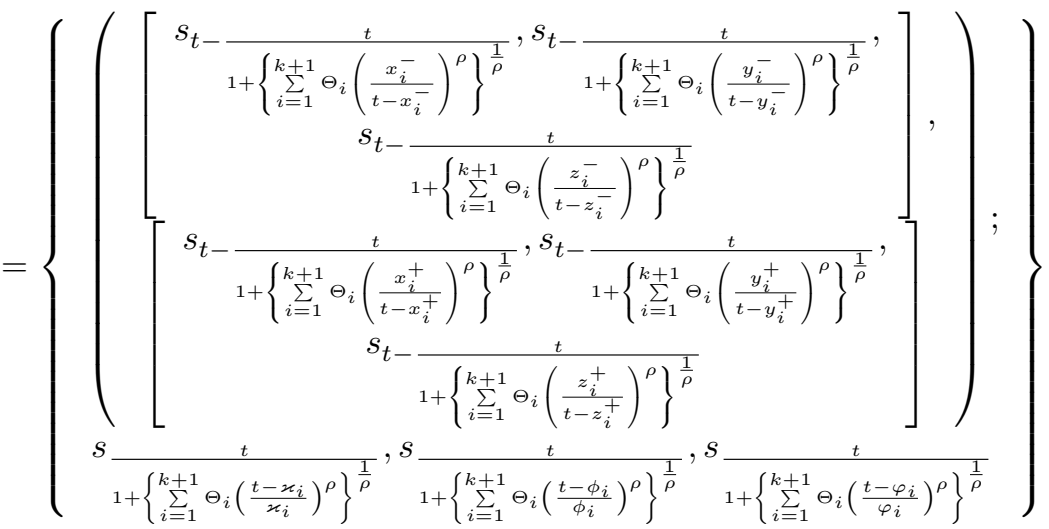

Thus, Equ. (4.2) is true for all $n=k+1$. Which is required.

Theorem 3. (Idempotency) Let $\Re_{i}=\left\{\left\langle\left[s_{x_{i}^{-}}, s_{y_{i}^{-}}, s_{z_{i}^{-}}\right],\left[s_{x_{i}^{+}}, s_{y_{i}^{+}}, s_{z_{i}^{+}}\right]\right\rangle ; s_{\varkappa_{i}}, s_{\phi_{i}}, s_{\varphi_{i}}\right\}(i=$ $1, \ldots, n)$ be a family of triangular linguistic cubic fuzzy variables, with the weight vector are $\Theta=\left(\Theta_{1}, \ldots, \Theta_{n}\right)^{T}$ with $\Theta_{i}>0$ and $\sum_{i=1}^{n} \Theta_{i}=1$, all are identical i.e., $\Re_{i}=\Re$. 
Then,

$$
T L C F D W A_{\Theta}\left(\Re_{1}, \ldots, \Re_{n}\right)=\Re .
$$

Proof. Sience, $\Re_{i}=\left\{\left\langle\left[s_{x_{i}^{-}}, s_{y_{i}^{-}}, s_{z_{i}^{-}}\right],\left[s_{x_{i}^{+}}, s_{y_{i}^{+}}, s_{z_{i}^{+}}\right]\right\rangle ; s_{\varkappa_{i}}, s_{\phi_{i}}, s_{\varphi_{i}}\right\}(i=1, \ldots, n)$. Then, by Equ. (4.4), we have

$$
\begin{aligned}
& \operatorname{TLCFDW} A_{\Theta}\left(\Re_{1}, \ldots, \Re_{n}\right)=\sum_{i=1}^{n}\left(\Theta_{i} \Re_{i}\right)
\end{aligned}
$$

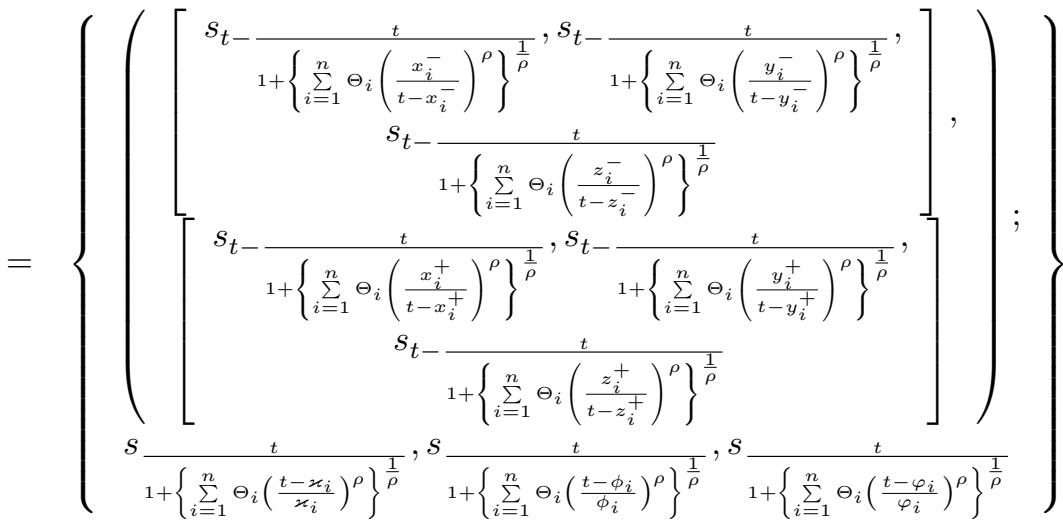

Since, for all $i, \Re_{i}=\Re$, i.e., $x_{i}^{-}=x^{-}, y_{i}^{+}=y^{+}, y_{i}^{-}=y^{-}, x_{i}^{+}=x^{+}, z_{i}^{-}=$ $z^{-}, z_{i}^{+}=z^{+}$

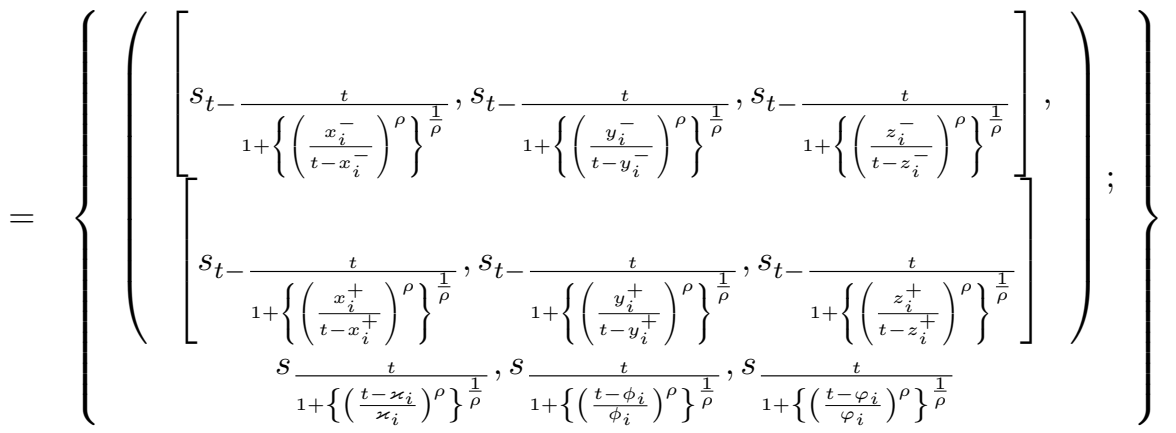

$$
\begin{aligned}
& =\left\{\left\langle\left[s_{x^{-}}, s_{y^{-}}, s_{z^{-}}\right],\left[s_{x^{+}}, s_{y^{+}}, s_{z^{+}}\right]\right\rangle, s_{\varkappa}, s_{\phi}, s_{\varphi}\right\} \\
& =\Re
\end{aligned}
$$

Theorem 4. (Boundedness) Let $\Re_{i}=\left\{\left\langle\left[s_{x_{i}^{-}}, s_{y_{i}^{-}}, s_{z_{i}^{-}}\right],\left[s_{x_{i}^{+}}, s_{y_{i}^{+}}, s_{z_{i}^{+}}\right]\right\rangle ; s_{\varkappa_{i}}, s_{\phi_{i}}, s_{\varphi_{i}}\right\}(i=$ $1, \ldots, n)$ are the family of triangular linguistic cubic fuzzy variables, and the weight vector of $\Re_{i}$ are $\Theta=\left(\Theta_{1}, \ldots, \Theta_{n}\right)^{T}$, with $\Theta_{i}>0$ and $\sum_{i=1}^{n} \Theta_{i}=1$. Then,

$$
\Re^{*} \leq T L C F D W A_{\Theta}\left(\Re_{1}, \ldots, \Re_{n}\right) \leq \Re^{* *} .
$$


Proof. Let $\Re^{*}=\min \left(\Re_{1}, \ldots, \Re_{n}\right)$ and $\Re^{* *}=\max \left(\Re_{1}, \ldots, \Re_{n}\right)$. Then, we have

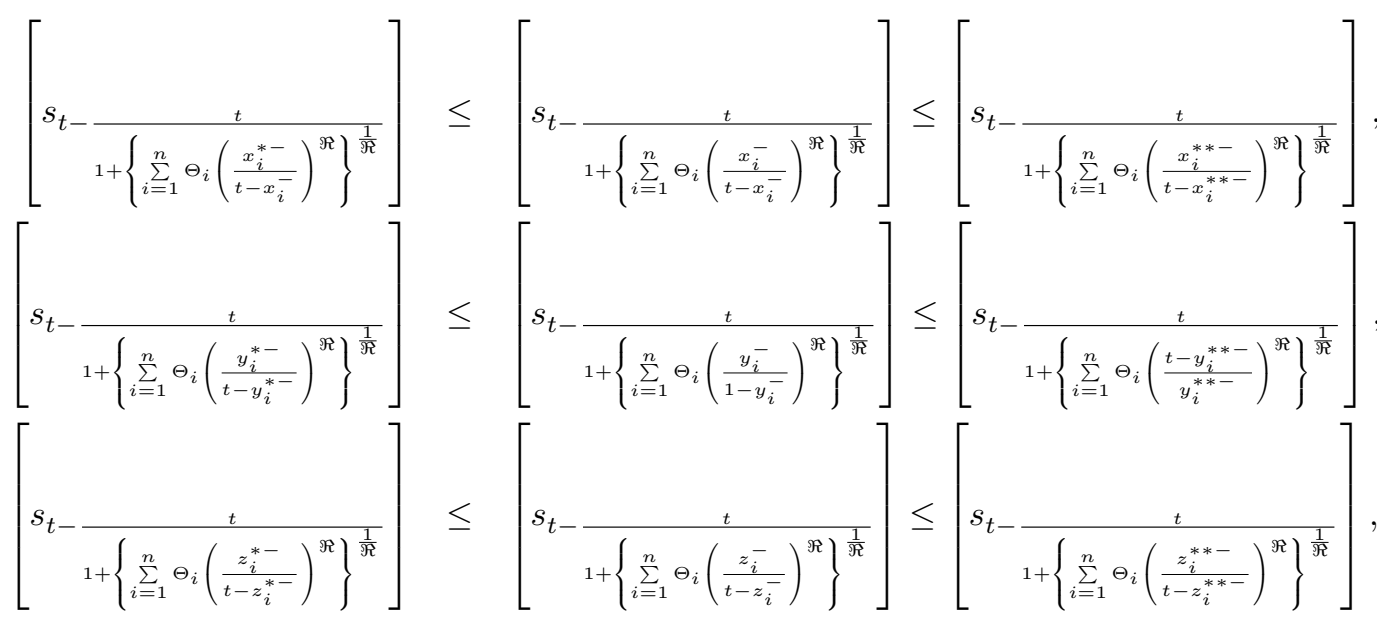

and

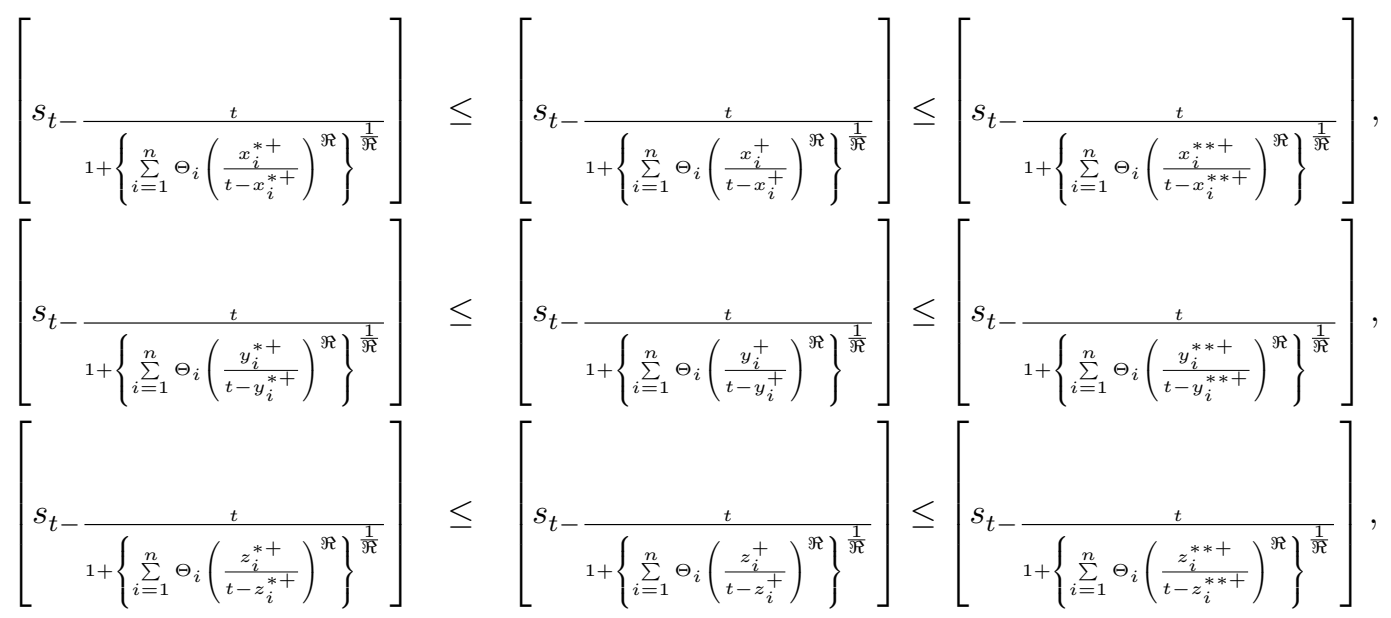

Therefore,

$$
\Re^{*} \leq \operatorname{LICFDWA} A_{\Theta}\left(\Re_{1}, \ldots, \Re_{n}\right) \leq \Re^{* *}
$$

Theorem 5. (Monotonicity) Let $\Re_{i}=\left\{\left\langle\left[s_{x_{i}^{-}}, s_{y_{i}^{-}}, s_{z_{i}^{-}}\right],\left[s_{x_{i}^{+}}, s_{y_{i}^{+}}, s_{z_{i}^{+}}\right]\right\rangle ; s_{\varkappa_{i}}, s_{\phi_{i}}, s_{\varphi_{i}}\right\}(i=$ $1, \ldots, n)$ are the family of triangular linguistic cubic fuzzy variables, and the weight vector of $\Re_{i}$ are $\Theta=\left(\Theta_{1}, \ldots, \Theta_{n}\right)^{T}$, with $\Theta_{i}>0$ and $\sum_{i=1}^{n} \Theta_{i}=1$. Then,

$$
T L C F D W A_{\Theta}\left(\Re_{1}, \ldots, \Re_{n}\right) \leq T L C F D W A_{\Theta}\left(\Re_{1}^{*}, \ldots, \Re_{n}^{*}\right) .
$$

Proof. Science, $\Re_{i} \leq \Re_{i}^{*}, \forall i=1, \ldots, n$, there exist $\sum_{i=1}^{n} \Theta_{i} \Re_{i} \leq \sum_{i=1}^{n} \Theta_{i} \Re_{i}^{*}$. Hence,

$$
T L C F D W A_{\Theta}\left(\Re_{1}, \ldots, \Re_{n}\right) \leq T L C F D W A_{\Theta}\left(\Re_{1}^{*}, \ldots, \Re_{n}^{*}\right) .
$$

Where $\Re_{i}^{*}(i=1, \ldots, n)$ are the permutation of $\Re_{i}(i=1, \ldots, n)$. 
Definition 10. Let $\Re_{i}=\left\{\left\langle\left[s_{x_{i}^{-}}, s_{y_{i}^{-}}, s_{z_{i}^{-}}\right],\left[s_{x_{i}^{+}}, s_{y_{i}^{+}}, s_{z_{i}^{+}}\right]\right\rangle ; s_{\varkappa_{i}}, s_{\phi_{i}}, s_{\varphi_{i}}\right\}(i=1, \ldots, n)$ are the family of triangular linguistic cubic fuzzy variables. A triangular linguistic cubic fuzzy Dombi order weighted average (TLCFDOWA) operator is a function TLCFDOWA $: \Omega^{n} \rightarrow \Omega$, and is defined as below,

$$
T L C F D O W A_{\Theta}\left(\Re_{1}, \ldots, \Re_{n}\right)=\Theta_{1} \Re_{\sigma_{(1)}} \oplus \ldots \oplus \Theta_{i} \Re_{\sigma_{(i)}} .
$$

Then, the operator TLCFDOWA is called triangular linguistic cubic fuzzy Dombi order weighted average operator of dimension $n, \forall i, \Re_{\sigma_{(i-1)}} \geq \Re_{\sigma_{(i)}}\left(\sigma_{(1)}, \ldots, \sigma_{(n)}\right)$ is the permutation of $(1, \ldots, n)$. Also, the weighting vector of $\Re_{i}(i=1, \ldots, n)$ is $\Theta=\left(\Theta_{1}, \ldots, \Theta_{n}\right)^{T}$ with $\sum_{i=1}^{n} \Theta_{i}=1$ and $\Theta_{i} \in[0,1]$. Moreover, the TLCFDOWA operator converts to the TLCFDA operator of dimension $n$ if $\varpi=(1 / n, \ldots, 1 / n)^{T}$, which is given as,

$$
\operatorname{TLCFDOW} A_{\Theta}\left(\Re_{1}, \ldots, \Re_{n}\right)=1 / n\left(\Re_{1} \oplus \ldots \oplus \Re_{n}\right) .
$$

Theorem 6. Let $\Re_{i}=\left\{\left\langle\left[s_{x_{i}^{-}}, s_{y_{i}^{-}}, s_{z_{i}^{-}}\right],\left[s_{x_{i}^{+}}, s_{y_{i}^{+}}, s_{z_{i}^{+}}\right]\right\rangle ; s_{\varkappa_{i}}, s_{\phi_{i}}, s_{\varphi_{i}}\right\}(i=1, \ldots, n)$ are the family of triangular linguistic cubic fuzzy variables. Then, their aggregated value by utilizing the TLCFDOWA operator is also a TLCFV and as;

$$
\begin{aligned}
& T L C F D O W A_{\Theta}\left(\Re_{1}, \ldots, \Re_{n}\right)=\sum_{i=1}^{n}\left(\Theta_{i} \Re_{\sigma(i)}\right)
\end{aligned}
$$

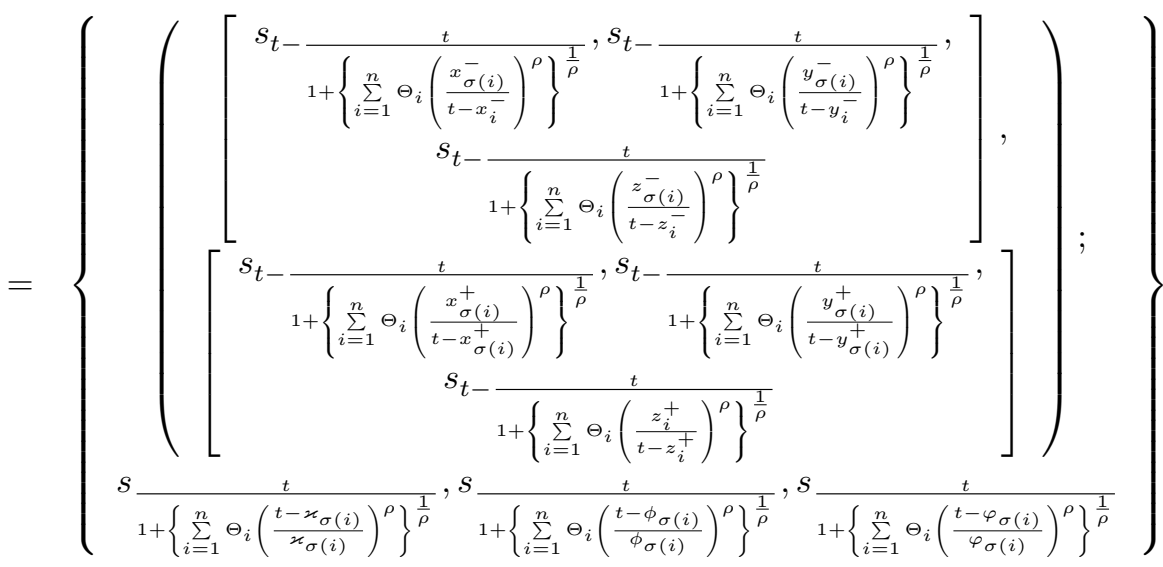

Also, the weight vector of $\Re_{i}(i=1, \ldots, n)$ is $\Theta=\left(\Theta_{1}, \ldots, \Theta_{n}\right)^{T}$ with $\sum_{i=1}^{n} \Theta_{i}=1$ and $\Theta_{i} \in[0,1]$, the order represented by permutation $\left(\sigma_{(1)}, \ldots, \sigma_{(n)}\right)$ of $(i=1, \ldots, n)$ subject to $\Re_{\sigma_{(i-1)}} \geq \Re_{\sigma_{(i)}}$ for all $(i=1, \ldots, n)$.

Definition 11. Let $\Re_{i}=\left\{\left\langle\left[s_{x_{i}^{-}}, s_{y_{i}^{-}}, s_{z_{i}^{-}}\right],\left[s_{x_{i}^{+}}, s_{y_{i}^{+}}, s_{z_{i}^{+}}\right]\right\rangle ; s_{\varkappa_{i}}, s_{\phi_{i}}, s_{\varphi_{i}}\right\}(i=1, \ldots, n)$ are the family of triangular linguistic cubic fuzzy variables. A triangular linguistic cubic fuzzy hybrid average (TLCFDHA) operator is a function TLCFDHA : $\Omega^{n} \rightarrow \Omega$, with the associated weight vector $\varpi=\left(\varpi_{1}, \ldots, \varpi_{n}\right)^{T}$ and $\sum_{i=1}^{n} \varpi_{i}=1$ and $\varpi_{i} \in[0,1]$, such that,

$$
T L C F D H A_{\Theta, \varpi}\left(\Re_{1}, \ldots, \Re_{n}\right)=\Theta_{1} \widetilde{\Re}_{\sigma_{(1)}} \oplus \ldots \oplus \Theta_{i} \widetilde{\Re}_{\sigma_{(i)}} .
$$


Here, $\widetilde{\Re}_{\sigma_{(i)}}$ is the $i^{\text {th }}$ biggest value of the weighted triangular linguistic cubic fuzzy values $\Re_{i}$. Also, the weight of $\Re_{i}$ is $\Theta=\left(\Theta_{1}, \ldots, \Theta_{n}\right)^{T}$ with $\sum_{i=1}^{n} \Theta_{i}=1$ and $\Theta_{i} \in$ $[0,1]$, i.e., $\widetilde{\Re}_{i}=n \varpi_{i} \Re_{i}=\left\{\left\langle\left[s_{\widetilde{x}_{i}^{-}}, s_{\widetilde{y}_{i}^{-}}, s_{\widetilde{z}_{i}^{-}}\right],\left[s_{\widetilde{x}_{i}^{+}}, s_{\widetilde{y}_{i}^{+}}, s_{\widetilde{z}_{i}^{+}}\right]\right\rangle ; s_{\widetilde{\varkappa}_{i}}, s_{\widetilde{\phi}_{i}}, s_{\widetilde{\varphi}_{i}}\right\}(i=$

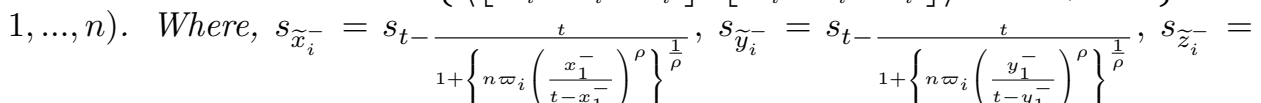

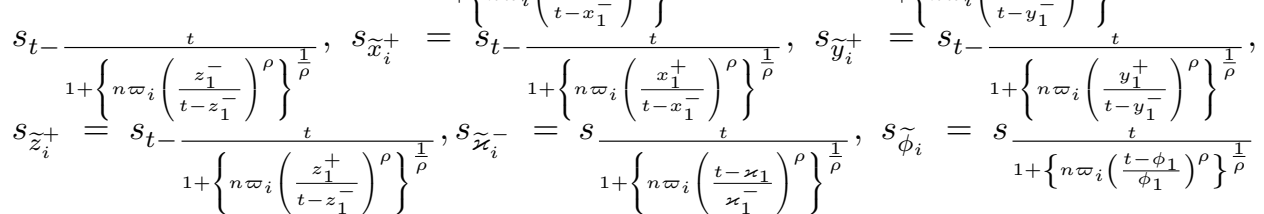
and $s_{\widetilde{\varphi}_{i}}=s \frac{t}{1+\left\{n \varpi_{i}\left(\frac{t-\varphi_{1}}{\varphi_{1}}\right)^{\rho}\right\}^{\frac{1}{\rho}}}$. Here, $n$ is the balancing coefficient, especially the TLCFDHA operator will get the form of a triangular linguistic cubic fuzzy Dombi average (TLCFDHA) operator of dimension $n$ if $\varpi=(1 / n, \ldots, 1 / n)^{T}$.

Theorem 7. Let $\Re_{i}=\left\{\left\langle\left[s_{x_{i}^{-}}, s_{y_{i}^{-}}, s_{z_{i}^{-}}\right],\left[s_{x_{i}^{+}}, s_{y_{i}^{+}}, s_{z_{i}^{+}}\right]\right\rangle ; s_{\varkappa_{i}}, s_{\phi_{i}}, s_{\varphi_{i}}\right\}(i=1, \ldots, n)$ are the family of triangular linguistic cubic fuzzy variables. Then, their aggregated value by utilizing the TLCFDHA operator is also a TLCFV and as;

$$
\begin{aligned}
& T L C F D H A_{\Theta, \varpi}\left(\Re_{1}, \ldots, \Re_{n}\right)=\sum_{i=1}^{n}\left(\Theta_{i} \widetilde{\Re}_{\sigma(i)}\right)
\end{aligned}
$$

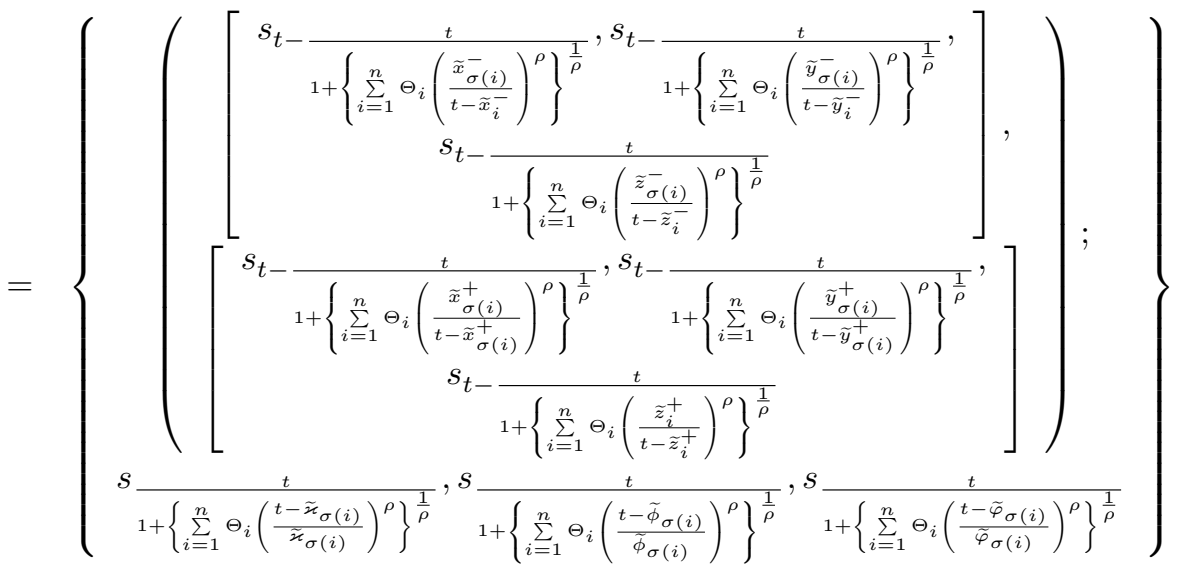

Also, the weight vector of $\Re_{i}(i=1, \ldots, n)$ is $\Theta=\left(\Theta_{1}, \ldots, \Theta_{n}\right)^{T}$ with $\sum_{i=1}^{n} \Theta_{i}=1$ and $\Theta_{i} \in[0,1]$, the order represented by permutation $\left(\sigma_{(1)}, \ldots, \sigma_{(n)}\right)$ of $(i=1, \ldots, n)$ subject to $\Re_{\sigma_{(i-1)}} \geq \Re_{\sigma_{(i)}}$ for all $(i=1, \ldots, n)$.

\subsection{Triangular Linguistic Cubic Fuzzy Dombi Geometric Aggregation Operators.}

Definition 12. Let $\Re_{i}=\left\{\left\langle\left[s_{x_{i}^{-}}, s_{y_{i}^{-}}, s_{z_{i}^{-}}\right],\left[s_{x_{i}^{+}}, s_{y_{i}^{+}}, s_{z_{i}^{+}}\right]\right\rangle ; s_{\varkappa_{i}}, s_{\phi_{i}}, s_{\varphi_{i}}\right\}(i=1, \ldots, n)$ be a collection of triangular linguistic cubic fuzzy variables. Then, the triangular linguistic cubic fuzzy Dombi weighted geometric (TLCFDWA) operator is described 
as;

$$
T L C F D W G_{\Theta}\left(\Re_{1}, \ldots, \Re_{n}\right)=\prod_{i=1}^{n}\left(\Re_{i}\right)^{\Theta_{i}} .
$$

Where the weights of $\Re_{i}(i=1, \ldots, n)$ are $\Theta=\left(\Theta_{1}, \ldots, \Theta_{n}\right)^{T}$, with $\Theta_{i}>0$ and $\sum_{i=1}^{n} \Theta_{i}=1$.

Theorem 8. Let $\Re_{i}=\left\{\left\langle\left[s_{x_{i}^{-}}, s_{y_{i}^{-}}, s_{z_{i}^{-}}\right],\left[s_{x_{i}^{+}}, s_{y_{i}^{+}}, s_{z_{i}^{+}}\right]\right\rangle ; s_{\varkappa_{i}}, s_{\phi_{i}}, s_{\varphi_{i}}\right\}(i=1, \ldots, n)$ be a collection of triangular linguistic cubic fuzzy variables. Then, their aggregated value is also a TLCFVs using the TLCFDWA operator, and

$$
T L C F D W G_{\Theta}\left(\Re_{1}, \ldots, \Re_{n}\right)=\prod_{i=1}^{n}\left(\Re_{i}\right)^{\Theta_{i}}
$$

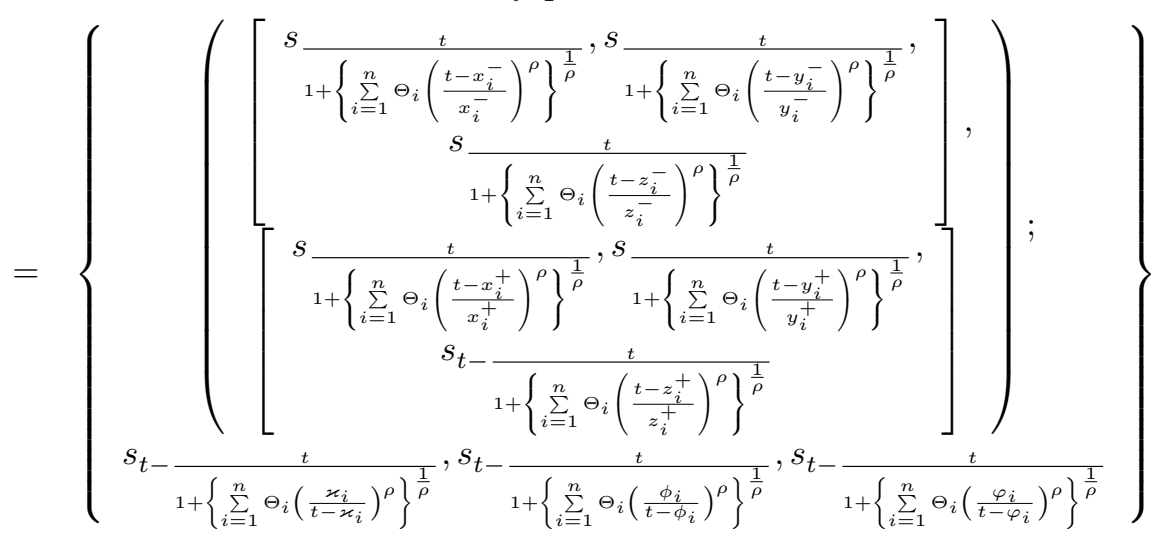

Where $\Theta=\left(\Theta_{1}, \ldots, \Theta_{n}\right)^{T}$ be the weights of $\Re_{i}(i=1, \ldots, n)$, with $\Theta_{i}>0$ and $\sum_{i=1}^{n} \Theta_{i}=1$.

Proof. By using the mathematical induction, we have;

(i) If $n=2$, then by using Dombi operation on TLCFVs, we have

$$
\begin{aligned}
& T L C F D W G_{\Theta}\left(\Re_{1}, \Re_{2}\right)=\left(\Re_{1} \otimes \Re_{2}\right)
\end{aligned}
$$

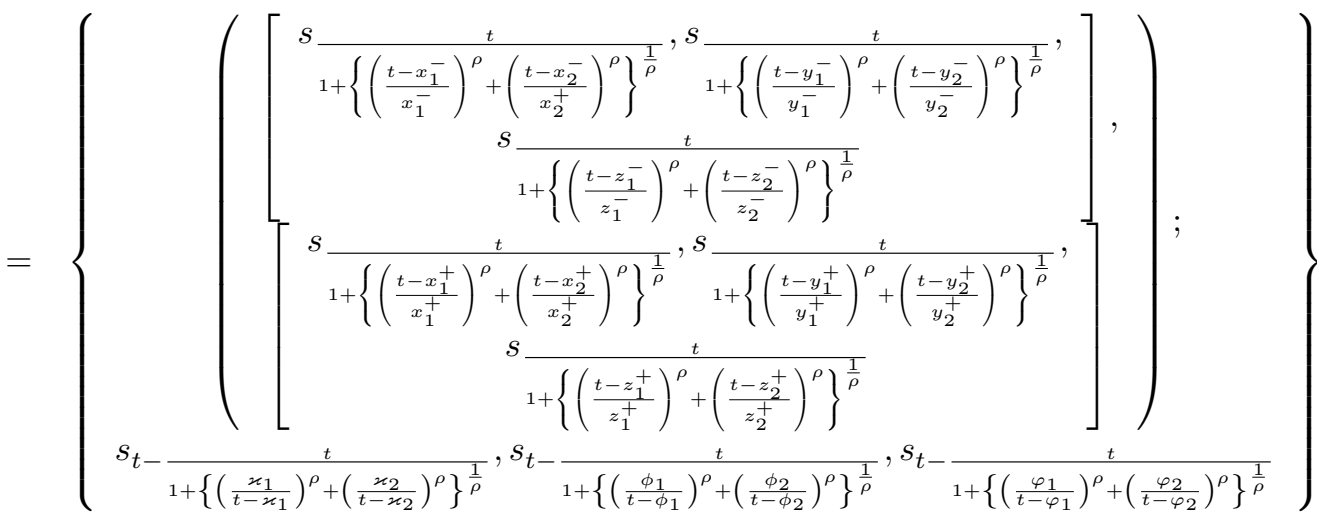




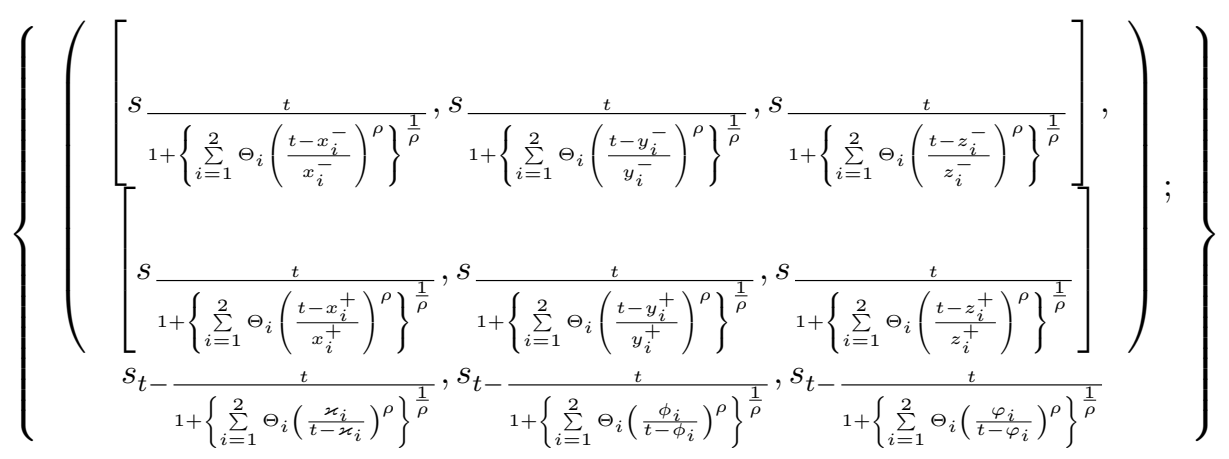

Hence, Equ. (4.12) is true for $n=2$.

Let Equ. (4.12) is hold for $n=k$. Then, by the Equ. (4.12), we get

$$
\begin{aligned}
& T L C F D W G_{\Theta}\left(\Re_{1}, \ldots, \Re_{k}\right)=\sum_{i=1}^{n}(\Re)^{\Theta_{i}}
\end{aligned}
$$

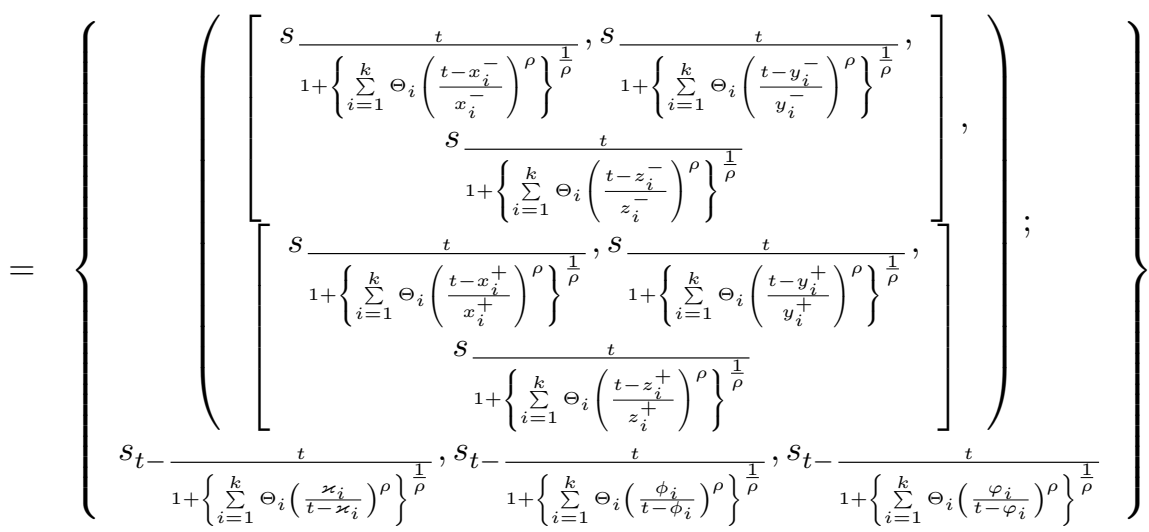

Now, for $n=k+1$, we get

$$
T L C F D W G_{\Theta}\left(\Re_{1}, \ldots, \Re_{k}, \Re_{k+1}\right)=\prod_{i=1}^{n}\left(\Re_{i}\right)^{\Theta_{i}} \otimes\left(\Re_{k+1}\right)^{\Theta_{k+1}}
$$




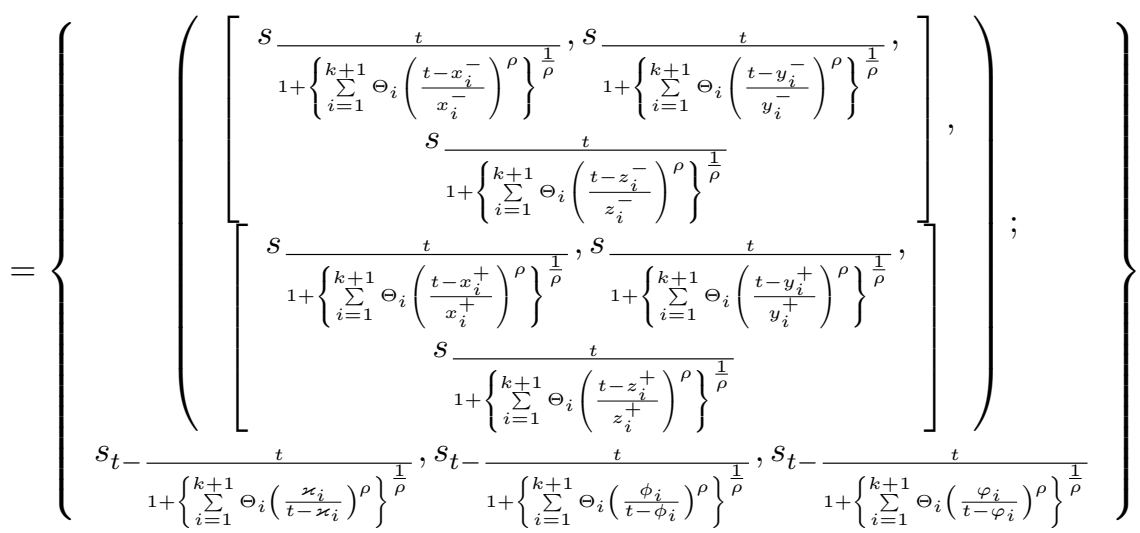

Thus, Equ. (4.12) is true for all $n=k+1$. Which is needed.

Theorem 9. (Idempotency) Let $\Re_{i}=\left\{\left\langle\left[s_{x_{i}^{-}}, s_{y_{i}^{-}}, s_{z_{i}^{-}}\right],\left[s_{x_{i}^{+}}, s_{y_{i}^{+}}, s_{z_{i}^{+}}\right]\right\rangle ; s_{\varkappa_{i}}, s_{\phi_{i}}, s_{\varphi_{i}}\right\}(i=$ $1, \ldots, n)$ be a family of triangular linguistic cubic fuzzy variables, with the weight vector are $\Theta=\left(\Theta_{1}, \ldots, \Theta_{n}\right)^{T}$ with $\Theta_{i}>0$ and $\sum_{i=1}^{n} \Theta_{i}=1$, all are identical i.e., $\Re_{i}=\Re$. Then,

$$
T L C V D W G_{\Theta}\left(\Re_{1}, \ldots, \Re_{n}\right)=\Re .
$$


Proof. Since, $\Re_{i}=\left\{\left\langle\left[s_{x_{i}^{-}}, s_{y_{i}^{-}}, s_{z_{i}^{-}}\right],\left[s_{x_{i}^{+}}, s_{y_{i}^{+}}, s_{z_{i}^{+}}\right]\right\rangle ; s_{\varkappa_{i}}, s_{\phi_{i}}, s_{\varphi_{i}}\right\}(i=1, \ldots, n)$. Then, by Equ. (4.4), we have

$$
\begin{aligned}
& T L C F D W G_{\Theta}\left(\Re_{1}, \ldots, \Re_{n}\right)=\prod_{i=1}^{n}\left(\Re_{i}\right)^{\Theta_{i}}
\end{aligned}
$$

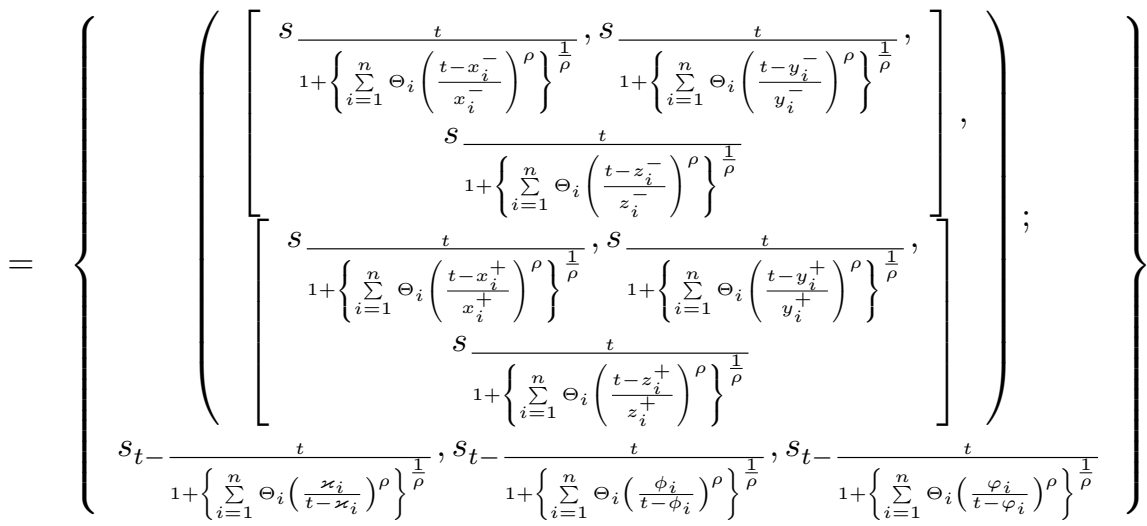

Since, for all $i, \Re_{i}=\Re$, i.e., $x_{i}^{-}=x^{-}, y_{i}^{+}=y^{+}, y_{i}^{-}=y^{-}, x_{i}^{+}=x^{+}, z_{i}^{-}=$ $z^{-}, z_{i}^{+}=z^{+}$

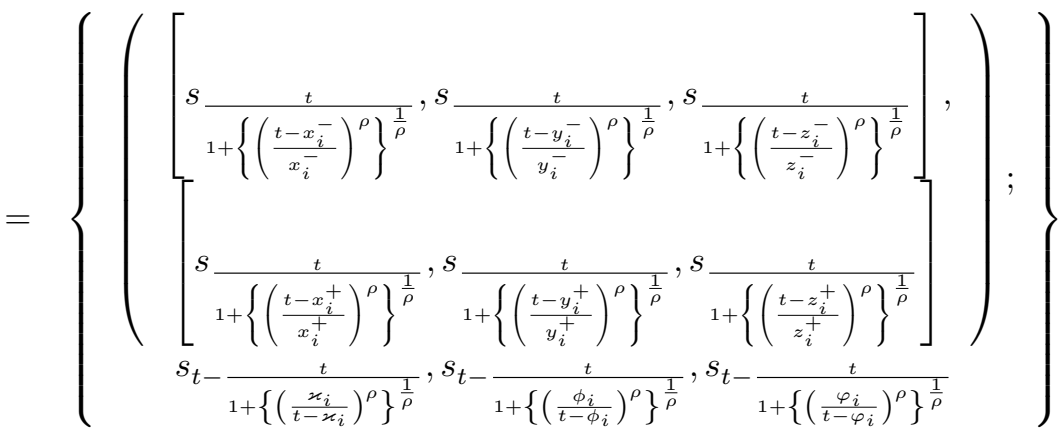

$$
\begin{aligned}
& =\left\{\left\langle\left[s_{x^{-}}, s_{y^{-}}, s_{z^{-}}\right],\left[s_{x^{+}}, s_{y^{+}}, s_{z^{+}}\right]\right\rangle ; s_{\varkappa}, s_{\phi}, s_{\varphi}\right\} \\
& =\Re
\end{aligned}
$$

Theorem 10. (Boundedness) Let $\Re_{i}=\left\{\left\langle\left[s_{x_{i}^{-}}, s_{y_{i}^{-}}, s_{z_{i}^{-}}\right],\left[s_{x_{i}^{+}}, s_{y_{i}^{+}}, s_{z_{i}^{+}}\right]\right\rangle ; s_{\varkappa_{i}}, s_{\phi_{i}}, s_{\varphi_{i}}\right\}(i=$ $1, \ldots, n)$ are the family of triangular linguistic cubic fuzzy variables, and the weight vector of $\Re_{i}$ are $\Theta=\left(\Theta_{1}, \ldots, \Theta_{n}\right)^{T}$, with $\Theta_{i}>0$ and $\sum_{i=1}^{n} \Theta_{i}=1$. Then,

$$
\Re^{*} \leq T L C F D W G_{\Theta}\left(\Re_{1}, \ldots, \Re_{n}\right) \leq \Re^{* *} .
$$


Proof. Let $\Re^{*}=\min \left(\Re_{1}, \ldots, \Re_{n}\right)$ and $\Re^{* *}=\max \left(\Re_{1}, \ldots, \Re_{n}\right)$. Then, we have

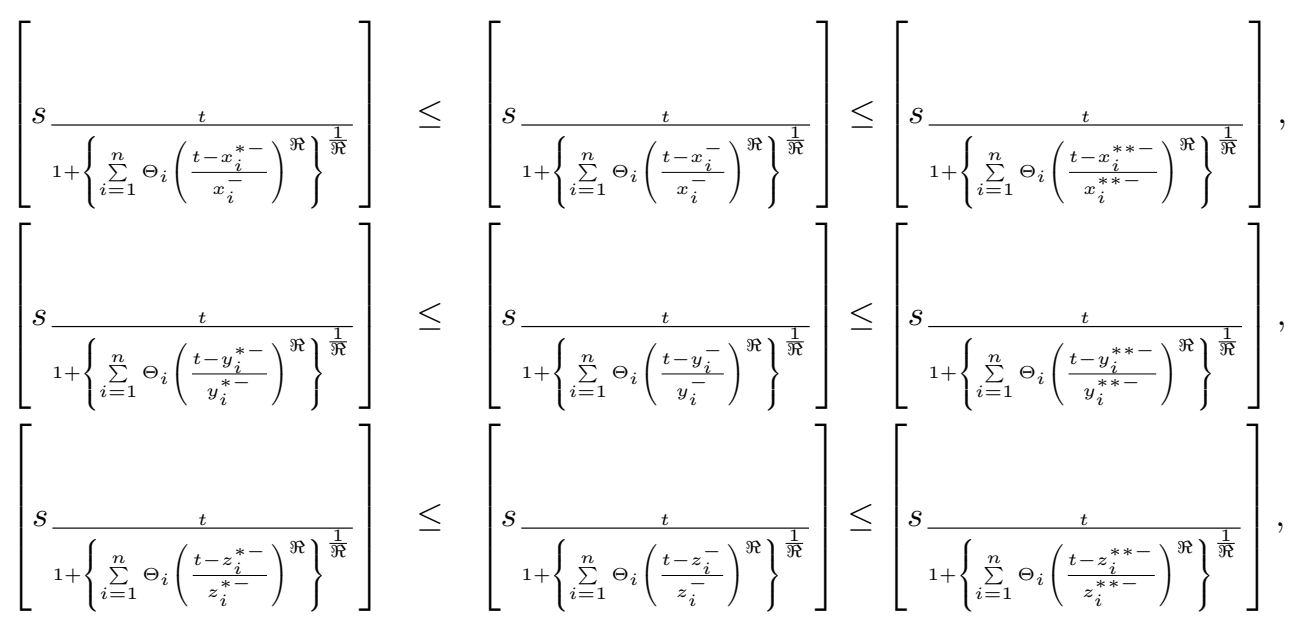

and

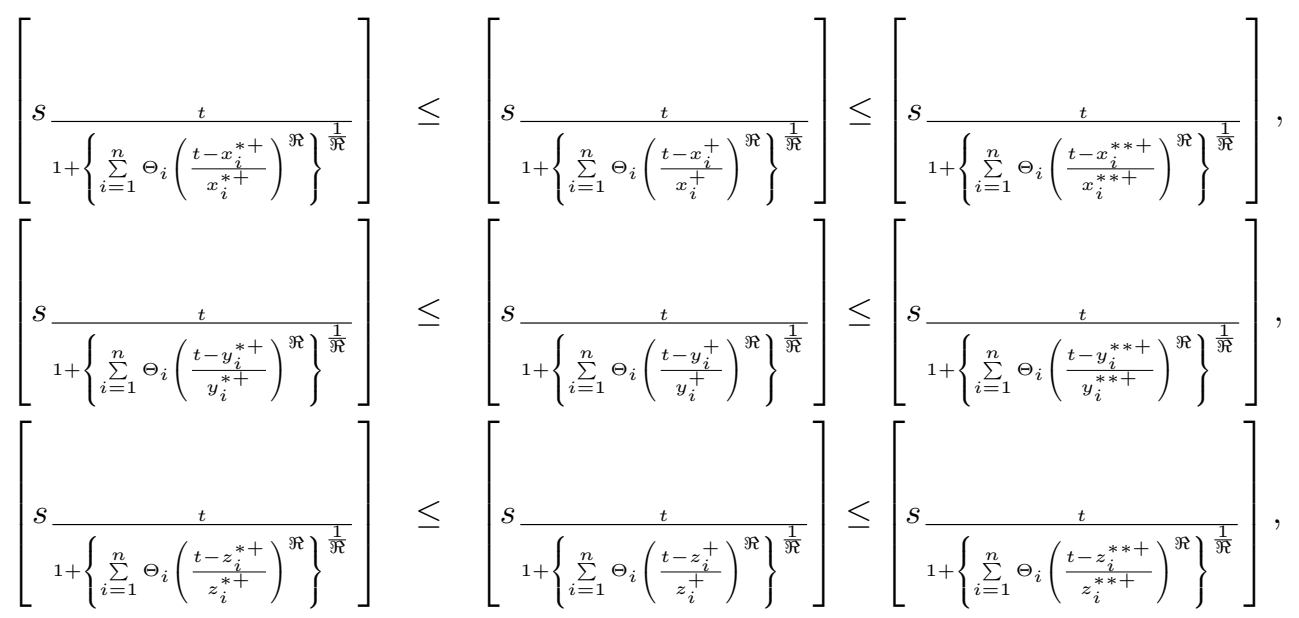

Therefore,

$$
\Re^{*} \leq T L C F D W G_{\Theta}\left(\Re_{1}, \ldots, \Re_{n}\right) \leq \Re^{* *}
$$

Theorem 11. (Monotonicity) Let $\Re_{i}=\left\{\left\langle\left[s_{x_{i}^{-}}, s_{y_{i}^{-}}, s_{z_{i}^{-}}\right],\left[s_{x_{i}^{+}}, s_{y_{i}^{+}}, s_{z_{i}^{+}}\right]\right\rangle ; s_{\varkappa_{i}}, s_{\phi_{i}}, s_{\varphi_{i}}\right\}(i=$ $1, \ldots, n)$ are the family of triangular linguistic cubic fuzzy variables and the weight vector of $\Re_{i}$ are $\Theta=\left(\Theta_{1}, \ldots, \Theta_{n}\right)^{T}$ with $\Theta_{i}>0$ and $\sum_{i=1}^{n} \Theta_{i}=1$. Then,

$$
T L C F D W G_{\Theta}\left(\Re_{1}, \ldots, \Re_{n}\right) \leq T L C F D W G_{\Theta}\left(\Re_{1}^{*}, \ldots, \Re_{n}^{*}\right) .
$$

Proof. Science, $\Re_{i} \leq \Re_{i}^{*}, \forall i=1, \ldots, n$, there exist $\sum_{i=1}^{n} \Theta_{i} \Re_{i} \leq \sum_{i=1}^{n} \Theta_{i} \Re_{i}^{*}$. Hence,

$$
T L C F D W G_{\Theta}\left(\Re_{1}, \ldots, \Re_{n}\right) \leq T L C F D W G_{\Theta}\left(\Re_{1}^{*}, \ldots, \Re_{n}^{*}\right) .
$$

Where, $\Re_{i}^{*}(i=1, \ldots, n)$ are the permutation of $\Re_{i}(i=1, \ldots, n)$. 
Definition 13. Let $\Re_{i}=\left\{\left\langle\left[s_{x_{i}^{-}}, s_{y_{i}^{-}}, s_{z_{i}^{-}}\right],\left[s_{x_{i}^{+}}, s_{y_{i}^{+}}, s_{z_{i}^{+}}\right]\right\rangle ; s_{\varkappa_{i}}, s_{\phi_{i}}, s_{\varphi_{i}}\right\}(i=1, \ldots, n)$ are the family of triangular linguistic cubic fuzzy variables. A triangular linguistic cubic fuzzy Dombi order weighted geometric (TLCFDOWG) operator is a function TLCFDOWG $: \Omega^{n} \rightarrow \Omega$, and is defined as below,

$$
T L C F D O W G_{\Theta}\left(\Re_{1}, \ldots, \Re_{n}\right)=\left(\Re_{\sigma_{(1)}}\right)^{\Theta_{1}} \otimes \ldots \otimes\left(\Re_{\sigma_{(i)}}\right)^{\Theta_{i}} .
$$

Then, the operator TLCFDOWG is called triangular linguistic cubic fuzzy Dombi order weighted geometric operator of dimension $n, \forall i, \Re_{\sigma_{(i-1)}} \geq \Re_{\sigma_{(i)}}\left(\sigma_{(1)}, \ldots, \sigma_{(n)}\right)$ is the permutation of $(1, \ldots, n)$. Also, the weight vector of $\Re_{i}(i=1, \ldots, n)$ is $\Theta=$ $\left(\Theta_{1}, \ldots, \Theta_{n}\right)^{T}$ with $\sum_{i=1}^{n} \Theta_{i}=1$ and $\Theta_{i} \in[0,1]$. Moreover, the TLCFDOWG operator converts to the TLCFDG operator of dimension $n$ if $\varpi=(1 / n, \ldots, 1 / n)^{T}$, which is given as;

$$
T L C F D O W G_{\Theta}\left(\Re_{1}, \ldots, \Re_{n}\right)=1 / n\left(\Re_{1} \otimes \ldots \otimes \Re_{n}\right) .
$$

Definition 14. Let $\Re_{i}=\left\{\left\langle\left[s_{x_{i}^{-}}, s_{y_{i}^{-}}, s_{z_{i}^{-}}\right],\left[s_{x_{i}^{+}}, s_{y_{i}^{+}}, s_{z_{i}^{+}}\right]\right\rangle ; s_{\varkappa_{i}}, s_{\phi_{i}}, s_{\varphi_{i}}\right\}(i=1, \ldots, n)$ are the family of triangular linguistic cubic fuzzy variables. Then, their aggregated value by using the TLCFDOWG operator is also a TLCFV and as;

$$
\begin{aligned}
& \text { (4.18)TLCFDOWG } G_{\Theta}\left(\Re_{1}, \ldots, \Re_{n}\right)=\prod_{i=1}^{n}\left(\Re_{\sigma(i)}\right)^{\Theta_{i}}
\end{aligned}
$$

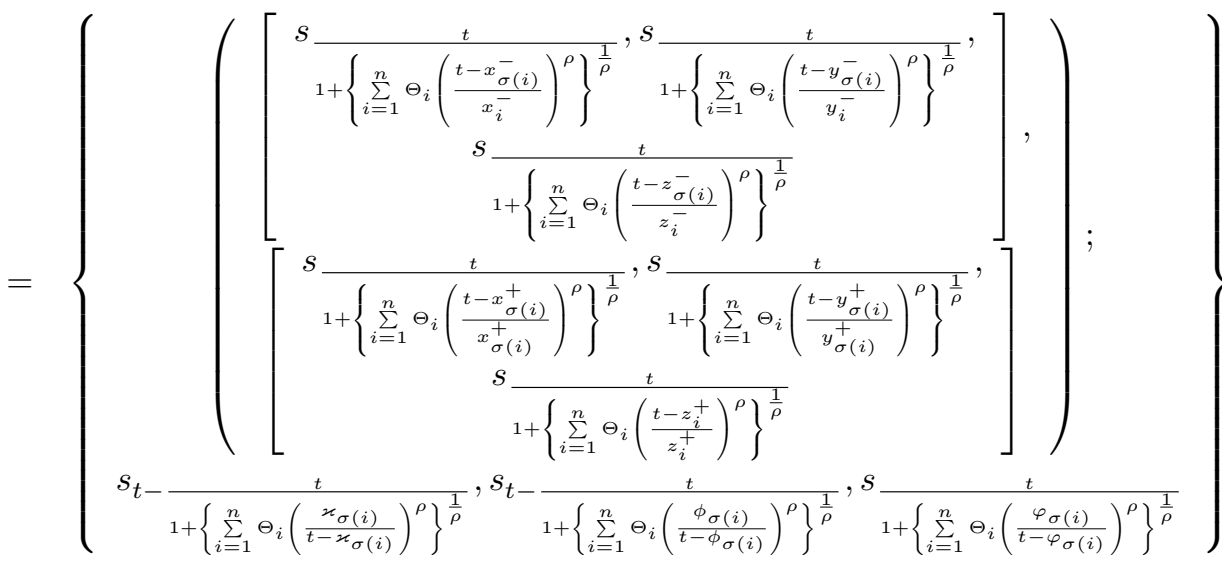

Also, the weighting vector of $\Re_{i}(i=1, \ldots, n)$ is $\Theta=\left(\Theta_{1}, \ldots, \Theta_{n}\right)^{T}$ with $\sum_{i=1}^{n} \Theta_{i}=1$ and $\Theta_{i} \in[0,1]$ the order represented by permutation $\left(\sigma_{(1)}, \ldots, \sigma_{(n)}\right)$ of $(i=1, \ldots, n)$ subject to $\Re_{\sigma_{(i-1)}} \geq \Re_{\sigma_{(i)}}$ for all $(i=1, \ldots, n)$.

Definition 15. Let $\Re_{i}=\left\{\left\langle\left[s_{x_{i}^{-}}, s_{y_{i}^{-}}, s_{z_{i}^{-}}\right],\left[s_{x_{i}^{+}}, s_{y_{i}^{+}}, s_{z_{i}^{+}}\right]\right\rangle ; s_{\varkappa_{i}}, s_{\phi_{i}}, s_{\varphi_{i}}\right\}(i=1, \ldots, n)$ are the family of triangular linguistic cubic fuzzy variables. A triangular linguistic cubic fuzzy Dombi hybrid geometric (TLCFDHG) operator is a function TLCFDHG : $\Omega^{n} \rightarrow \Omega$, with the associated weighting vector $\varpi=\left(\varpi_{1}, \ldots, \varpi_{n}\right)^{T}$ and $\sum_{i=1}^{n} \varpi_{i}=1$ and $\varpi_{i} \in[0,1]$, such that,

$$
T L C F D H G_{\Theta, \varpi}\left(\Re_{1}, \ldots, \Re_{n}\right)=\left(\widetilde{\Re}_{\sigma_{(1)}}\right)^{\Theta_{1}} \otimes \ldots \otimes\left(\widetilde{\Re}_{\sigma_{(i)}}\right)^{\Theta_{i}} .
$$


Here, $\widetilde{\Re}_{\sigma_{(i)}}$ is the $i^{\text {th }}$ biggest value of the weighted triangular linguistic cubic fuzzy values $\Re_{i}$. Also, the weight of $\Re_{i}$ is $\Theta=\left(\Theta_{1}, \ldots, \Theta_{n}\right)^{T}$, with $\sum_{i=1}^{n} \Theta_{i}=1$ and $\Theta_{i} \in$ $[0,1]$, i.e., $\left.\widetilde{\Re}_{i}=\left(\Re_{i}\right)^{n \varpi_{i}}=\left\{\left\langle\left[s_{\widetilde{x}_{i}^{-}}, s_{\widetilde{y}_{i}^{-}}, s_{\widetilde{z}_{i}^{-}}\right],\left[s_{\widetilde{x}_{i}^{+}}, s_{\widetilde{y}_{i}^{+}}, s_{\widetilde{z}_{i}^{+}}\right]\right\rangle ; s_{\widetilde{\varkappa}_{i}}, s_{\widetilde{\phi}_{i}}, s_{\widetilde{\varphi}_{i}}\right]\right\}(i=$ $1, \ldots, n)$. Where, $s_{\widetilde{x}_{i}^{-}}=s \frac{t}{1+\left\{n \varpi_{i}\left(\frac{t-x_{1}^{-}}{x_{1}^{-}}\right)^{\rho}\right\}^{\frac{1}{\rho}}}, s_{\widetilde{y}_{i}^{-}}=s \frac{t}{1+\left\{n \varpi_{i}\left(\frac{t-y_{1}^{-}}{y_{1}^{-}}\right)^{\rho}\right\}^{\frac{1}{\rho}}}, s_{\widetilde{z}_{i}^{-}}=s \frac{t}{1+\left\{n \varpi_{i}\left(\frac{t-z_{1}^{-}}{z_{1}^{-}}\right)^{\rho}\right\}_{t}^{\frac{1}{\rho}}}, s_{\widetilde{x}_{i}^{+}}=$
$\left.s \frac{t}{1+\left\{n \varpi_{i}\left(\frac{t-y_{1}^{+}}{y_{1}^{-}}\right)^{\rho}\right\}^{\frac{1}{\rho}}}, s_{\widetilde{z}_{i}^{+}}=s \frac{s_{\widetilde{\varkappa}_{i}^{-}}}{1+\left\{n \varpi_{i}\left(\frac{t-z_{1}^{+}}{z_{1}^{-}}\right)^{\rho}\right\}^{\frac{1}{\rho}}}, s_{t-\widetilde{x}_{1}^{+}}\right)^{\frac{1}{\rho}} s_{\widetilde{y}_{i}^{+}}=s \frac{s_{\widetilde{\phi}_{i}}}{1+\left\{n \varpi_{i}\left(\frac{\varkappa_{1}}{t-\varkappa_{1}^{-}}\right)^{\rho}\right\}^{\frac{1}{\rho}}}=$

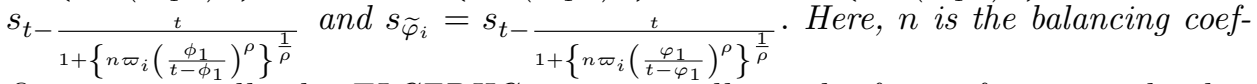
ficient, especially the TLCFDHG operator will get the form of a triangular linguistic cubic fuzzy Dombi geometric (TLCFDHG) operator of dimension $n$ if $\varpi=$ $(1 / n, \ldots, 1 / n)^{T}$.

Theorem 12. Let $\Re_{i}=\left\{\left\langle\left[s_{x_{i}^{-}}, s_{y_{i}^{-}}, s_{z_{i}^{-}}\right],\left[s_{x_{i}^{+}}, s_{y_{i}^{+}}, s_{z_{i}^{+}}\right]\right\rangle ; s_{\varkappa_{i}}, s_{\phi_{i}}, s_{\varphi_{i}}\right\}(i=1, \ldots, n)$ are the family of triangular linguistic cubic fuzzy variables. Then, their aggregated value by utilizing the TLCFDHG operator is also a TLCFV and as;

$$
\begin{aligned}
& \left(4.20 \not C F D H G_{\Theta, \varpi}\left(\Re_{1}, \ldots, \Re_{n}\right)=\prod_{i=1}^{n}\left(\widetilde{\Re}_{\sigma(i)}\right)^{\Theta_{i}}\right.
\end{aligned}
$$

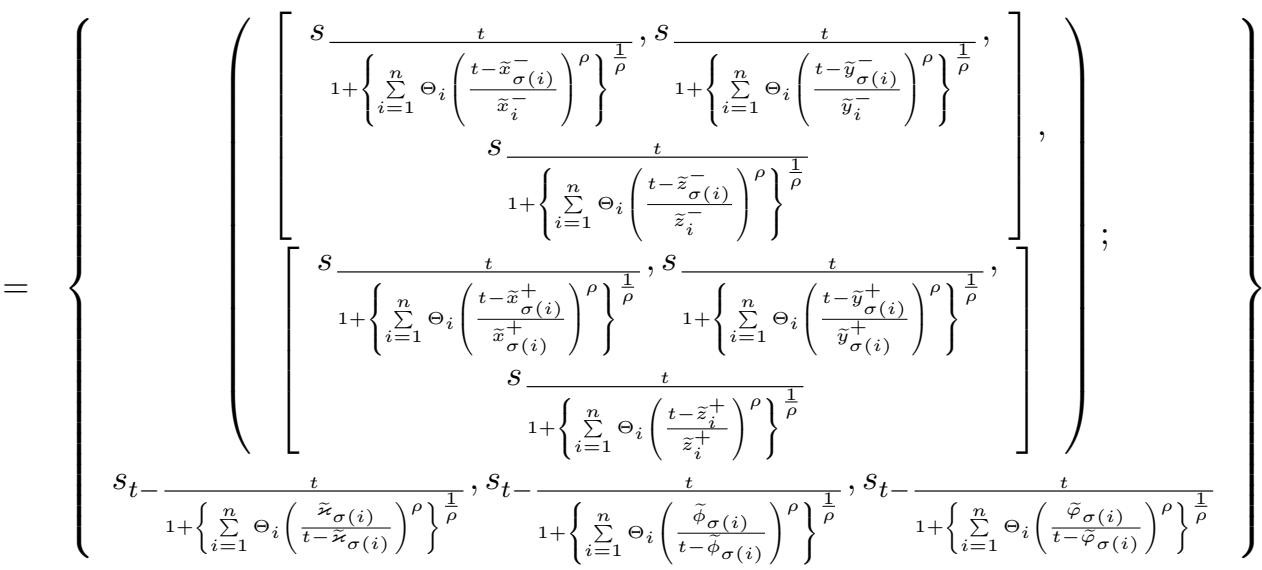

Also, the weighting vector of $\Re_{i}(i=1, \ldots, n)$ is $\Theta=\left(\Theta_{1}, \ldots, \Theta_{n}\right)^{T}$ with $\sum_{i=1}^{n} \Theta_{i}=1$ and $\Theta_{i} \in[0,1]$ the order represented by permutation $\left(\sigma_{(1)}, \ldots, \sigma_{(n)}\right)$ of $(i=1, \ldots, n)$ subject to $\Re_{\sigma_{(i-1)}} \geq \Re_{\sigma_{(i)}}$ for all $(i=1, \ldots, n)$.

\section{Approach for Madm Making Using Triangular Linguistic Cubic FUZZY VARIABLES}

In this section, we use triangular linguistic cubic fuzzy Dombi weighted average and geometric aggregation operators for multi-criteria decision making MCDM problem. Assume that, we have $n$ alternatives $\Im=\left\{\Im_{1}, \ldots, \Im_{n}\right\}$ and $m$ criteria $\mathbb{N}=\left\{\mathbb{N}_{1}, \ldots, \mathbb{N}_{m}\right\}$ to be evaluated with associated weight vector $\Theta=\left(\Theta_{1}, \ldots, \Theta_{n}\right)^{T}$, where $\Theta_{j} \in[0,1]$ and $\sum_{j=1}^{n} \Theta_{j}=1$. To evaluate the performance of the alternative 
$\Im_{i}$ on the basis of criteria $\mathbb{N}_{j}$, these have provided the information about the alternative $\Im_{i}$ satisfying the criteria $\mathbb{N}_{j}$. The rating of alternatives $\Im_{i}$ on criteria $\mathbb{N}_{j}$, given by experts in the form of triangular linguistic cubic fuzzy variables as $\Im_{j}=$ $\left\{\left[s_{x_{j}^{-}}, s_{y_{j}^{-}}, s_{z_{j}^{-}}\right],\left[s_{x_{j}^{+}}, s_{y_{j}^{+}}, s_{z_{j}^{+}}\right] ; s_{\varkappa_{j}}, s_{\phi_{j}}, s_{\varphi_{j}}\right\}(i=1, \ldots n ; j=1, \ldots, m)$. Thus, a MCDM problem can be briefly expressed is an triangular linguistic cubic fuzzy variables decision matrix, $D=\left(\Im_{i j}\right)_{n \times m}=\left\{\left[s_{x_{i j}^{-}}, s_{y_{i j}^{-}}, s_{z_{i j}^{-}}\right],\left[s_{x_{i j}^{+}}, s_{y_{i j}^{+}}, s_{z_{i j}^{+}}\right] ; s_{\varkappa_{i j}}, s_{\phi_{i j}}, s_{\varphi_{i j}}\right\}_{n \times m}(i=$ $1, \ldots, n ; j=1, \ldots, m)$.

Step 1. Make triangular linguistic cubic fuzzy variable decision matrix. $D=$ $\left(\Im_{i j}\right)_{n \times m}=\left\{\left[s_{x_{i j}^{-}}, s_{y_{i j}^{-}}, s_{z_{i j}^{-}}\right],\left[s_{x_{i j}^{+}}, s_{y_{i j}^{+}}, s_{z_{i j}^{+}}\right] ; s_{\varkappa_{i j}}, s_{\phi_{i j}}, s_{\varphi_{i j}}\right\}_{n \times m}(i=1, \ldots, n ; j=$ $1, \ldots, m)$.

Step 2. Usually, the criteria can be classified into two types, benefit criteria and cost criteria. Using the below formula, if the decision matrix has both types of criteria; $D_{i j}=\left\{\begin{array}{c}\Im_{i j} \text {, if the criteria is of benefit type } \\ \Im_{i j}^{c} \text {, if the criteria is of cost type }\end{array}\right\}, \Im_{i j}^{c}$ is the complement of $\Im_{i j}$. Hence, we obtain the normalized triangular linguistic cubic fuzzy variable decision matrix. The normalized triangular linguistic cubic variable decision matrix is denoted by $D^{n}$.

Step 3. Using the proposed aggregation operators to find the TLCFVs $\Im_{i}(i=$ $1, \ldots, n)$ for the alternatives $\Im_{i}(i=1, \ldots, n)$. i.e., the developed operators to stem the collective total preference values $\Im_{i}(i=1, \ldots, n)$ of the alternatives $\Im_{i}$, where $\Theta=\left(\Theta_{1}, \ldots, \Theta_{n}\right)^{T}$ is the weights of the criteria.

Step 4. By the uses of Equ. (2.4), find the scores of the alternatives $\Im_{i}$.

Step 5. Finally, give ranking to the alternatives, and choose the best one.

\section{ExAMPLE}

In the following section, we apply our developed concept of the TLCFVs to a practical problem of purchase selection. We consider the example from the field of the marketing.

6.1. Purchase Satisfaction Problem. Purchase Satisfaction or more specifically post-purchase product satisfaction or post-purchase satisfaction is a marketing term which shows the satisfaction or the pleasure a customer gets after purchasing a specific product of his choice from the market. We make use of the TLCFVs to determine the level of the satisfaction a customer gets after buying a mobile set from the market. More information on purchase satisfaction can be found in [38]. In this example, we use the TLCFVs data. We have three expert as listed in the set:

$$
E=\{\text { Sheraz, Anosha, Mustfa }\}
$$

They want to buy four mobile phone is given as:

$$
\Im=\{\text { Mobile }-1 ; \text { Mobile }-2 ; \text { Mobile }-3 ; \text { Mobile }-4\}
$$

one each from a market. Since, the consumers' purchasing decisions are dependent on their choices, so they purchase mobiles through prioritizing their choices. Each customer is interested in prioritizing the four features in a mobile, in accordance with his/her satisfaction, given in the set,

$$
\mathbb{N}=\{\text { Price; Storage; Camera; Looks }\}
$$


The four features are respectively measured as price in dollar, storage in Gigabytes (GB), camera in pixels and looks as excellent or very good or good or fair with the weight vector are $\Theta=(0.3,0.2,0.1,0.4)^{T}$. The data from the five customers about the four features of the mobiles is collected on four questionnaires [40]. After preliminary statistical analysis like that of Likert Scale [31], the experts decided to give the evaluation value in the way of triangular linguistic cubic fuzzy term and the linguistic evaluation set $\dot{S}=\left\{s_{1}, s_{2}, s_{3}, s_{4}, s_{5}, s_{6}, s_{7}, s_{8}, s_{9}\right\}$ and the associated weights are $\varpi=(0.3,0.2,0.3,0.2)^{T}$ in the decision Table $1,2,3$.

Step 1. The experts have given their decisions in Table 1.

Table 1. The triangular linguistic cubic fuzzy variables decision matrix

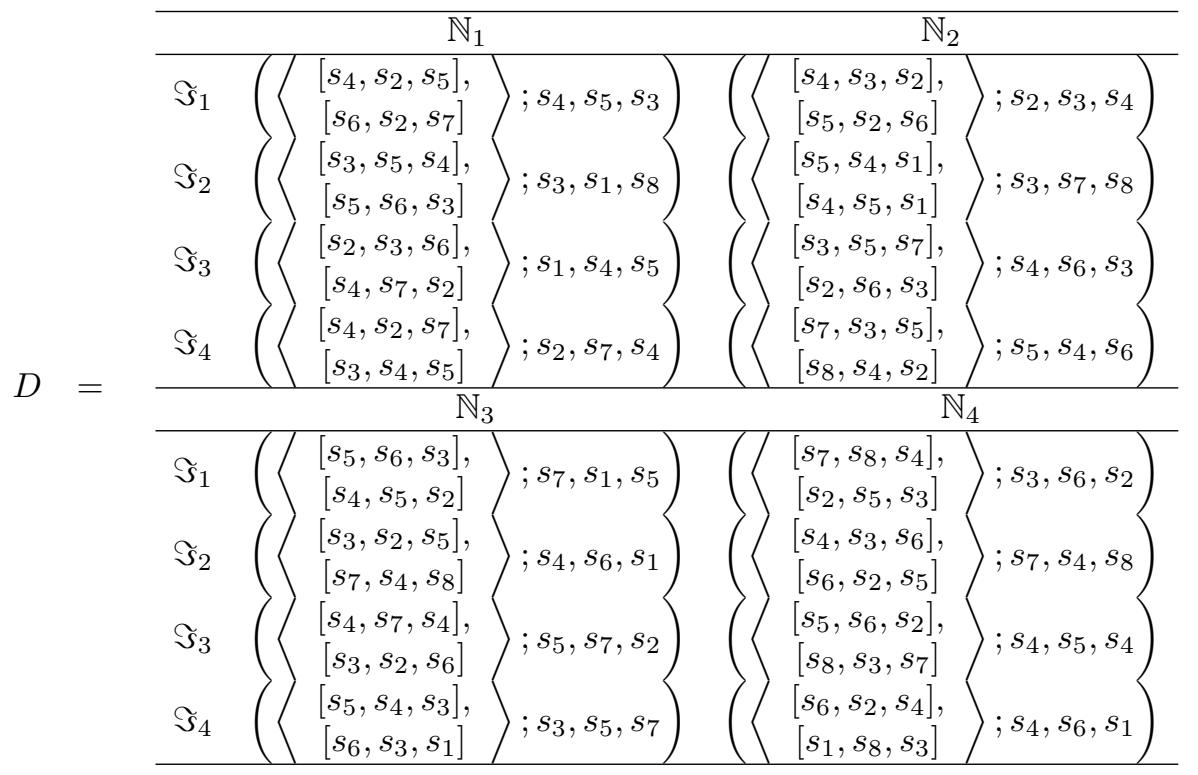

Step 2. As all the criteria have the same type (benifit), so the normalization is not needed.

Using Triangular Linguistic Cubic Fuzzy Dombi Weighted Averaging (TLCFDWA) Operator

Step 3. Using TLCFDWA operator (Equ. 4.2), having $\psi=(0.3,0.2,0.1,0.4)^{T}$ as weight vector, we get the collective TLCFVs for the alternatives $\Im_{i}(i=1, \ldots, 4)$.

$$
\begin{aligned}
& \Im_{1}=\left(\left\langle\left[s_{5.923}, s_{7.037}, s_{4.005}\right],\left[s_{4.597}, s_{3.909}, s_{5.640}\right]\right\rangle ; s_{3.099}, s_{3.409}, s_{2.702}\right) \\
& \Im_{2}=\left(\left\langle\left[s_{3.915}, s_{3.896}, s_{4.890}\right],\left[s_{5.648}, s_{4.597}, s_{5.363}\right]\right\rangle ; s_{4.019}, s_{2.245}, s_{4.705}\right) \\
& \Im_{3}=\left(\left\langle\left[s_{3.902}, s_{5.470}, s_{5.391}\right],\left[s_{7.020}, s_{5.640}, s_{5.769}\right]\right\rangle ; s_{2.127}, s_{4.935}, s_{3.614}\right) \\
& \Im_{4}=\left(\left\langle\left[s_{5.858}, s_{2.478}, s_{5.629}\right],\left[s_{6.000}, s_{7.064}, s_{3.527}\right]\right\rangle ; s_{3.092}, s_{5.570}, s_{1.913}\right)
\end{aligned}
$$

Step 4. Using Equ. (2.4), compute the scores $S c\left(\Im_{i}\right)$ of $\Im_{i}(i=1, \ldots, 4)$ as follows;

$$
S c\left(\Im_{1}\right)=0.3478, S c\left(\Im_{2}\right)=0.3131, S c\left(\Im_{3}\right)=0.3305, S c\left(\Im_{4}\right)=0.3243
$$


Step 5. Ranking of the alternatives according to the scores are $\Im_{1}>\Im_{3}>$ $\Im_{4}>\Im_{2}$. Thus, $\Im_{1}$ is the best choice.

Table 2. Ranking of the alternatives using different values of parameters $\rho$ based on TLCFDWA operator

\begin{tabular}{lllllc}
\hline$\rho$ & $S c\left(\Im_{1}\right)$ & $S c\left(\Im_{2}\right)$ & $S c\left(\Im_{3}\right)$ & $S c\left(\Im_{4}\right)$ & Ranking \\
\hline 1 & 0.3478 & 0.3131 & 0.3305 & 0.3243 & $\Im_{1}>\Im_{3}>\Im_{4}>\Im_{2}$ \\
5 & 0.4273 & 0.4134 & 0.4036 & 0.4011 & $\Im_{1}>\Im_{3}>\Im_{4}>\Im_{2}$ \\
10 & 0.4423 & 0.4215 & 0.4322 & 0.4234 & $\Im_{1}>\Im_{3}>\Im_{4}>\Im_{2}$ \\
25 & 0.4512 & 0.4462 & 0.4356 & 0.4359 & $\Im_{1}>\Im_{3}>\Im_{4}>\Im_{2}$ \\
50 & 0.4536 & 0.4402 & 0.4501 & 0.4416 & $\Im_{1}>\Im_{3}>\Im_{4}>\Im_{2}$ \\
\hline
\end{tabular}

Using Triangular Linguistic Cubic Fuzzy Dombi Ordered Weighted Averaging (TLCFDOWA) Operator

Step 3. Using TLCFDOWA operator (Equ. 4.8), having $\psi=(0.3,0.2,0.1,0.4)^{T}$ as weight vector, we get the collective TLCFVs for the alternatives $\Im_{i}(i=1, \ldots, 4)$.

$$
\begin{aligned}
& \Im_{1}=\left(\left\langle\left[s_{5.363}, s_{6.375}, s_{2.683}\right],\left[s_{4.493}, s_{3.909}, s_{5.146}\right]\right\rangle ; s_{2.989}, s_{2.054}, s_{3.409}\right) \\
& \Im_{2}=\left(\left\langle\left[s_{4.161}, s_{3.480}, s_{4.275}\right],\left[s_{5.891}, s_{4.493}, s_{6.600}\right]\right\rangle ; s_{3.700}, s_{3.888}, s_{2.580}\right) \\
& \Im_{3}=\left(\left\langle\left[s_{3.703}, s_{5.571}, s_{5.243}\right],\left[s_{6.661}, s_{5.799}, s_{5.557}\right]\right\rangle ; s_{1.851}, s_{4.872}, s_{3.468}\right) \\
& \Im_{4}=\left(\left\langle\left[s_{6.022}, s_{3.214}, s_{4.683}\right],\left[s_{6.894}, s_{6.115}, s_{2.385}\right]\right\rangle ; s_{3.409}, s_{4.904}, s_{3.010}\right)
\end{aligned}
$$

Step 4. Using Equ. (2.4), compute the scores $S c\left(\Im_{i}\right)$ of $\Im_{i}(i=1, \ldots, 4)$ as follows;

$$
S c\left(\Im_{1}\right)=0.3247, S c\left(\Im_{2}\right)=0.2914, S c\left(\Im_{3}\right)=0.3222, S c\left(\Im_{4}\right)=0.3062
$$

Step 5. Ranking of the alternatives according to the scores are $\Im_{1}>\Im_{3}>$ $\Im_{4}>\Im_{2}$. Thus, $\Im_{3}$ is the best choice.

Table 3. Ranking of the alternatives for different parameters $\rho$ based on TLCFDOWA operator

\begin{tabular}{lllllc}
\hline$\rho$ & $S c\left(\Im_{1}\right)$ & $S c\left(\Im_{2}\right)$ & $S c\left(\Im_{3}\right)$ & $S c\left(\Im_{4}\right)$ & Ranking \\
\hline 1 & 0.3247 & 0.2914 & 0.3222 & 0.3062 & $\Im_{1}>\Im_{3}>\Im_{4}>\Im_{2}$ \\
5 & 0.3770 & 0.3499 & 0.3691 & 0.3581 & $\Im_{1}>\Im_{3}>\Im_{4}>\Im_{2}$ \\
10 & 0.3952 & 0.3396 & 0.3502 & 0.3480 & $\Im_{1}>\Im_{3}>\Im_{4}>\Im_{2}$ \\
25 & 0.4468 & 0.4101 & 0.4399 & 0.4177 & $\Im_{1}>\Im_{3}>\Im_{4}>\Im_{2}$ \\
50 & 0.5287 & 0.4683 & 0.5021 & 0.4971 & $\Im_{1}>\Im_{3}>\Im_{4}>\Im_{2}$ \\
\hline
\end{tabular}

Using Triangular Linguistic Cubic Fuzzy Dombi Hybrid Averaging (TLCFDHA) Operator

Step 3. Using TLCFDHA operator (Equ. 4.10), having $\psi=(0.3,0.2,0.1,0.4)^{T}$ as weight vector, we get the collective TLCFVs for the alternatives $\Im_{i}(i=1, \ldots, 4)$.

$$
\begin{aligned}
& \Im_{1}=\left(\left\langle\left[s_{4.433}, s_{3.972}, s_{5.383}\right],\left[s_{3.443}, s_{4.849}, s_{3.456}\right]\right\rangle ; s_{4.327}, s_{4.794}, s_{2.909}\right) \\
& \Im_{2}=\left(\left\langle\left[s_{3.129}, s_{2.498}, s_{3.259}\right],\left[s_{3.119}, s_{5.456}, s_{4.730}\right]\right\rangle ; s_{5.760}, s_{4.889}, s_{3.507}\right) \\
& \Im_{3}=\left(\left\langle\left[s_{5.366}, s_{4.143}, s_{3.329}\right],\left[s_{4.647}, s_{4.734}, s_{3.538}\right]\right\rangle ; s_{3.189}, s_{3.874}, s_{4.416}\right) \\
& \Im_{4}=\left(\left\langle\left[s_{3.290}, s_{2.244}, s_{5.635}\right],\left[s_{4.875}, s_{4.531}, s_{2.383}\right]\right\rangle ; s_{5.494}, s_{3.963}, s_{4.175}\right)
\end{aligned}
$$

Step 4. Using Equ. (2.4), compute the scores $S c\left(\Im_{i}\right)$ of $\Im_{i}(i=1, \ldots, 4)$ as follows;

$$
S c\left(\Im_{1}\right)=0.2756, S c\left(\Im_{2}\right)=0.2413, S c\left(\Im_{3}\right)=0.2549, S c\left(\Im_{4}\right)=0.2506
$$


Step 5. Ranking of the alternatives according to the scores are $\Im_{3}>\Im_{1}>$ $\Im_{4}>\Im_{2}$. Thus, $\Im_{3}$ is the best choice.

Table 4. Ranking of the alternatives using different values of parameter $\rho$ based on TLCFDHA operator

\begin{tabular}{lllllc}
\hline$\rho$ & $S c\left(\Im_{1}\right)$ & $S c\left(\Im_{2}\right)$ & $S c\left(\Im_{3}\right)$ & $S c\left(\Im_{4}\right)$ & Ranking \\
\hline 1 & 0.2756 & 0.2413 & 0.2549 & 0.2506 & $\Im_{3}>\Im_{1}>\Im_{4}>\Im_{2}$ \\
5 & 0.3276 & 0.2891 & 0.3095 & 0.2983 & $\Im_{3}>\Im_{1}>\Im_{4}>\Im_{2}$ \\
10 & 0.3658 & 0.3133 & 0.3323 & 0.3389 & $\Im_{3}>\Im_{1}>\Im_{4}>\Im_{2}$ \\
25 & 0.4068 & 0.3717 & 0.3998 & 0.4391 & $\Im_{3}>\Im_{1}>\Im_{4}>\Im_{2}$ \\
50 & 0.4785 & 0.4189 & 0.4528 & 0.4363 & $\Im_{3}>\Im_{1}>\Im_{4}>\Im_{2}$ \\
\hline
\end{tabular}

Using Triangular Linguistic Cubic Fuzzy Dombi Weighted Geometric (TLCFDWG) Operator

Step 3. Using TLCFDWG operator (Equ. 4.12), having $\psi=(0.3,0.2,0.1,0.4)^{T}$ as weight vector, we get the collective TLCFVs for the alternatives $\Im_{i}(i=1, \ldots, 4)$.

$$
\begin{aligned}
& \Im_{1}=\left(\left\langle\left[s_{4.947}, s_{3.529}, s_{3.409}\right],\left[s_{3.174}, s_{2.857}, s_{3.853}\right]\right\rangle ; s_{4.127}, s_{5.065}, s_{3.190}\right) \\
& \Im_{2}=\left(\left\langle\left[s_{3.658}, s_{3.409}, s_{2.682}\right],\left[s_{5.236}, s_{3.174}, s_{2.547}\right]\right\rangle ; s_{5.703}, s_{4.695}, s_{7.904}\right) \\
& \Im_{3}=\left(\left\langle\left[s_{3.108}, s_{4.525}, s_{3.294}\right],\left[s_{3.870}, s_{3.853}, s_{3.442}\right]\right\rangle ; s_{3.520}, s_{5.385}, s_{4.064}\right) \\
& \Im_{4}=\left(\left\langle\left[s_{5.256}, s_{3.076}, s_{4.625}\right],\left[s_{1.846}, s_{4.800}, s_{2.542}\right]\right\rangle ; s_{3.723}, s_{6.129}, s_{4.588}\right)
\end{aligned}
$$

Step 4. Using Equ. (2.4), compute the scores $S c\left(\Im_{i}\right)$ of $\Im_{i}(i=1, \ldots, 4)$ as follows;

$$
S c\left(\Im_{1}\right)=0.2176, S c\left(\Im_{2}\right)=0.1705, S c\left(\Im_{3}\right)=0.1995, S c\left(\Im_{4}\right)=0.1871
$$

Step 5. Ranking of the alternatives according to the scores are $\Im_{1}>\Im_{3}>$ $\Im_{4}>\Im_{2}$. Thus, $\Im_{1}$ is the best choice.

Table 5. Ranking of the alternatives using different values of parameter $\rho$ based on TLCFDWG operator

\begin{tabular}{lllllc}
\hline$\rho$ & $S c\left(\Im_{1}\right)$ & $S c\left(\Im_{2}\right)$ & $S c\left(\Im_{3}\right)$ & $S c\left(\Im_{4}\right)$ & Ranking \\
\hline 1 & 0.2176 & 0.1705 & 0.1995 & 0.1871 & $\Im_{1}>\Im_{3}>\Im_{4}>\Im_{2}$ \\
5 & 0.1598 & 0.0987 & 0.1139 & 0.1073 & $\Im_{1}>\Im_{3}>\Im_{4}>\Im_{2}$ \\
10 & 0.1427 & 0.0802 & 0.0932 & 0.0854 & $\Im_{1}>\Im_{3}>\Im_{4}>\Im_{2}$ \\
25 & 0.1310 & 0.0691 & 0.0815 & 0.0711 & $\Im_{1}>\Im_{3}>\Im_{4}>\Im_{2}$ \\
50 & 0.1327 & 0.0653 & 0.0858 & 0.0663 & $\Im_{1}>\Im_{3}>\Im_{4}>\Im_{2}$ \\
\hline
\end{tabular}

Using Triangular Linguistic Cubic Fuzzy Dombi Ordered Weighted Geometric (TLCFDOWG) Operator

Step 3. Using TLCFDOWG operator (Equ. 4.18), having $\psi=(0.3,0.2,0.1,0.4)^{T}$ as weight vector, we get the collective TLCFVs for the alternatives $\Im_{i}(i=1, \ldots, 4)$.

$$
\begin{aligned}
& \Im_{1}=\left(\left\langle\left[s_{4.682}, s_{3.870}, s_{2.702}\right],\left[s_{3.680}, s_{2.857}, s_{3.360}\right]\right\rangle ; s_{5.160}, s_{3.893}, s_{4.005}\right) \\
& \Im_{2}=\left(\left\langle\left[s_{3.797}, s_{2.971}, s_{2.936}\right],\left[s_{5.097}, s_{3.680}, s_{1.957}\right]\right\rangle ; s_{4.890}, s_{6.207}, s_{7.644}\right) \\
& \Im_{3}=\left(\left\langle\left[s_{2.912}, s_{4.312}, s_{3.559}\right],\left[s_{3.934}, s_{3.439}, s_{3.231}\right]\right\rangle ; s_{3.444}, s_{5.243}, s_{4.127}\right) \\
& \Im_{4}=\left(\left\langle\left[s_{5.519}, s_{3.016}, s_{3.882}\right],\left[s_{2.962}, s_{3.871}, s_{1.571}\right]\right\rangle ; s_{3.896}, s_{5.385}, s_{6.101}\right)
\end{aligned}
$$

Step 4. Using Equ. (2.4), compute the scores $S c\left(\Im_{i}\right)$ of $\Im_{i}(i=1, \ldots, 4)$ as follows;

$$
S c\left(\Im_{1}\right)=0.2274, S c\left(\Im_{2}\right)=0.1417, S c\left(\Im_{3}\right)=0.1994, S c\left(\Im_{4}\right)=0.1632
$$


Step 5. Ranking of the alternatives according to the scores are $\Im_{1}>\Im_{3}>$ $\Im_{4}>\Im_{2}$. Thus, $\Im_{1}$ is the best choice.

Table 6. Ranking of alternatives using different values of parameter $\rho$ based on TLCFDOWG operator

\begin{tabular}{lllllc}
\hline$\rho$ & $S c\left(\Im_{1}\right)$ & $S c\left(\Im_{2}\right)$ & $S c\left(\Im_{3}\right)$ & $S c\left(\Im_{4}\right)$ & Ranking \\
\hline 1 & 0.2274 & 0.1417 & 0.1994 & 0.1632 & $\Im_{1}>\Im_{3}>\Im_{4}>\Im_{2}$ \\
5 & 0.1624 & 0.0954 & 0.1095 & 0.0983 & $\Im_{1}>\Im_{3}>\Im_{4}>\Im_{2}$ \\
10 & 0.1433 & 0.0810 & 0.0913 & 0.0819 & $\Im_{1}>\Im_{3}>\Im_{4}>\Im_{2}$ \\
25 & 0.1310 & 0.0848 & 0.0807 & 0.0694 & $\Im_{1}>\Im_{3}>\Im_{2}>\Im_{4}$ \\
50 & 0.1192 & 0.0683 & 0.0774 & 0.0701 & $\Im_{1}>\Im_{3}>\Im_{4}>\Im_{2}$ \\
\hline
\end{tabular}

\section{Using Triangular Linguistic Cubic Fuzzy Dombi Hybrid Geometric (TLCFDHG) Operator \\ Step 3. Using TLCFDHG operator (Equ. 4.20), having $\psi=(0.3,0.2,0.1,0.4)^{T}$} as weight vector, we get the collective TLCFVs for the alternatives $\Im_{i}(i=1, \ldots, 4)$.

$$
\begin{aligned}
& \Im_{1}=\left(\left\langle\left[s_{2.629}, s_{4.849}, s_{3.782}\right],\left[s_{4.268}, s_{3.847}, s_{5.436}\right]\right\rangle ; s_{2.184}, s_{4.862}, s_{3.105}\right) \\
& \Im_{2}=\left(\left\langle\left[s_{4.379}, s_{4.933}, s_{3.948}\right],\left[s_{3.917}, s_{3.839}, s_{2.979}\right]\right\rangle ; s_{2.806}, s_{5.273}, s_{6.645}\right) \\
& \Im_{3}=\left(\left\langle\left[s_{4.123}, s_{3.328}, s_{2.599}\right],\left[s_{3.345}, s_{4.149}, s_{2.207}\right]\right\rangle ; s_{5.549}, s_{3.263}, s_{5.142}\right) \\
& \Im_{4}=\left(\left\langle\left[s_{3.359}, s_{4.156}, s_{2.855}\right],\left[s_{3.621}, s_{2.189}, s_{4.547}\right]\right\rangle ; s_{3.946}, s_{4.341}, s_{3.613}\right)
\end{aligned}
$$

Step 4. Using Equ. (2.4), compute the scores $S c\left(\Im_{i}\right)$ of $\Im_{i}(i=1, \ldots, 4)$ as follows;

$$
S c\left(\Im_{1}\right)=0.2399, S c\left(\Im_{2}\right)=0.1841, S c\left(\Im_{3}\right)=0.2083, S c\left(\Im_{4}\right)=0.2065
$$

Step 5. Ranking of the alternatives according to the scores are $\Im_{1}>\Im_{3}>$ $\Im_{4}>\Im_{2}$. Thus, $\Im_{1}$ is the best choice.

Table 7. Ranking of the alternatives using different values of parameter $\rho$ based on TLCFDHG operator

\begin{tabular}{lllllc}
\hline$\rho$ & $S c\left(\Im_{1}\right)$ & $S c\left(\Im_{2}\right)$ & $S c\left(\Im_{3}\right)$ & $S c\left(\Im_{4}\right)$ & Ranking \\
\hline 1 & 0.2399 & 0.1841 & 0.2083 & 0.2065 & $\Im_{1}>\Im_{3}>\Im_{4}>\Im_{2}$ \\
5 & 0.1929 & 0.1354 & 0.1692 & 0.1443 & $\Im_{1}>\Im_{3}>\Im_{4}>\Im_{2}$ \\
10 & 0.1639 & 0.0938 & 0.1173 & 0.1062 & $\Im_{1}>\Im_{3}>\Im_{4}>\Im_{2}$ \\
25 & 0.1418 & 0.0888 & 0.0971 & 0.0897 & $\Im_{1}>\Im_{3}>\Im_{2}>\Im_{4}$ \\
50 & 0.1224 & 0.0712 & 0.0826 & 0.0784 & $\Im_{1}>\Im_{3}>\Im_{4}>\Im_{2}$ \\
\hline
\end{tabular}

6.2. Comparative analysis. Within this portion, we compare the result of the defined MCDM method with some of the current methods like as intuitionistic cubic fuzzy set (ICFS), triangular cubic fuzzy numbers (TCFNs), triangular cubic linguistic hesitant fuzzy set (TCLHFS) and triangular cubic linguistic uncertain fuzzy set (TCLUFSs). For it, first the priorities considered by the experts are translated into IFS, TCFNs, TCLHFS and TCLUFSs. On the basis of this environment, we applied the current methods, and their results are given as; 
Table 8. Comparison with some existing methods

\begin{tabular}{|l|cccc|c|}
\hline \multirow{2}{*}{ Approach } & \multicolumn{4}{|c|}{ Score values } & \multirow{2}{*}{ Ranking } \\
& $\Im_{1}$ & $\Im_{2}$ & $\Im_{3}$ & $\Im_{4}$ & \\
\hline Abdullah et al. [5] & 0.482 & 0.354 & 0.432 & 0.395 & $\Im_{1}>\Im_{3}>\Im_{4}>\Im_{2}$ \\
\hline Abdullah et al. [6] & 0.190 & 0.143 & 0.164 & 0.136 & $\Im_{1}>\Im_{3}>\Im_{2}>\Im_{4}$ \\
\hline Fahmi et al. [11] & 0.803 & 0.768 & 0.713 & 0.694 & $\Im_{1}>\Im_{3}>\Im_{4}>\Im_{2}$ \\
\hline Amin et al. [2] & 0.397 & 0.321 & 0.346 & 0.368 & $\Im_{1}>\Im_{4}>\Im_{3}>\Im_{2}$ \\
\hline Amin et al. [3] & 0.638 & 0.595 & 0.542 & 0.562 & $\Im_{1}>\Im_{2}>\Im_{4}>\Im_{3}$ \\
\hline
\end{tabular}

We give some characteristics comparison of our proposed method and the aforementioned methods, which are listed in Table 8. The method proposed by Abdullah et al. $[5,6]$ adopts ICFNs to aggregate the uncertain information using averaging and geometric operators only by quantitative aspects. On the other hand, the method described by the author in Fahmi et al. [11] represent the wider range of the information in terms of the TCFN. But their approach is also limited to only quantitative aspects and does not apply the linguistic information. Apart from these, the method proposed by Amin et al. [2,3] adopts TCLHFSs and TCLUFSs to describe the uncertainties in the data as a crisp number. However, in the present study, we proposed the TLCFSs to describe the uncertainties in terms of trainguler linguistic interval pairs of the membership degree and nonmembership degree which can easily express the information in a more semantics and concise way and hence can reduce the information loss.

\section{Conclusion}

Triangular linguistic cubic fuzzy set is an effective tool to express the complex cognitive information in MCDM problems. However, the current research results still have many limitations for the MCDA problem of the fuzzy set analysis of exploiting TLCFVs. Specifically, the operational rules and comparison rules of TLCFVs are not yet complete.

In this article, we developed the notion of triangular linguistic cubic fuzzy variable, also we have described their score function for the comparison of triangular linguistic cubic fuzzy variables. Some triangular linguistic cubic fuzzy variable operational laws have been developed using Dombi t-norm and conorm operation. Also, developed a series of triangular linguistic cubic fuzzy variable aggregation operators. i.e., triangular linguistic cubic fuzzy Dombi weighted average and geometric operators under the triangular linguistic cubic fuzzy variable information. Also, discussed some of its properties like idempotency, boundary, monotonicity and showed a relation between these developed operators. The key feature of these operators is that they find details on the relationship between the aggregated TLCFVs. To demonstrate the efficacy of these operators, we develop a multi-criteria decision making algorithm with triangular linguistic cubic fuzzy information. A particular example shows that the suggested operators provide an alternative way of resolving the decision-making process more effectively. Finally, we got some comparison with current operators to show the validity, practicality and efficacy of the new methodology. 
We will further apply these techniques on different applications like as machine learning, network selection, medical diagnosis, pattern recognitions, image processing, communication problems, clustering problems, computer science and other decision making problems in our future work.

Conflicts of Interest: The authors declare that they have no conflicts of interest.

\section{REFERENCES}

[1] Atanasov, K. T., 1986. Intuitionistic fuzzy sets Fuzzy sets and systems.

[2] Amin, F., Fahmi, A., Abdullah, S., Ali, A, and Ghani, F., 2018, Triangular cubic linguistic hesitant fuzzy aggregation operators and their application in group decision making, Journal of Intelligent and Fuzzy System, 34, 2401-2416.

[3] Amin, F., Fahmi, A. and Aslam, M., 2020. Approaches to multiple attribute group decision making based on triangular cubic linguistic uncertain fuzzy aggregation operators. Soft Computing, 24(15), pp.11511-11533.

[4] Alhazaymeh, K., Gulistan, M., Khan, M. and Kadry, S., 2019. Neutrosophic cubic einstein hybrid geometric aggregation operators with application in prioritization using multiple attribute decision-making method. Mathematics, 7(4), p.346.

[5] Abdullah, S., 2020. Multicriteria group decision-making for supplier selection based on intuitionistic cubic fuzzy aggregation operators. International Journal of Fuzzy Systems, pp.1-14.

[6] Abdullah, S. and Aslam, M., 2020. New multicriteria group decision support systems for small hydropower plant locations selection based on intuitionistic cubic fuzzy aggregation information. International Journal of Intelligent Systems, 35(6), pp.983-1020.

[7] Bordogna, G., Fedrizzi, M. and Pasi, G., 1997. A linguistic modeling of consensus in group decision making based on OWA operators. IEEE Transactions on Systems, Man, and Cybernetics-Part A: Systems and Humans, 27(1), pp.126-133.

[8] Chen, Z., Liu, P. and Pei, Z., 2015. An approach to multiple attribute group decision making based on linguistic intuitionistic fuzzy numbers. International Journal of Computational Intelligence Systems, 8(4), pp.747-760.

[9] Chen, J, and Ye, J., (2017), Some single-valued neutrosophic Dombi weighted aggregation operators for multiple attribute decision-making, Symmetry, 9, 82.

[10] Dombi, J., (1982), A general class of fuzzy operators, the De-Morgan class of fuzzy operators and fuzziness measures induced by fuzzy operators, Fuzzy Sets Syst, 8, 149-163.

[11] Fahmi, A., Abdullah, S., Amin, F., Siddque, N. and Ali, A., 2017, Aggregation operators on triangular cubic fuzzy numbers and its application to Multi-criteria decision making problems, Journal of Intelligent and Fuzzy System, 33, 3323-3337.

[12] Fahmi, A., Abdullah, S., Amin, F, and Ali, A. 2018, Weighted average rating (war) method for solving group decision making problem using triangular cubic fuzzy hybrid aggregation, Punjab University Journal of Mathematics 50(1), 23-34.

[13] Fahmi, A., Amin, F., Abdullah, S. and Ali, A., 2018, Cubic fuzzy Einstein aggregation operators and its application to decision-making, International Journal of Systems Science, $1-13$.

[14] Fu, J., Ye, J. and Cui, W., 2018. An evaluation method of risk grades for prostate cancer using similarity measure of cubic hesitant fuzzy sets. Journal of biomedical informatics, 87 , pp.131-137.

[15] Fahmi, A., Abdullah, S, and Amin, F., 2019, Cubic uncertain linguistic powered Einstein aggregation operators and their application to multi-attribute group decision making, Mathematical Sciences, 13, 129-152.

[16] Fahmi, A., Abdullah, S., Amin, F, and Ali, A., 2019, Precursor selection for sol-gel synthesis of titanium carbide nanopowders by a new cubic fuzzy multi-attribute group decision-making model, Journal of Intelligent Systems. 15(1), 145-167.

[17] Fahmi, A., Abdullah, S., Amin, F, and Khan, M.S.A., 2019, Trapezoidal cubic fuzzy number Einstein hybrid weighted averaging operators and its application to decision making, Soft Comput, 23, 5753-5783. 
[18] Garg, H. and Kumar, K., 2018. Group decision making approach based on possibility degree measure under linguistic interval-valued intuitionistic fuzzy set environment. Journal of Industrial \& Management Optimization, pp.466-482.

[19] Herrera, F., Herrera-Viedma, E. and Martinez, L., 2000. A fusion approach for managing multi-granularity linguistic term sets in decision making. Fuzzy sets and systems, 114(1), pp.43-5.

[20] Herrera, F. and Martinez, L., 2001. A model based on linguistic 2-tuples for dealing with multigranular hierarchical linguistic contexts in multi-expert decision-making. IEEE Transactions on Systems, Man, and Cybernetics, Part B (Cybernetics), 31(2), pp.227-2348.

[21] He, X., 2017, Typhoon disaster assessment based on Dombi hesitant fuzzy information aggregation operators, Nat. Hazards, 90, 1153-1175.

[22] Jun, Y.B., Kim, C.S, and Yang, Ki.O., 2011, Cubic sets, Annals of Fuzzy Mathematics and Informatics, 4(1), 83-98.

[23] Jun, Y.B., Smarandache, F. and Kim, C.S., 2017. Neutrosophic cubic sets. New mathematics and natural computation, 13(01), pp.41-54.

[24] Jun, Y.B., Song, S.Z. and Kim, S.J., 2018. Cubic interval-valued intuitionistic fuzzy sets and their application in BCK/BCI-algebras. Axioms, 7(1), p.7.

[25] Jana, C., Pal, M, and Wang, J.Q., 2018, Bipolar fuzzy Dombi aggregation operators and its application in multiple-attribute decision-making process, J. Ambient. Intell. Humaniz. Comput, 1-17.

[26] Jana, C., Senapati, T., Pal, M. and Saeid, A.B., 2019. Different types of cubic ideals in BCI-algebras based on fuzzy points. Afrika Matematika, pp.1-15.

[27] Jana, C., Senapati, T., Pal, M, and Yager, R.R., 2019, Picture fuzzy Dombi aggregation operators: Application to MADM process, Appl. Soft Comput, 74, 99-109.

[28] Khan, M., Abdullah, S., Zeb, A, and Majid, A., 2016, CUBIC AGGREGATION OPERATORS, International Journal of Computer Science and Information Security, 14, 670.

[29] Khan, A. A., Ashraf, S., Abdullah, S., Qiyas, M., Luo, J, and Khan,S., 2019, Pythagorean Fuzzy Dombi Aggregation Operators and Their Application in Decision Support System, Symmetry, 11, 383.

[30] Liao, X., Li, Y. and Lu, B., 2007. A model for selecting an ERP system based on linguistic information processing. Information Systems, 32(7), pp.1005-1017.

[31] Li, Q., 2013. A novel Likert scale based on fuzzy sets theory. Expert Systems with Applications, 40(5), pp.1609-1618.

[32] Liu, P., Liu, J, and Chen, S.M., 2018, Some intuitionistic fuzzy Dombi Bonferroni mean operators and their application to multi-attribute group decision-making, J. Oper. Res. Soc, $69,1-24$

[33] Lu, X, and Ye, J., 2018, Dombi aggregation operators of linguistic cubic variables for multiple attribute decision-making, Information, 9, 188.

[34] Martı, L. and Herrera, F., 2012. An overview on the 2-tuple linguistic model for computing with words in decision making: Extensions, applications and challenges. Information Sciences, 207, pp.1-18.

[35] Mahmood, T., Mehmood, F, and Khan, Q., 2016, Cubic hesitant fuzzy set and their application to multi-criteria decision making, Int. J. Algebra Stat, 5, 19-51.

[36] Ma, X.L., Zhan, J., Khan, M., Gulistan, M. and Yaqoob, N., 2018. Generalized cubic relations in $\mathrm{H}$ v-LA-semigroups. Journal of Discrete Mathematical Sciences and Cryptography, 21(3), pp.607-630.

[37] Peng, H.G., Wang, J.Q. and Cheng, P.F., 2018. A linguistic intuitionistic multi-criteria decision-making method based on the Frank Heronian mean operator and its application in evaluating coal mine safety. International Journal of Machine Learning and Cybernetics, 9(6), pp.1053-1068.

[38] Richins, M.L. and Bloch, P.H., 1991. Post-purchase product satisfaction: Incorporating the effects of involvement and time. Journal of Business Research, 23(2), pp.145-158.

[39] Rodriguez, R.M., Martinez, L. and Herrera, F., 2012. Hesitant fuzzy linguistic term sets for decision making. IEEE Transactions on Fuzzy Systems, 20(1), pp.109-119.

[40] Rowley, J., 2014. Designing and using research questionnaires. Management Research Review.

[41] Rashid, S., Yaqoob, N., Akram, M. and Gulistan, M., 2018. Cubic graphs with application. International Journal of Analysis and Applications, 16(5), pp.733-750. 
[42] Rashid, S., Gulistan, M., Jun, Y.B., Khan, S. and Kadry, S., 2019. N-Cubic sets and aggregation operators. Journal of Intelligent \& Fuzzy Systems, 37(4), pp.5009-5023.

[43] Shi, L. and Ye, J., 2018. Dombi aggregation operators of neutrosophic cubic sets for multiple attribute decision-making. Algorithms, 11, 29.

[44] Shakeel, M., 2018. Cubic averaging aggregation operators with multiple attributes group decision making problem. Journal of Biostatistics and Biometric Applications, 3(1), pp.1119.

[45] Senapati, T., Jun, Y.B. and Shum, K.P., 2018. Cubic set structure applied in UP-algebras. Discrete Mathematics, Algorithms and Applications, 10(04), p.1850049.

[46] Senapati, T., Jun, Y.B., Muhiuddin, G. and Shum, K.P., 2019. Cubic intuitionistic structures applied to ideals of BCI-algebras. Analele Universitatii" Ovidius" Constanta-Seria Matematica, 27(2), pp.213-232

[47] Senapati, T., Jun, Y.B. and Shum, K.P., 2020. Cubic intuitionistic structure of KU-algebras. Afrika Matematika, 31(2), pp.237-248.

[48] Wang, J.Q. and Li, J.J., 2009. The multi-criteria group decision making method based on multi-granularity intuitionistic two semantics. Science \& Technology Information, 33(1), pp.8-

[49] $\mathrm{Wu}, \mathrm{Z}$. and $\mathrm{Xu}$, J., 2015. Possibility distribution-based approach for MAGDM with hesitant fuzzy linguistic information. IEEE transactions on cybernetics, 46(3), pp.694-705.

[50] Wang, P., Xu, X., Wang, J. and Cai, C., 2017. Interval-valued intuitionistic linguistic multicriteria group decision-making method based on the interval 2-tuple linguistic information. Journal of Intelligent \& Fuzzy Systems, 33(2), pp.985-994.

[51] Xu, Z., 2004. A method based on linguistic aggregation operators for group decision making with linguistic preference relations. Information sciences, 166(1-4), pp.19-30.

[52] Xu, Z., 2004. Uncertain linguistic aggregation operators based approach to multiple attribute group decision making under uncertain linguistic environment. Information sciences, 168(1-4), pp.171-184.

[53] Xu, Z., 2005, August. An approach based on similarity measure to multiple attribute decision making with trapezoid fuzzy linguistic variables. In International Conference on Fuzzy Systems and Knowledge Discovery (pp. 110-117). Springer, Berlin, Heidelberg.

[54] Xian, S., Dong, Y., Liu, Y. and Jing, N., 2018. A novel approach for linguistic group decision making based on generalized interval-valued intuitionistic fuzzy linguistic induced hybrid operator and TOPSIS. International Journal of Intelligent Systems, 33(2), pp.288-314.

[55] Ye, J., 2018. Multiple attribute decision-making method based on linguistic cubic variables. Journal of Intelligent \& Fuzzy Systems, 34(4), pp.2351-2361.

[56] Zadeh, L. A., 1965. Fuzzy sets. Information and control, 8(3), 338-353.

[57] Zadeh, L.A., 1975. The concept of a linguistic variable and its application to approximate reasoning-III. Information sciences, 9(1), pp.43-80.

[58] Zhang, H., 2014. Linguistic intuitionistic fuzzy sets and application in MAGDM. Journal of Applied Mathematics, 2014.

Department of Mathematics Abdul Wali Khan University Mardan, Pakistan

Email address: muhammadqiyas@awkum.edu.pk

Department of Mathematics Abdul Wali Khan University Mardan, Pakistan

Email address: saleemabdullah@awkum.edu.pk

Algebra and Applications Research Unit, Division of Computational Science, Faculty of Science, Prince of Songkla University, Hat Yai, Songkhla 90110, Thailand

Email address: ronnason.c@psu.ac.th

Department of Mathematics Abdul Wali Khan University Mardan, Pakistan

Email address: muneeza0315@gmail.com 


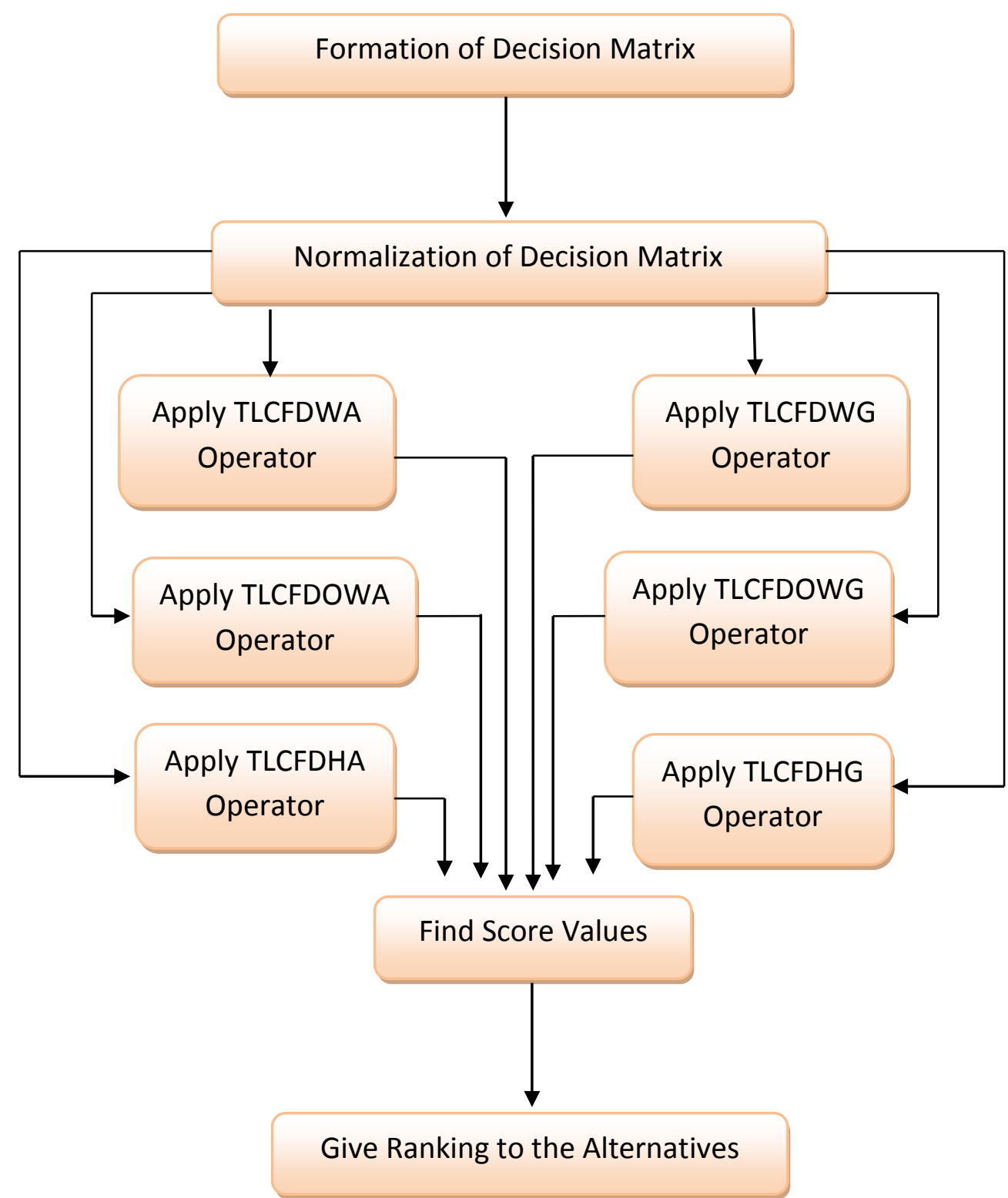

Figure 1. Flow chart of the proposed method 
Figure 2. Graph of the score values of Table 2.

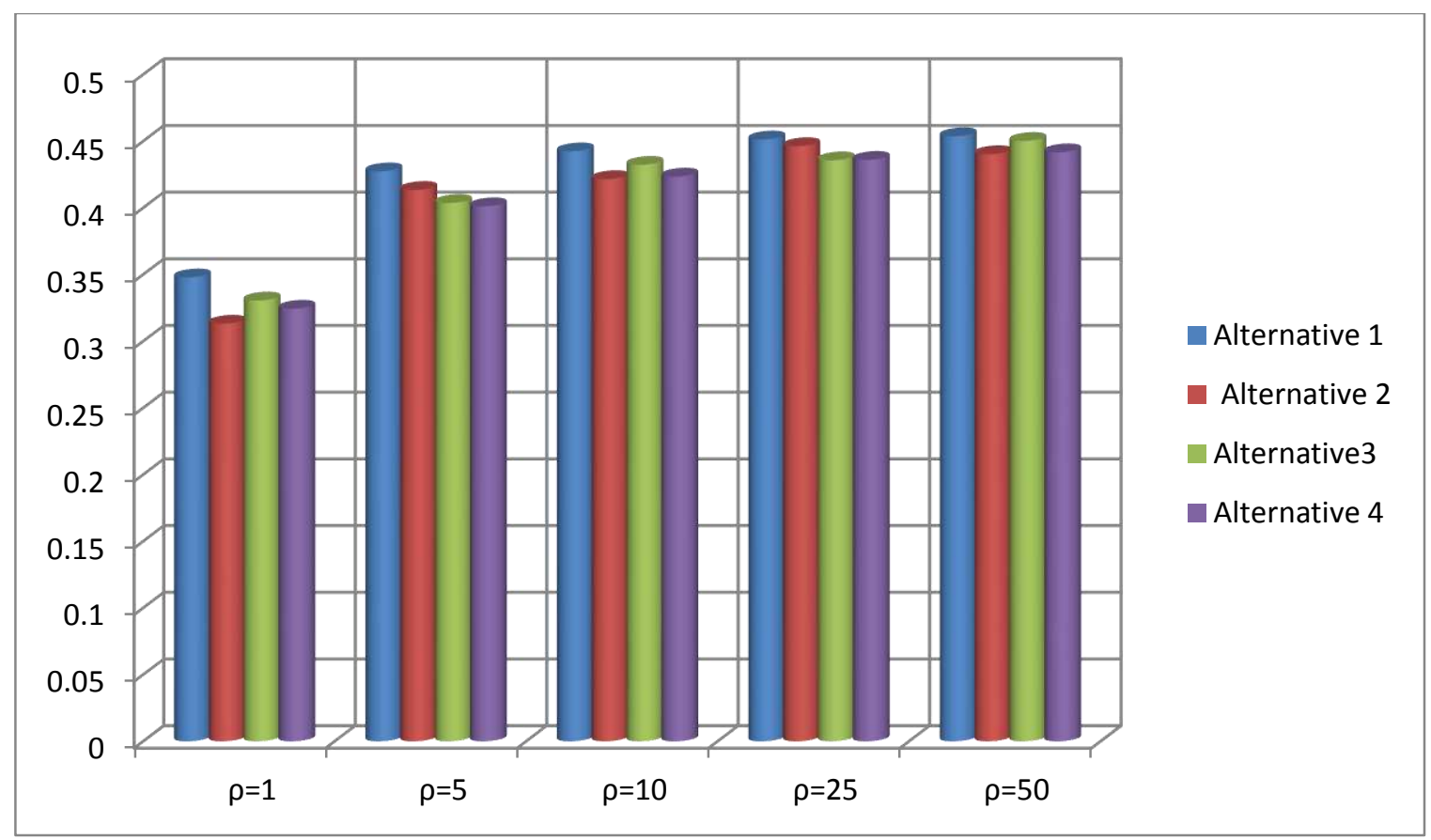


Figure 3. Graph of the score values of Table 3.

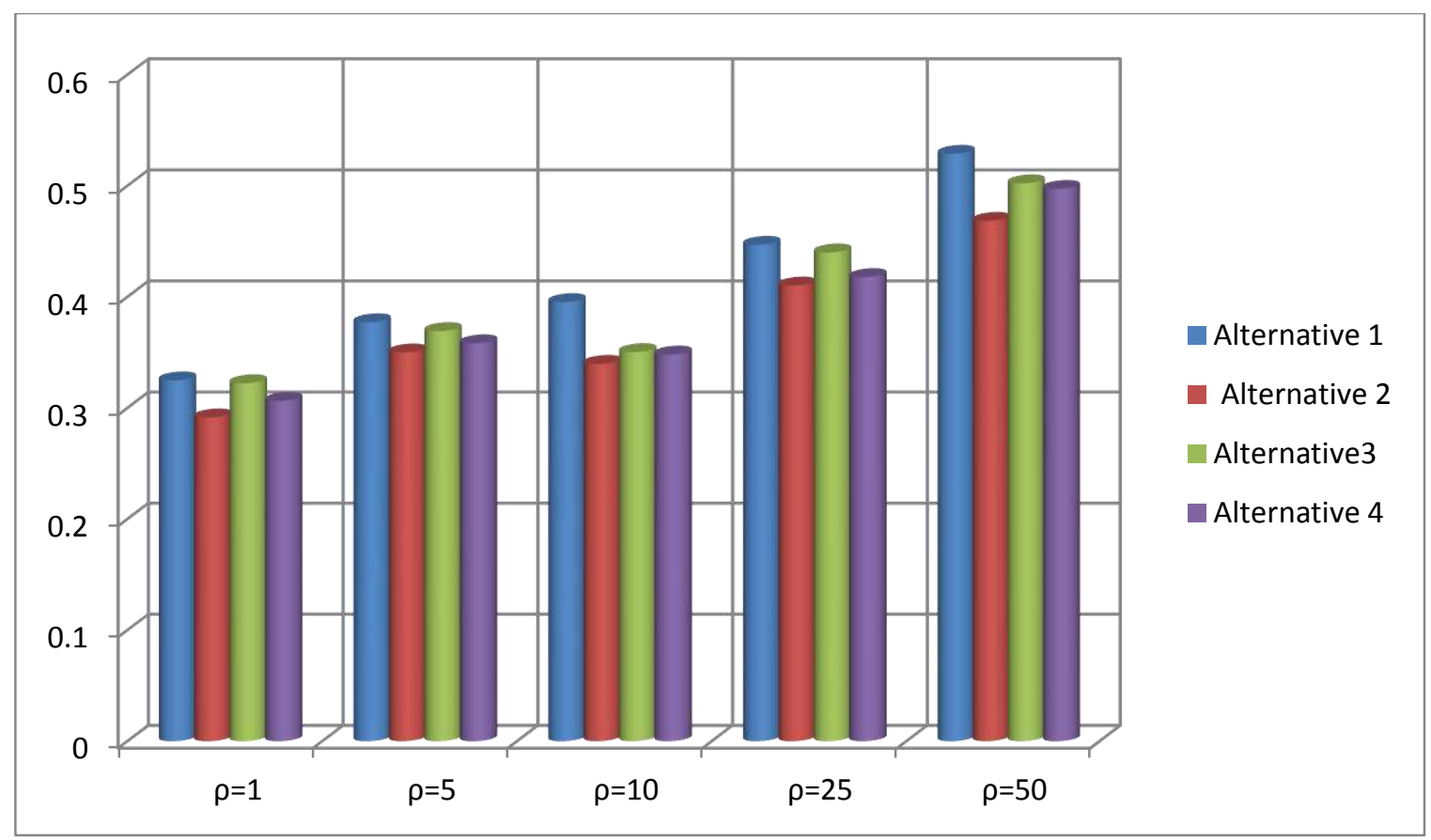


Figure 4. Graph of the score values of Table 4.

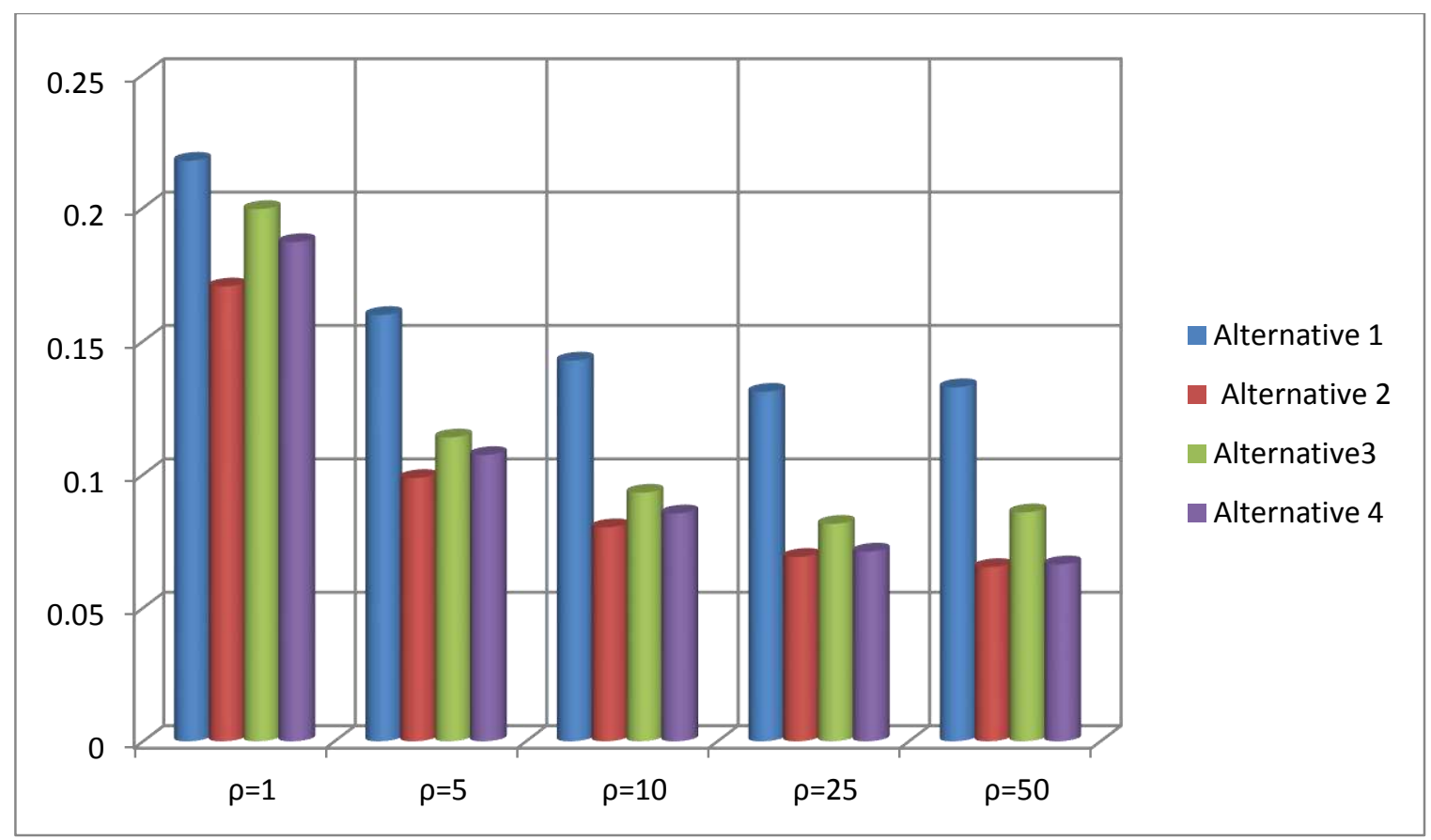


Figure 5. Graph of the score values of Table 5.

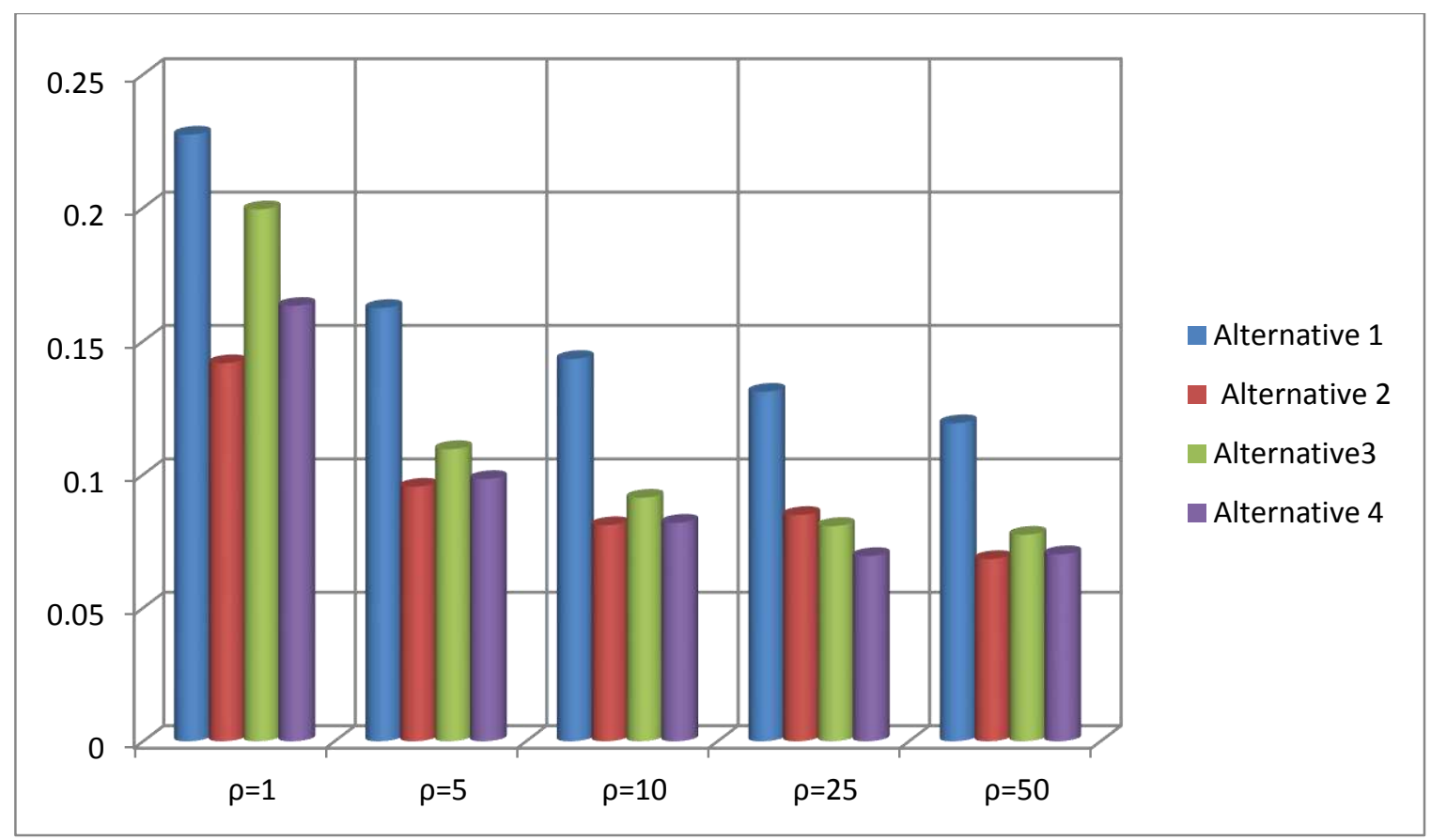


Figure 6. Graph of the score values of Table 6.

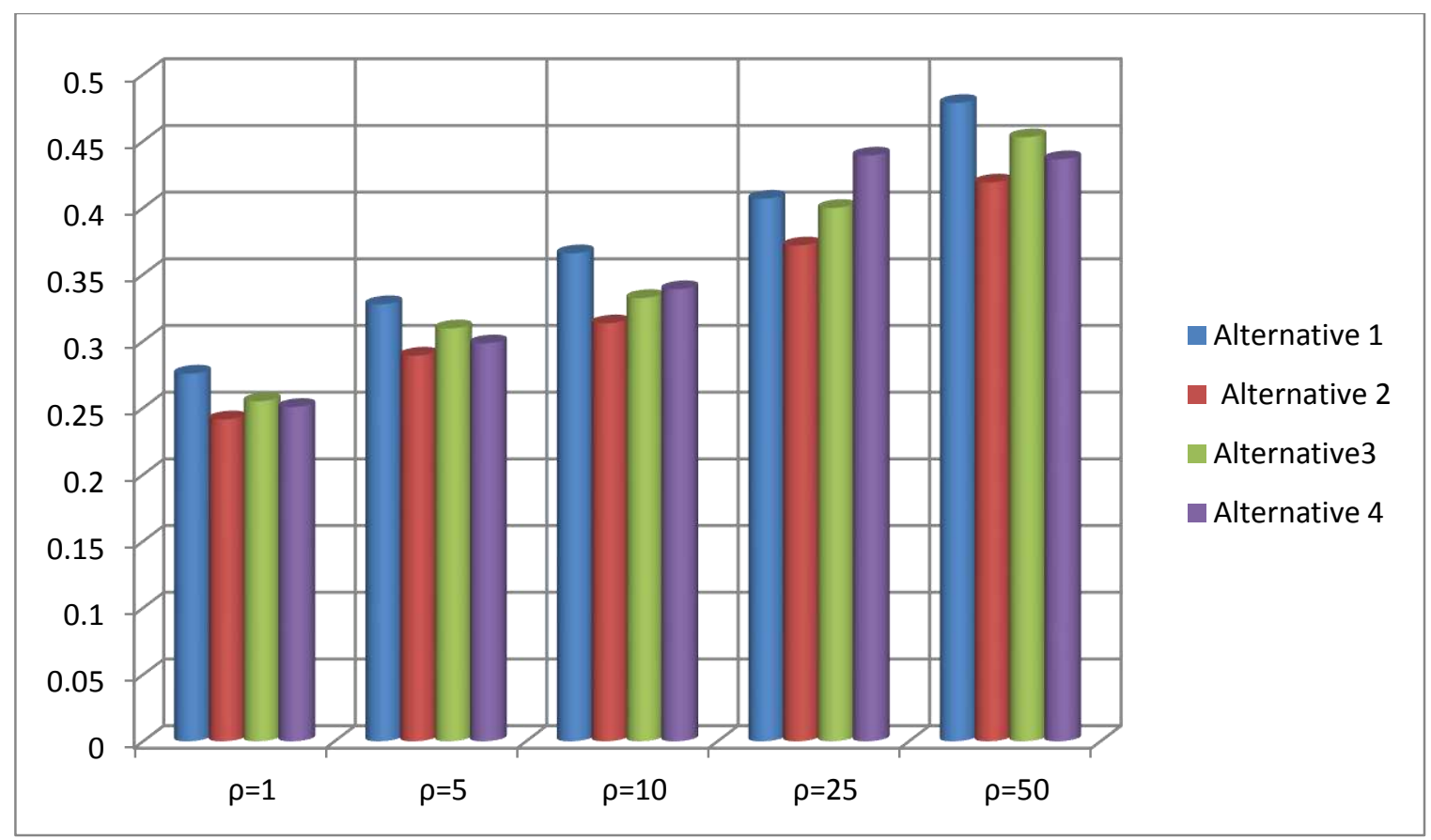


Figure 7. Graph of the score values of Table 7.

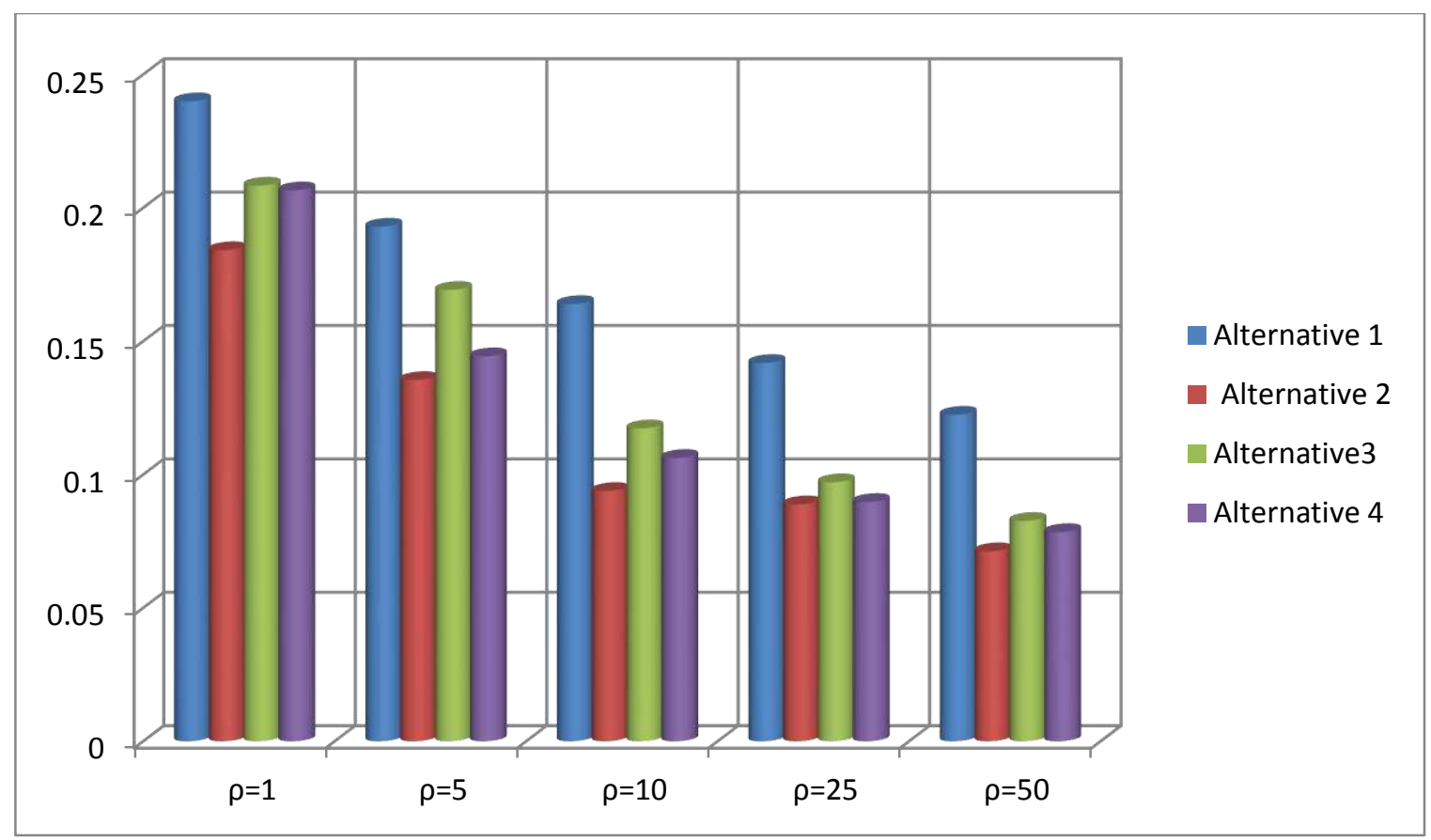

